\title{
SPECTRAL PROBLEMS IN OPEN QUANTUM CHAOS
}

\author{
STÉPHANE NONNENMACHER
}

\begin{abstract}
We present an overview of mathematical results and methods relevant for the spectral study of semiclassical Schrödinger (or wave) operators of scattering systems, in cases where the corresponding classical dynamics is chaotic; more precisely, we assume that in some energy range, the classical Hamiltonian flow admits a fractal set of trapped trajectories, which hosts a chaotic (hyperbolic) dynamics. The aim is then to connect the information on this trapped set, with the distribution of quantum resonances in the semiclassical limit.

Our study encompasses several models sharing these dynamical characteristics: free motion outside a union of convex hard obstacles, scattering by certain families of compactly supported potentials, geometric scattering on manifolds with (constant or variable) negative curvature. We also consider the toy model of open quantum maps, and sketch the construction of quantum monodromy operators associated with a Poincaré section for a scattering flow.

The semiclassical density of long living resonances exhibits a fractal Weyl law, related with the fact that the corresponding metastable states are "supported" by the fractal trapped set (and its outgoing tail). We also describe a classical condition for the presence of a gap in the resonance spectrum, equivalently a uniform lower bound on the quantum decay rates, and present a proof of this gap in a rather general situation, using quantum monodromy operators.
\end{abstract}

\section{Contents}

1. Introduction 2

1.1. Scattering vs. metastable states 4

1.2. Semiclassical distribution of resonances 5

1.3. Outline of the paper 8

2. Chaotic dynamics 9

2.1. Hyperbolicity 10

2.2. Complexity 11

3. Semiclassical formulation and more examples of chaotic flows 13

3.1. Potential scattering 13

3.2. Geometric scattering 15

4. "Massaging" $P(\hbar)$ into a proof of the fractal Weyl upper bound 17 
4.1. Complex scaling: resonances as spectrum of a nonselfadjoint operator 17

4.2. Conjugation by an escape function 19

5. Open quantum maps and quantum monodromy operators 20

5.1. Open quantum maps 20

5.2. Open quantum maps of finite rank 22

5.3. Poincaré sections 23

5.4. Quantum monodromy operators 24

6. From fractal Weyl upper bound to fractal Weyl law ? 26

6.1. Fractal Weyl law for open quantum maps 27

7. Interpretation of the fractal Weyl upper bound for open quantum maps 31

7.1. Heuristic explanations 31

7.2. A proof of the fractal Weyl upper bound 33

8. Resonance gap for open quantum maps and monodromy operators 35

8.1. Proof of the resonance gap in terms of the topological pressure 36

8.2. Is the pressure bound optimal? 39

9. Phase space structure of wavefunctions 44

9.1. Metastable states 44

9.2. Scattering states 47

10. Conclusion 49

Appendix A. A brief review of $\hbar$-pseudodifferential calculus 50

A.1. Weyl quantization and pseudodifferential calculus 50

A.2. Exotic symbol classes $\quad 52$

A.3. Fourier integral operators $\quad 52$

\begin{tabular}{ll} 
References & 53 \\
\hline
\end{tabular}

\section{INTRODUCTION}

This review article will present some recent results and methods in the study of 1particle quantum or wave scattering systems, in the semiclassical/high frequency limit, in cases where the corresponding classical/ray dynamics is chaotic.

The study of such systems has a long history in physics and mathematics, ranging from mesoscopic semiconductor physics to number theory. We will focus on some mathematical aspects, adopting a quantum chaos point of view: one wants to understand how the classical dynamics influences the quantum one, both regarding time dependent and time independent 
(that is, spectral) quantities. Equivalently, one searches for traces of classical chaos in the quantum mechanical system. In this introduction, I will focus on a simple system we will

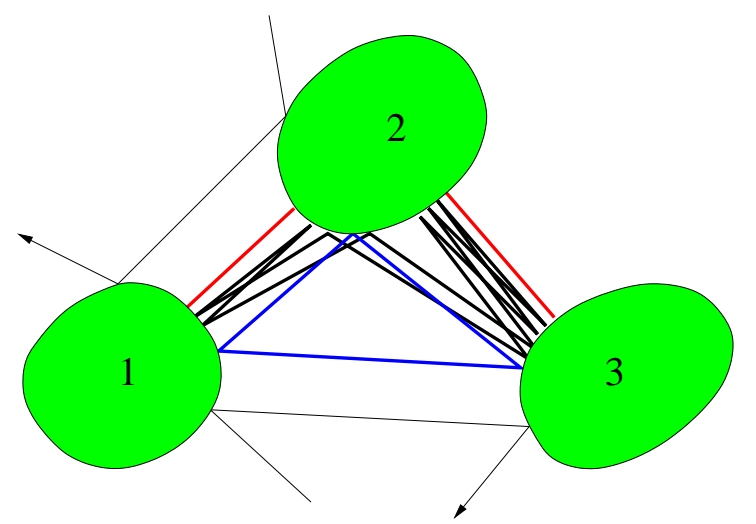

Figure 1. Configuration of 3 convex obstacles in the plane satisfying the noeclipse condition, leading to a fractal hyperbolic trapped set. The numbering of the obstacles leads to the associated symbolic dynamics.

be dealing with: the scattering by three or more balls $\left(B_{j}\right)_{j=1, \ldots, J}$ (more generally, strictly convex bodies with smooth boundaries) in $\mathbb{R}^{d}$, satisfying a no-eclipse condition [37] (see Fig. (1). The nature of the classical dynamics will be explained in $₫ 2$.

At the quantum level, one wants to understand the wave propagation in this geometry, that is solve the (scalar) wave equation

$$
\partial_{t}^{2} u(x, t)-\Delta_{\Omega} u(x, t)=0,
$$

with given initial conditions $u(x, 0), \partial_{t} u(x, 0)$. Here $\Delta_{\Omega}$ is the Laplacian outside the disks $\left(\Omega=\mathbb{R}^{d} \backslash \sqcup_{i} D_{i}\right)$, with Dirichlet boundary conditions. Through a Fourier transform in time, we get the Helmholtz equation

$$
\Delta_{\Omega} u(x)+k^{2} u(x)=0,
$$

which describes stationary waves of energy $k^{2}$ ( $k$ is the wavevector, that is the inverse of the wavelength).

If the particle propagating is a quantum one, its evolution rather satisfies the Schrödinger equation

$$
i \hbar \partial_{t} u(x, t)=-\frac{\hbar^{2} \Delta_{\Omega}}{2} u(x, t),
$$

where $\hbar$ is Planck's constant. We will see in \$3 that both equations (113) can be analyzed along the same lines in the high frequency/semiclassical limits.

\footnotetext{
${ }^{1}$ Namely, the convex hull of any pair of obstacles $B_{i}, B_{j}$ does not intersect any third obstacle.
} 
This scattering system is physically relevant, and has been studied in theoretical physics (see for instance the review paper by Wirzba [89] for the 2-dimensional scattering by $J$ disks, and references therein) and mathematics literature [37]. It has also been implemented in various experimental realizations, most recently on microwave tables by the Marburg group [87.

1.1. Scattering vs. metastable states. For a given wavevector $k$, Eq. (2) admits an infinite dimensional space of solutions $u(x)$, called scattering states, which can be parametrized by decomposing $u(x)$, away from the obstacles (say, outside a ball $B\left(0, R_{0}\right)$ ), into a basis of incoming and outgoing waves:

$$
u(x)=u_{\text {in }}(x)+u_{\text {out }}(x) .
$$

For instance, in dimension $d=2$ the ingoing waves can be expanded in angular momentum eigenstates: using polar coordinates $x=(r, \theta)$,

$$
u_{i n}(x)=\sum_{n \in \mathbb{Z}} a_{n}^{i n} e^{i n \theta} H_{n}^{i n}(k r),
$$

where $H_{n}^{i n}$ are the incoming Hankel functions, and similarly for $u_{\text {out }}(x)$. Any such solution $u(x)$ is called a scattering state. It is not $L^{2}$-normalizable, reflecting the fact that the spectrum of $-\Delta_{\Omega}$ is absolutely continuous on $\mathbb{R}_{+}$, without any embedded eigenvalue. We will briefly address the phase space structure of these scattering states in 99.2 .

Beyond the a.c. spectrum, this system admits a discrete set of quantum resonances, or complex generalized eigenvalues. They can be obtained as follows. The resolvent $\left(\Delta+k^{2}\right)^{-1}$ is a bounded operator on $L^{2}(\Omega)$ for $\operatorname{Im} k>0$; its norm diverges when $\operatorname{Im} k \rightarrow 0$, reflecting the presence of the continuous spectrum. However, if we cut it off by a compactly supported (or fast decaying) function $\chi(x)$, the cutoff resolvent $\chi\left(\Delta+k^{2}\right)^{-1} \chi$ can be meromorphically continued from the upper half plane $\{\operatorname{Im} k>0\}$ to the lower half plane $\{\operatorname{Im} k<0\}$, where it generally admits a discrete set of poles $\left\{k_{j}\right\}$ of finite multiplicities 2 . These poles are called the resonances of $-\Delta_{\Omega}$. Each pole $k_{j}$ (assuming it is simple) is associated with a generalized eigenfunction $u_{j}(x)$, which satisfies the equation

$$
\left(\Delta_{\Omega}+k_{j}^{2}\right) u_{j}(x)=0, \quad \text { with Dirichlet boundary conditions on } \partial \Omega,
$$

and is purely outgoing (meaning that its decomposition (4) only contains outgoing components). This function grows exponentially when $|x| \rightarrow \infty$, an "unphysical" behaviour, so it is only meaningful inside a compact set (the interaction region formed by the ball $\left.B\left(0, R_{0}\right)\right)$. The time dependent function

$$
\tilde{u}_{j}(x, t) \stackrel{\text { def }}{=} u_{j}(x) e^{-i k_{j} t}, \quad t \geq 0
$$

\footnotetext{
${ }^{2}$ In even dimension the continuation has a logarithmic singularity at $k=0$, often represented by a cut along the negative imaginary axis.
} 
satisfies Eq. (11). The time decay in (17) explains why $u_{j}(x)$ is called a metastable state, with lifetime

$$
\tau_{j}=\frac{1}{2\left|\operatorname{Im} k_{j}\right|} .
$$

One can expand the time dependent wave $u(x, t)$ when $t \rightarrow \infty$ into a sum over (at least some of) the metastable states (77). Such an expansion is less straightforward than in the case of a closed system (it isn't based on an $L^{2}$ orthogonal decomposition), but often gives a good description of the wave $u(x, t)$ for long times [84, 14, 32].

Another application of the study of resonances: the presence of a resonance free strip below the real axis (together with estimates of the resolvent in the strip) can be used to quantitatively estimate the dispersion and local energy decay for the wave $u(x, t)$, either in the case of the wave equation (1) or that of the (nonsemiclassical) Schrödinger equation $i \partial_{t} u=-\Delta_{\Omega} u[17,18,13]$.

1.2. Semiclassical distribution of resonances. We will not investigate these time dependent aspects any further, but will concentrate on the spectral one, namely the distribution of the resonances and the associated metastable states. The first mathematical works on the subject consisted in counting resonances in large disks $D(0, k), k \rightarrow \infty$. Melrose [48] obtained the general Weyl type upper bound $\mathcal{O}\left(k^{d}\right)$ for compact obstacles in odd dimension; this bound was generalized to obstacles in even dimension [86] as well as to scattering by a potential [91].

In the following we will focus on the long living resonances, that is those $k_{j}$ sitting within a fixed distance from the real axis (equivalently, the resonances with lifetimes $\tau_{j}$ uniformly bounded from below). These resonances are the most relevant ones for the long time behaviour of the waves. We will consider the high frequency limit $\operatorname{Re} k \gg 1$, which is equivalent with the semiclassical limit in quantum mechanics (see \$3), in order to establish a connection with the classical dynamics (see Fig,2).

\section{Questions:}

(1) For given width $W>0$ and depth $\gamma>0$, what is the asymptotic number of resonances in the rectangle $[k, k+W]-i[0, \gamma]$ when $k \rightarrow \infty$ ?

(2) In particular, is there some $\gamma>0$ such that this rectangle is empty of resonances for $k$ large enough? (such a $\gamma$ is called a resonance gap).

(3) Given an infinite sequence of long living resonances $\left(k_{j_{\ell}}\right)$, what is the spatial, or phase space structure of the associated metastable states when Re $k_{j_{\ell}} \rightarrow \infty$ ?

In this high frequency limit, these spectral questions will be connected with long time properties of the classical dynamics of the system. This dynamics consists in following straight rays at unit speed outside the obstacles, and reflecting specularly on the obstacles. In mathematical notations, this dynamics is a flow $\Phi^{t}$ defined on the phase space formed 


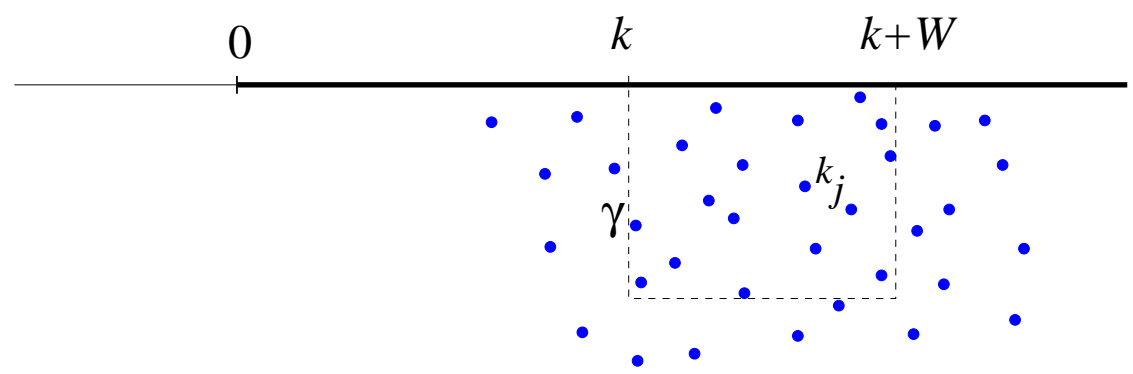

Figure 2. Absolutely continuous spectrum of $\Delta_{\Omega}$, together with the resonances below the real axis, near some value $k \gg 1$.

by the unit cotangent bundle

$$
S^{*} \Omega=\left\{(x, \xi), x \in \Omega, \xi \in \mathbb{R}^{d},|\xi|=1\right\},
$$

where the speed $\xi$ is equal to the momentum. For each time $t \in \mathbb{R}$, the flow $\Phi^{t}$ maps any initial phase space point $(x, \xi)$ in to its position $\Phi^{t}(x, \xi)$ at time $t$.

With our conditions on the obstacles, this dynamics is chaotic in the following sense: the set of trapped trajectories

$$
K=\left\{\rho \in S^{*} \Omega, \quad \Phi^{t}(\rho) \text { uniformly bounded when } t \rightarrow \pm \infty\right\}
$$

is a fractal flow-invariant set, and the flow on it is uniformly hyperbolic (equivalently, one says that $K$ is a hyperbolic set for $\Phi^{t}$, see $\oint 2$ for details). For future use we also define the outgoing $\left(K^{+}\right)$and incoming $\left(K^{-}\right)$tails of the trapped set,

$$
K^{ \pm}=\left\{\rho \in S^{*} \Omega, \quad \Phi^{t}(\rho) \text { uniformly bounded when } t \rightarrow \mp \infty\right\},
$$

with the obvious property $K=K^{-} \cap K^{+}$.

The question (2) above has been addressed around the same time by Ikawa [37] and Gaspard and Rice [28] (see also [12]). Both these works establish the presence of a gap, provided the trapped set $K$ is sufficiently "filamentary". The precise criterion depends on a certain dynamical quantity associated with the flow, a topological pressure defined in terms of the unstable Jacobian $\varphi^{+}(\rho)$ which measures the instability of the trajectories (these

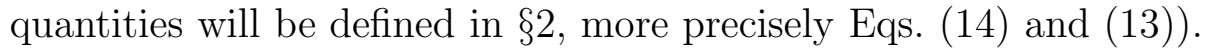

Theorem 1. 37] Consider the obstacle scattering problem in $\mathbb{R}^{d}$, with strictly convex obstacles satisfying the no-eclipse condition. If the topological pressure for the flow on the trapped set $K \subset S^{*} \Omega$ satisfies

$$
\mathcal{P}=\mathcal{P}\left(-\varphi^{+} / 2, \Phi^{t} \uparrow_{K}\right)<0
$$

then for any small $\epsilon>0$ there exists $k_{\epsilon}>0$ such that $\Delta_{\Omega}$ has no resonance in the strip

$$
\left[k_{\epsilon}, \infty\right)-i[0,|\mathcal{P}|-\epsilon] .
$$


In dimension $d=2$, the sign of the above topological pressure is determined by purely geometric data, namely the Hausdorff dimension of the trapped set:

$$
\mathcal{P}\left(-\varphi^{+} / 2, \Phi^{t} \uparrow_{K}\right)<0 \Longleftrightarrow \operatorname{dim}_{H}(K)<2,
$$

which gives a precise notion of "filamentary" or "thin" trapped set (notice that $K$ is embedded in the 3 -dimensional phase space $S^{*} \Omega$ ).

In 88.1 we will sketch the proof of the above theorem (in a more general context), using the tool of quantum monodromy operators. The intuitive idea is the following: wavepackets propagating along $K$ disperse exponentially fast due to the hyperbolicity of the trajectories; on the other hand, the wavepackets propagating on nearby trajectories could also interfere constructively in order to recombine themselves along $K$. The pressure criterion ensures that the dispersion is stronger than the possible constructive interference, leading to a global decay of the wave near $K$.

The pressure $\mathcal{P}\left(-\varphi^{+} / 2\right)$ will appear several times in the text. Its value somehow determines a dichotomy between the "very open" scattering systems with "thin" trapped sets $\left(\mathcal{P}\left(-\varphi^{+} / 2\right)<0\right)$, and the "weakly open" ones with "thick" trapped sets $\left(\mathcal{P}\left(-\varphi^{+} / 2\right) \geq 0\right)$. It will be relevant also in the description of the scattering states in $\$ 9.2$.

The question (1) has first been addressed by Sjöstrand in the case of a real analytic Hamiltonian flow with a chaotic trapped set [74], leading to the first example of fractal Weyl upper bound. His result was generalized and sharpened in 78, see Thm 4 below. For the above obstacle scattering, a similar fractal upper bound had been conjectured in [74], but proved only recently [54].

To state the result, we recall the definition of the upper box (or Minkowski) dimension of a bounded set $K \subset \mathbb{R}^{n}$ :

$$
\overline{\operatorname{dim}}(K) \stackrel{\text { def }}{=} \limsup _{\epsilon \rightarrow 0}\left(n-\frac{\log \operatorname{Vol}\left(K_{\epsilon}\right)}{\log \epsilon}\right)
$$

where $K_{\epsilon}$ is the $\epsilon$-neighbourhood of $K$. The dimension is said to be pure if $\frac{\operatorname{Vol}\left(K_{\epsilon}\right)}{\epsilon^{n-\overline{\operatorname{dim}}(K)}}$ is uniformly bounded as $\epsilon \rightarrow 0$.

Theorem 2. [54] Consider the obstacle scattering problem in $\mathbb{R}^{d}$, with strictly convex obstacles satisfying the no-eclipse condition.

Let $2 \nu+1$ be the upper box dimension of the trapped set $K \subset S^{*} \Omega$.

Then, the resonances of $\Delta_{\Omega}$ satisfy the following bound. For any $\gamma>0$ and any $\epsilon>0$, there exists $k_{\gamma, \epsilon}, C_{\gamma, \epsilon}>0$ such that

$$
\forall k>k_{\gamma, \epsilon}, \quad \sharp\left\{k_{j} \in[k, k+1]-i[0, \gamma]\right\} \leq C_{\gamma, \epsilon} k^{\nu+\epsilon} .
$$

If $K$ is of pure dimension $2 \nu+1$, one can take $\epsilon=0$.

In dimension $d=2$, the trapped set is always of pure dimension, and its box dimension is equal to its Hausdorff dimension. In that case, the dimension $\nu$ can be obtained through 
the topological pressure of the flow on $K$ (see \$2.2), namely $\nu$ is the (unique) real root $s_{0}$ of the equation

$$
\mathcal{P}\left(-s \varphi^{+}, \Phi^{t} \uparrow K\right)=0
$$

(because $\mathcal{P}\left(-s \varphi^{+}\right)$is strictly decreasing with $s$, this equation directly leads to the equivalence (11)).

The question (3) has been studied only recently, in the case of a smooth Hamiltonian flow, or a discrete time dynamics (open map). The main phenomenon is that, in the high frequency limit, the long living metastable states are microlocalized near the outgoing tail $K^{+}$, and can be described in terms of semiclassical measures which are invariant through the flow, up to a global decay (see \$9.1). Although no rigorous result on this question has been obtained in the case of obstacles, it is very likely that Thm. 10 can be adapted to the obstacles setting.

1.3. Outline of the paper. In the next section we describe the dynamical properties of the classical flows we wish to consider, namely Hamiltonian flows for which the trapped set is a compact hyperbolic repeller. We also define the relevant dynamical quantities associated with the flow, like the unstable Jacobian and the topological pressure.

In $\S 3$ we extend the above two theorems to more general systems, namely semiclassical Schrödinger operators $P(\hbar)$ involving a compactly supported potential, and LaplaceBeltrami operators on Riemannian manifolds, where the dynamics is only driven by the geometry. We state the analogues of Thms. 1 1 and 2 in these settings. The case of hyperbolic manifolds of infinite volume (obtained as quotients of the Poincaré half-space $\mathbb{H}^{d}$ by certain discrete groups) is particularly interesting: the quantum resonances of the Laplacian can then be directly connected with the classical dynamics.

In $\$ 4$ we interpret the quantum resonances as the eigenvalues of a (nonselfadjoint) operator obtained by "deforming" $P(\hbar)$ into the complex plane. We then (sketchily) explain how a further deformation, using microlocal weights, allows to prove a fractal Weyl upper bound for the number of resonances 78 .

In $\$ 5$ we introduce the model of open (chaotic) maps and their quantizations, which correspond to discrete time dynamics instead of flows. They have been used as a convenient toy model for the "true" scattering systems, being much more amenable to numerical studies. We then construct quantum monodromy operators associated with a scattering Hamiltonian $P(\hbar)$; they form a family of open quantum maps associated with the Poincaré map for the classical flow, and can be used to characterize and study the resonances of $P(\hbar)$.

In $\$ 6$ we formulate a weak and a strong form of fractal Weyl law, and discuss their validity for the various systems introduced above, mostly guided by numerical data. In 97 we give a heuristic explanation of the Weyl law for quantum maps, and provide a proof of its upper bound, eventually leading to Thm 2 and its analogues. The proof shows how the full quantum system can be reduced to an effective operator of minimal rank. 
In $\S 8$ we show that the pressure criterion of Thm 1 applies to all the systems considered above. We sketch the proof of this gap in the case of open quantum maps and monodromy operators, leading to the general case of Schrödinger operators. In $\$ 8.2$ we discuss the sharpness of this criterion, using both analytical and numerical results.

In $₫ 9$ we briefly describe what is known about the phase space structure of metastable states associated with the long living resonances, in particular using the tool of semiclassical measures. We also consider the scattering states.

Finally, $\$ 10$ presents a brief conclusion, and mentions possible extensions of the methods to similar nonselfadjoint spectral problems, like the case of damped waves on a compact manifold of negative curvature.

Most of the above results have appeared elsewhere (or are bound to do so in a near future). The spectral radius estimate for open quantum maps, Thm 9, has not been formulated before, but it is a rather direct application of [57]. The numerics of $₫ 8.2 .2$ had not been published either.

Acknowledgements. I have benefitted from many interesting discussions on this topic, notably with M.Zworski, C.Guillarmou, F.Naud, M.Novaes, M.Sieber and J.Keating. I thank C.Guillarmou and F.Naud for communicating to me their recent results on scattering states. I am also grateful to M.Zworski, M.Novaes and J.Keating for their permission to use some figures from earlier publications. I have been partially supported by the Agence National de la Recherche through the grant ANR-09-JCJC-0099-01. Finally, I thank both anonymous referees for their careful reading and constructive comments.

\section{Chaotic Dynamics}

We have already introduced the flow $\Phi^{t}$ on the phase space $S^{*} \Omega$ describing the classical scattering system outside the obstacles: it consists in the free motion at unit speed outside the obstacles, plus specular reflection at the boundaries. This flow is generated by the Hamiltonian vector field $H_{p}=\frac{\partial p}{\partial \xi} \partial_{x}-\frac{\partial p}{\partial x} \partial_{\xi}$ associated with the Hamilton function

$$
p(x, \xi)=\frac{|\xi|^{2}}{2}+V_{\Omega}(x), \quad \text { with the singular potential } \begin{cases}V_{\Omega}(x)=0, & x \in \Omega, \\ V_{\Omega}(x)=\infty, & \text { otherwise }\end{cases}
$$

The unit cotangent bundle $S^{*} \Omega$ is equivalent with the energy shell $p^{-1}(1 / 2)$.

All the flows we will consider are Hamiltonian, in particular they preserve the natural symplectic form on $T^{*} \mathbb{R}^{d}$. The discrete time models (open maps) introduced in $\$ 5$ will be given by local diffeomorphisms on a symplectic manifold, which also preserve the symplectic structure. All these systems are therefore conservative.

We will also make strong dynamical assumptions on these flows (or open maps), namely an assumption of strong chaos. The chaotic properties refer to the long time properties of the flow restricted to the trapped set $K$ (below we keep the notations of the obstacle 


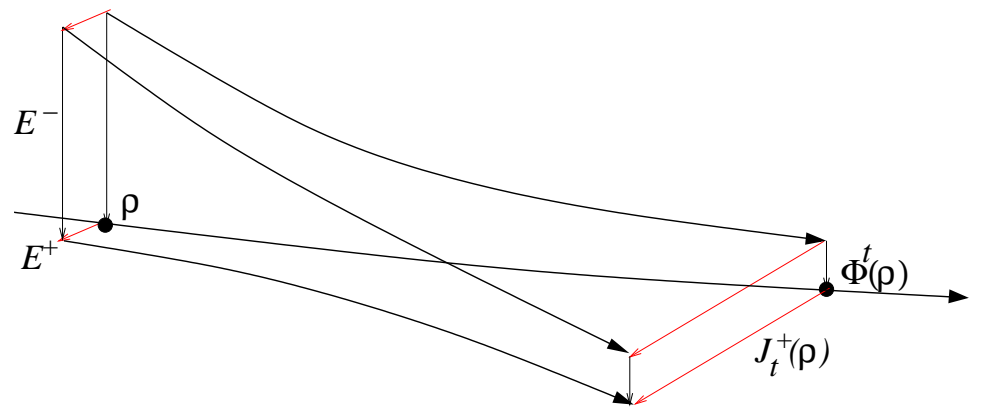

FiguRE 3. Hyperbolicity of the trajectory $\Phi^{t}(\rho)$ : nearby orbits along the stable (resp. unstable) directions approach $\Phi^{t}(\rho)$ exponentially fast in the future (resp. past). The unstable Jacobian $J_{t}^{+}(\rho)$ measures this exponential divergence.

problem, while the concepts apply as well to more general Hamiltonian flows or to maps). In our setting, chaos is the mixture of two components, namely hyperbolicity and complexity (see e.g. the textbooks [41, 11]).

2.1. Hyperbolicity. Firstly, the trapped set $K$ is assumed to be compact, and the flow $\Phi^{t} \uparrow K$ is assumed to be uniformly hyperbolic (see Fig. 3). This means that there is no fixed point $\left(H_{p} \neq 0\right)$, and for any $\rho \in K$, the tangent space $T_{\rho}\left(S^{*} \Omega\right)$ splits into the flow direction $\mathbb{R} H_{p}(\rho)$, a stable subspace $E^{-}(\rho)$, and an unstable subspace $E^{+}(\rho)$ :

$$
T_{\rho}\left(S^{*} \Omega\right)=\mathbb{R} H_{p}(\rho) \oplus E^{-}(\rho) \oplus E^{+}(\rho) .
$$

The (un)stable subspaces are characterized as follows: there exist $C, \lambda>0$ such that, for any $\rho \in K$,

$$
v \in E^{\mp}(\rho) \Longleftrightarrow \forall t>0, \quad\left\|d \Phi^{ \pm t} v\right\| \leq C e^{-\lambda t}\|v\| .
$$

The subspaces $E^{ \pm}(\rho)$ depend continuously on $\rho \in K$, and are uniformly transverse to each other. An important quantity is the unstable Jacobian of the flow,

$$
J_{t}^{+}(\rho) \stackrel{\text { def }}{=} \operatorname{det}\left(d \Phi^{t} \uparrow_{E^{+}(\rho)}\right), \quad t>0
$$

which measures the expansion of the flow along the unstable manifold $\sqrt{3}$. This Jacobian grows exponentially when $t \rightarrow \infty$. The infinitesimal version of this Jacobian reads

$$
\varphi^{+}(\rho) \stackrel{\text { def }}{=} \frac{d J_{t}^{+}}{d t}(\rho) \Upsilon_{t=0}
$$

and it is possible to choose a metric near $K$ such that $\varphi^{+}$is positive on $K$.

\footnotetext{
${ }^{3}$ Although this Jacobian depends on the choice of coordinates and metric near $\rho$ and $\Phi^{t}(\rho)$, its asymptotical behaviour for $t \rightarrow \infty$ does not.
} 
The (un)stable subspaces have nonlinear counterparts, namely the (un)stable manifolds

$$
W^{\mp}(\rho)=\left\{\rho^{\prime} \in S^{*} \Omega, \operatorname{dist}\left(\Phi^{t}\left(\rho^{\prime}\right), \Phi^{t}(\rho)\right) \stackrel{t \rightarrow \pm \infty}{\longrightarrow} 0\right\} .
$$

The unions of these manifolds makes up the incoming/outgoing tails $K^{\mp}(9)$.

A trapped set $K$ hosting such dynamical properties is called a hyperbolic set, or hyperbolic repeller.

In case of the scattering by $J \geq 2$ convex obstacles, the hyperbolicity is due to the strict convexity (or positive curvature) of the obstacles, which defocusses incoming parallel rays at each bounce. On the opposite, in the geometric scattering models of $\$ 3.2$, the defocussing is due to the negative curvature of the manifold.

2.2. Complexity. The second ingredient of a chaotic flow is complexity, in the information theoretic sense. It means that the trapped set $K$ cannot be too simple, e.g it cannot just consist in finitely many periodic trajectories. Grossly speaking, complexity means that, if one groups the long segments of trajectories into "pencils" of nearby segments, then the number of such pencils grows exponentially with the length of the segments.

Let us make this notion more explicit for our obstacle problem (see Fig. 1). To any point $\rho \in K$ away from the obstacle, we can associate a bi-infinite sequence of symbols

$$
\boldsymbol{\epsilon}=\cdots \epsilon_{-2} \epsilon_{-1} \cdot \epsilon_{0} \epsilon_{1} \epsilon_{2} \cdots, \quad \epsilon_{i} \in\{1,2, \cdots, J\}
$$

indexing the obstacles successively hit by $\Phi^{t}(\rho)$ in the future or in the past. This sequence obviously satisfies the condition $\epsilon_{i} \neq \epsilon_{i+1}$ for all $i \in \mathbb{Z}$.

Conversely, the assumptions we put on the obstacles imply that, for any sequence satisfying the above condition, one can construct a trajectory with the above properties, and this trajectory is (essentially) unique. In particular, this trajectory is periodic iff the sequence $\boldsymbol{\epsilon}$ is so. This description of the trapped orbits in terms of sequences of "symbols" is called a symbolic dynamics. It is a simple way to classify the trajectories of the flow, and estimate its complexity. For instance, if one decides to group trajectories by specifying the obstacles they hit from $i=0$ to $i=n$, then the number of such "pencils" is $J(J-1)^{n}$, which obviously grows exponentially with the "discrete time" $n$. The number of periodic orbits also grows exponentially w.r.t. their periods.

This complexity can be made quantitative through the topological pressures $\mathcal{P}\left(f, \Phi^{t} \uparrow K\right)$ associated with the flow on $K$ and "observables" $f \in C(K)$. The pressures provide a statistical information on the "pencils" of long orbit segments. In the present case of a hyperbolic repeller, this pressure can be defined in terms of long periodic orbits:

$$
\mathcal{P}\left(f, \Phi^{t} \uparrow_{K}\right) \stackrel{\text { def }}{=} \lim _{T \rightarrow \infty} \frac{1}{T} \log \sum_{p: T-1 \leq T_{p} \leq T} \exp (f(p)), \quad f(p)=\int_{0}^{T_{p}} f\left(\Phi^{t}\left(\rho_{p}\right)\right) d t .
$$

The sum runs over all the periodic orbits $p$ of periods $T_{p} \in[T-1, T]$, and $\rho_{p}$ is any point on $p$. In $₫ 9$ we will give an alternative way to compute the topological pressure for the open 
baker's map, in terms of symbolic dynamics. The pressure can also be defined through a variational formula over the probability measures $\mu$ on $K$ which are invariant by the flow:

$$
\mathcal{P}\left(f, \Phi^{t} \uparrow_{K}\right)=\sup _{\mu}\left\{H_{K S}(\mu)+\int f d \mu\right\}
$$

where $H_{K S}(\mu)$ is the Kolmogorov-Sinai entropy of the measure $\mu$ (with respect to the flow), a nonnegative number quantifying the "complexity of a $\mu$-typical trajectory".

If one takes $f \equiv 0$, the above expression measures the exponential growth rate of the number of long periodic orbits, which defines the topological entropy of the flow:

$$
\mathcal{P}\left(0, \Phi^{t} \uparrow_{K}\right)=H_{t o p}\left(\Phi^{t} \uparrow_{K}\right)=\sup _{\mu} H_{K S}(\mu) \text {. }
$$

For this reason, complexity is often defined by the positivity of $H_{t o p}$.

If $f$ is negative everywhere, the sum in (14) shows a competition between exponentially decreasing terms $e^{f(p)} \sim e^{T \bar{f}}$, and the exponentially increasing number of terms. This is the case, for instance, if one uses the unstable Jacobian (13) and takes $f=-s \varphi^{+}$for some parameter $s>0$. In that case $e^{f(p)}=J_{T_{p}}^{+}\left(\rho_{p}\right)^{-s}$ measures the instability of the orbit $p$. When $s=1$, the exponential damping exceeds the exponential proliferation, and the pressure is negative. Actually,

$$
\gamma_{c l} \stackrel{\text { def }}{=}-\mathcal{P}\left(-\varphi^{+}, \Phi^{t} \uparrow_{K}\right)>0
$$

defines the classical decay rate of the flow, which has the following physical meaning. Consider an initial smooth probability measure $\mu_{0}=g_{0}(\rho) d \rho$ on $S^{*} \Omega$, with the density $g_{0}$ supported inside the interaction region $S^{*} B\left(0, R_{0}\right)$, with $g_{0}(\rho)>0$ at some point $\rho \in K^{-}$. If we push forward this measure through $\Phi^{t}$, the mass of the interaction region will asymptotically decay as

$$
\left[\left(\Phi^{t}\right)^{*}\right] \mu_{0}\left(S^{*} B\left(0, R_{0}\right)\right) \sim C e^{-t \gamma_{c l}}, \quad t \rightarrow \infty .
$$

Below we will mostly be interested by the pressure with observable $f=-\varphi^{+} / 2$. It can be compared with the two quantities defined above. Indeed, using the variational formula (15) for the pressure, we easily get

$$
-\gamma_{c l} / 2 \leq \mathcal{P}\left(-\varphi^{+} / 2\right) \leq \frac{1}{2}\left(H_{t o p}-\gamma_{c l}\right) .
$$

The upper bound embodies the fact that $\mathcal{P}\left(-\varphi^{+} / 2\right)$ is negative if the dynamics on $K$ is "more unstable than complex".

In dimension $d=2$ the Hausdorff dimension of $K$ can be obtained in terms of the topological pressure: $\operatorname{dim}_{H}(K)=2 s_{0}+1$, where $s_{0} \in[0,1]$ is the unique root of the equation $\mathcal{P}\left(-s \varphi^{+}\right)=0$. In particular, one gets the equivalence (11). Hence, hyperbolicity and complexity directly influence the (fractal) geometry of the trapped set.

In $\$ 5$ we introduce open maps $\kappa$, which are local diffeomorphisms defined on some open subset of a symplectic manifold. The definition of the trapped set, and of hyperbolicity, 
are very similar with the case of flows. Since the Jacobian $J_{t}^{+}$only makes sense for integer times, we take $\varphi^{+}=\log J_{1}^{+}$. The topological pressure can be defined as in (14), with $f(p)$ given by a sum over the points in $p$.

\section{SemiclassicAl FORMUlation AND MORE EXAMPLES OF CHAOTIC FLOWS}

In the high frequency limit $(k \gg 1)$, the Helmholtz equation (2) can be rewritten using an small positive parameter, which we call $\hbar$ by analogy with Planck's constant. This parameter scales as

$$
\frac{1}{C} \leq \hbar k \leq C \quad\left(\text { in short }, \hbar \asymp k^{-1}\right),
$$

so the high-frequency limit is equivalent with the semiclassical limit $\hbar \rightarrow 0$. The equation (2) now takes the form of a time-independent Schrödinger equation:

$$
-\frac{\hbar^{2} \Delta_{\Omega}}{2} u=E(\hbar) u, \quad \text { with energy } \quad E(\hbar)=\hbar^{2} k^{2} / 2 \in\left[\frac{1}{2 C^{2}}, \frac{C^{2}}{2}\right] .
$$

Here the operator (quantum Hamiltonian)

$$
P(\hbar)=-\frac{\hbar^{2} \Delta_{\Omega}}{2}
$$

is the quantization of the classical Hamiltonian (12).

Remark 3.1. The operator $P(\hbar)$ is also the generator of the semiclassical Schrödinger equation (3) which describes the scattering of a quantum (scalar) particle, $u(x, t)$ being the wavefunction of the particle at time $t$. The resonant states $u_{j}(x)$ of ([6) satisfy the equation $P(\hbar) u_{j}=z_{j}(\hbar) u_{j}$, with the correspondence

$$
z_{j}(\hbar)=\hbar^{2} k_{j}^{2} / 2 .
$$

According to the Schrödinger equation (3) , these states decay with a rate $\left|\operatorname{Im} z_{j}(\hbar)\right| / \hbar$, which is not the same as the decay rate $\left|\operatorname{Im} k_{j}\right|$ associated with the wave equation (1). Yet, if we consider resonances in a "semiclassical box" $\left\{\operatorname{Re}\left(\hbar k_{j}\right) \in[1 / C, C], \operatorname{Im} k_{j} \in[-C, 0]\right\}$ for some fixed $C>1$, then two decay rates are comparable: $\operatorname{Im} z_{j}(\hbar) / \hbar=\operatorname{Re}\left(\hbar k_{j}\right) \operatorname{Im} k_{j}+\mathcal{O}(\hbar)$.

3.1. Potential scattering. The introduction of $\hbar$ in the obstacle problem is merely a convenient bookkeeping parameter in the high frequency limit. More importantly, it allows to extend the our study to more general scattering Hamiltonian flows, typically by replacing the obstacle potential $V_{\Omega}$ by a smooth potential $V \in C_{c}^{\infty}\left(\mathbb{R}^{d}\right)$, leading to the classical Hamiltonian

$$
p(x, \xi)=\frac{|\xi|^{2}}{2}+V(x), \quad(x, \xi) \in T^{*} \mathbb{R}^{d}
$$

which generates a smooth flow $\Phi^{t}$ on the phase space $T^{*} \mathbb{R}^{d}$. 

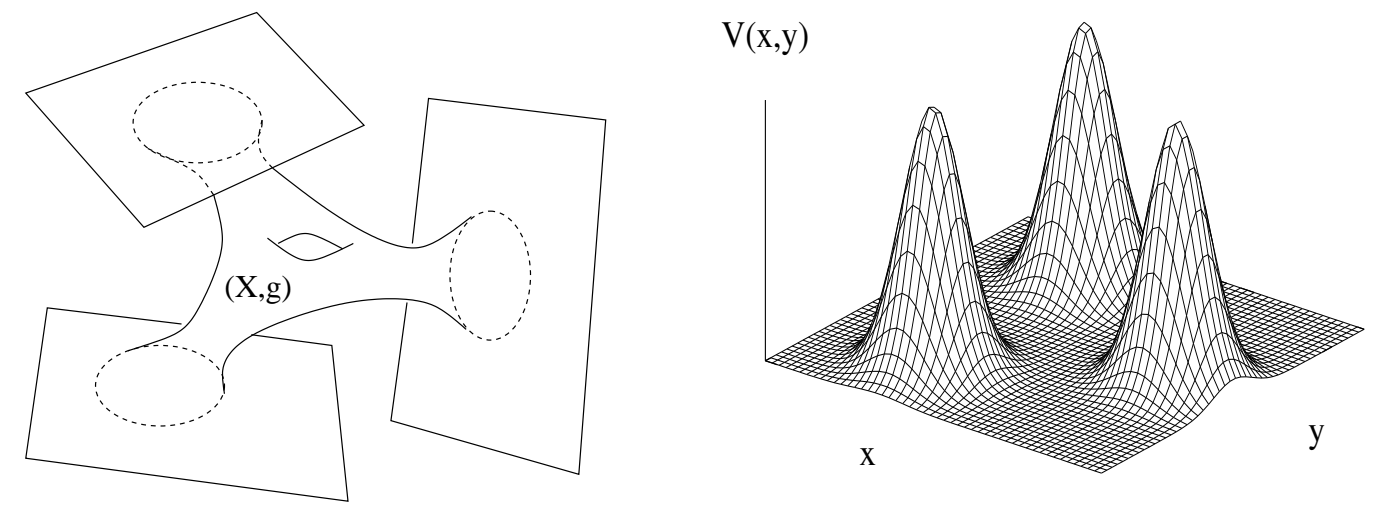

FiguRE 4. Left: a Riemannian surface with 3 Euclidean ends. Right: a potential $V \in C_{c}^{\infty}\left(\mathbb{R}^{2}\right)$, with Hamiltonian flow hyperbolic on the trapped set $K_{E}$ in a range of energies.

The $\hbar$-quantization of this Hamiltonian (see Appendix $\mathrm{A}$ ) is the Schrödinger operator

$$
P(\hbar)=-\frac{\hbar^{2} \Delta}{2}+V(x)
$$

where $\Delta$ is the Laplacian on $\mathbb{R}^{d}$. We say that $p(x, \xi)$ is the semiclassical symbol of the operator $P(\hbar)$ (see the Appendix for a short reminder on $\hbar$-quantization). If $\operatorname{supp} V$ is contained in a ball $B\left(0, R_{0}\right)$, we will call this ball the interaction region.

As opposed to the obstacle case, the flow on the energy shell $p^{-1}(E), E>0$, is not obtained through a simple rescaling of the flow at energy $1 / 2$ : both dynamics can be drastically different. Similarly, at the quantum level, $P(\hbar)$ depends on $\hbar$ in a nontrivial way. It is easy to produce a smooth potential $V(x)$ such that the flow on the energy shell $p^{-1}(E)$ is chaotic in some range $\left[E_{1}, E_{2}\right]$, in the sense that for any energy $E \in\left[E_{1}, E_{2}\right]$ the trapped set

$$
K_{E}=\left\{\rho \in p^{-1}(E), \Phi^{t}(\rho) \text { is uniformly bounded for } t \in \mathbb{R}\right\},
$$

is a hyperbolic repeller. Following Sjöstrand [74, Appendix c], one can for instance "smoothen" the hard body potential $V_{\Omega}$ associated with the above obstacle problem, and obtain a potential with $J$ "steep bumps" (see Fig. 4), such that the flow is chaotic in some intermediate energy range.

The Schrödinger operator (21) admits a continuous spectrum on $\mathbb{R}_{+}$, but like in the obstacle problem, its truncated resolvent $\chi(P(\hbar)-z)^{-1} \chi$ can be meromorphically continued from the upper to the lower half-plane. The poles $\left\{z_{j}(\hbar)\right\}$ of this continued resolvent form 
a discrete set, which are the resonances of $P(\hbar)$. In general these resonances depend on $\hbar$ in a nontrivial way.

We specifically consider the vicinity of a positive energy $E$ for which the trapped set $K_{E}$ is a hyperbolic set, and ask the same questions as in $\$ 1.2$. The following results are direct analogues of Thm 1 and Thm 2 in this semiclassical setting.

Theorem 3. 57] Consider the semiclassical Hamiltonian $P(\hbar)$ of (21), such that for some energy $E>0$ the flow generated by the Hamiltonian (20) has a hyperbolic trapped set $K_{E}$.

If the topological pressure

$$
\mathcal{P}=\mathcal{P}\left(-\frac{1}{2} \varphi^{+}, \Phi^{t} \uparrow_{K_{E}}\right) \quad \text { is negative, }
$$

then for any $\delta, \epsilon>0$ small enough, there exists $\hbar_{\delta, \epsilon}>0$ such that, for any $\hbar \in\left(0, \hbar_{\delta, \epsilon}\right]$ the operator $P(\hbar)$ does not have resonances in the strip $[E-\delta, E+\delta]-i[0,|\mathcal{P}|-\epsilon]$.

Theorem 4. [74, 78] Let $P(\hbar)$ be a semiclassical Hamilton operator as in Thm 3, and let $2 \nu_{E}+1$ be the upper box dimension of $K_{E}$.

Then, for any $C>0$, the number of resonances of $P(\hbar)$ in the disk $D(E, C \hbar)$, counted with multiplicities, is bounded as follows. For any $\epsilon>0$, there exists $C_{C, \epsilon}, \hbar_{C, \epsilon}>0$ such that

$$
\forall \hbar<\hbar_{C, \epsilon}, \quad \sharp\{\operatorname{Res} P(\hbar) \cap D(E, C \hbar)\} \leq C_{C, \epsilon} \hbar^{-\nu_{E}-\epsilon} .
$$

If $K_{E}$ is of pure dimension, one can take $\epsilon=0$ in the above estimate.

One can generalize the above scattering problems on $\mathbb{R}^{d}$ by considering a Schrödinger operator $P(\hbar)$ of the form (21) on an unbounded Riemannian manifold $(X, g)$ with a "nice enough" geometry near infinity. This geometry should allow to meromorphically extend the truncated resolvent in some strip. For instance, Thm. 3 applies if $X$ is a union of Euclidean infinities outside a compact part [57]. It has been extended by Datchev [20] and Datchev-Vasy [21] to more complicated geometries near infinity, in particular asymptotically Euclidean or asymptotically hyperbolic manifolds. Their strategy is to "glue together" the resolvent estimates of two model manifolds, one with the true, trapping structure in the interaction region but a simple (say, Euclidean) structure near infinity, and the other one with the true infinity but a simple (nontrapping) interaction region. In parallel, Vasy [85] recently developed a new method to analyze the resolvent at high frequency, in a variety of asymptotically hyperbolic geometries.

3.2. Geometric scattering. On a Riemannian manifold $(X, g)$, the classical scattering in absence of potential (one then speaks of geometric scattering) can already be complicated, e.g. chaotic. This is the case, for instance, when the trapped set $K$ lies in a region of negative sectional curvature (see Fig. 4, left). The operator quantizing the geodesic flow is the (semiclassical) Laplace-Beltrami operator on $X, P(\hbar)=-\hbar^{2} \Delta_{X} / 2$. 
One appealing class of examples consists in manifolds $(X, g)$ obtained by quotienting the Poincaré half-space $\mathbb{H}^{n+1}$ (which has uniform curvature -1 ) by certain discrete subgroups $\Gamma$ of the group of isometries on $\mathbb{H}^{n+1}$. Such a manifold $X=\Gamma \backslash \mathbb{H}^{n+1}$ inherits the uniform hyperbolic geometry of $\mathbb{H}^{n+1}$, so that all trajectories are hyperbolic. For a certain type of subgroups $\Gamma$ (called convex co-compact), the manifold $X$ has infinite volume and the trapped set is compact; this trapped set is then automatically a hyperbolic set. This definition of $X$ through group theory leads to remarkable properties of the spectrum of $\Delta_{X}$, which we now summarize (a recent review of the theory in dimension 2 can be found in Borthwick's book [9]).

The absolutely continuous spectrum of $-\Delta_{X}$ consists in the half-line $\left[n^{2} / 4, \infty\right)$, leaving the possibility of finitely many eigenvalues in the interval $(0,1 / 4)$. It is common to write the energy variable as

$$
k^{2}=s(n-s), \quad \text { so that the a.c. spectrum corresponds to } s \in n / 2+i \mathbb{R} .
$$

This parametrization has the following advantage. The resonances of $\Delta_{X}$, parametrized by a discrete set $\left\{s_{j}\right\} \subset\{\operatorname{Re} s<n / 2\}$, exactly correspond to the nontrival zeros of the Selberg zeta function 61]

$$
Z_{X}(s)=\exp \left(-\sum_{p} \sum_{m \geq 1} \frac{1}{m} \operatorname{det}\left(1-P_{p}\right)^{-1 / 2} e^{-s m \ell(p)}\right) .
$$

Here $p$ are the primitive closed geodesics of the flow, and $P_{p}$ is the linearized Poincaré return map around $p$. This shows that the (quantum) resonances are solely determined by the classical dynamics on $K$.

One can show that the rightmost zero of $Z_{X}(s)$ is located at $s_{0}=\delta$, where $\delta>0$ is the Hausdorff dimension of the limit set $\Lambda(\Gamma)$ [60, 82], while all other zeros satisfy

$$
\forall j \neq 0, \quad \operatorname{Re} s_{j}<\delta .
$$

If $\delta \in[n / 2, n]$ ("thick" trapped set), $\delta$ corresponds to the eigenvalue of the ground state of the Laplacian. There may be finitely many other eigenvalues $\left(s_{j} \in[n / 2, \delta)\right)$, while the resonances will be located in the half-space $\{\operatorname{Re} s<n / 2\}$.

If $\delta \in(0, n / 2)$ ("thin" trapped set), all the zeros of $Z_{X}(s)$ correspond to resonances of $\Delta_{X}$, and since the a.c. spectrum corresponds to $\operatorname{Re} s=1 / 2$, the bound (23) shows the presence of a resonance gap. The topological pressure of $\Phi^{t} \uparrow_{K}$ is given by

$$
\mathcal{P}\left(-1 / 2 \varphi^{+}, \Phi^{t} \uparrow_{K}\right)=\delta-n / 2,
$$

so the bound (23) is a (more precise) analogue of the semiclassical gaps in Thms 1 and 3 .

In this geometric setting one can also obtain fractal upper bounds for the number of long-living resonances:

\footnotetext{
${ }^{4}$ Fix one point $x_{0} \in \mathbb{H}^{n+1}$. Then the limit set $\Lambda(\Gamma) \stackrel{\text { def }}{=} \overline{\left\{\gamma \cdot x_{0}, \gamma \in \Gamma\right\}} \cap \partial \mathbb{H}^{n+1}$ actually only depends on the subgroup $\Gamma$.
} 
Theorem 5. 92, 35] Let $X=\Gamma \backslash \mathbb{H}^{n+1}$ be a hyperbolic manifold of infinite volume, with $\Gamma$ a Schottky group. Then the resonances of $\Delta_{X}$ (counted with multiplicities) satisfy

$$
\forall \gamma>0, \exists C_{\gamma}>0, \forall r>1, \quad \sharp\left\{s_{j} \in i[r, r+1]+[n / 2-\gamma, n / 2]\right\} \leq C_{\gamma} r^{\delta} .
$$

Notice that $2 \delta+1$ is the (Hausdorff or Minkowski) dimension of the trapped set $K \subset$ $S^{*} X$. A slightly weaker result was first obtained in 92 in dimension 2 , using microlocal methods similar with those of [74] (see \$4.2). The above result was obtained in [35] by analyzing the Selberg zeta function in terms of a classical expanding map on $\partial \mathbb{H}^{n+1}$, and the corresponding transfer operator. This possibility to rely on purely classical dynamics is specific to the locally homogeneous spaces $\Gamma \backslash \mathbb{H}^{n+1}$.

\section{4. "Massaging" $P(\hbar)$ Into a Proof of the Fractal Weyl upper Bound}

This section presents two consecutive methods, which were used in [78] to prove the fractal Weyl upper bound of Thm 4, that is in the case of a semiclassical Schrödinger operator (21). Both methods consist in "deformations" of the original operator $P(\hbar)$, which can be easily analyzed at the level of the symbols of the operators, so as to draw consequences on the spectra of the deformed operators.

The first method, called "complex scaling", or rather "complex deformation", provides an alternative definition for the resonances of $P(\hbar)$, as the eigenvalues of a nonselfadjoint operator. We remind that resonances were originally obtained as poles of the meromorphic continuation of the truncated resolvent $\chi(P(\hbar)-z)^{-1} \chi$. Each resonance $z_{j}(\hbar)$ is associated with a metastable state $u_{j}(\hbar)$, which is not in $L^{2}$ but satisfies the differential equation $P(\hbar) u_{j}(\hbar)=z_{j}(\hbar) u_{j}(\hbar)$.

4.1. Complex scaling: resonances as spectrum of a nonselfadjoint operator. The "complex scaling" strategy [1, 36] (below we follow the presentation of [76]) consists in deforming the configuration space $\mathbb{R}^{d}$ into a complex contour $\Gamma_{\theta} \subset \mathbb{C}^{d}, \theta \in\left[0, \theta_{0}\right]$, of the form

$$
\Gamma_{\theta} \cap\left\{|x| \leq R_{0}\right\}=\mathbb{R}^{d} \cap\left\{|x| \leq R_{0}\right\}, \quad \Gamma_{\theta} \cap\left\{|x| \geq 2 R_{0}\right\}=\left\{e^{i \theta} x, x \in \mathbb{R}^{d},|x| \geq 2 R_{0}\right\} .
$$

We recall that $B\left(0, R_{0}\right)$ is the interaction region, which contains the support of the potential. The differential operator $P(\hbar)$, when analytically extended on $\Gamma_{\theta}$, is then equivalent with an operator $P_{\theta}(\hbar)$ acting on $\mathbb{R}^{d}$. Outside $B\left(0,2 R_{0}\right)$ this operator is simply given by $-e^{-2 i \theta} \frac{\hbar^{2} \Delta}{2}$ : this shows that $P_{\theta}(\hbar)$ is not selfadjoint on $L^{2}\left(\mathbb{R}^{d}\right)$, and has essential spectrum on the halfline $e^{-2 i \theta} \mathbb{R}_{+}$. More importantly, its spectrum in the cone $\{-2 \theta<\arg (z)<0\}$ is discrete, with eigenvalues $z_{j}(\hbar)$ equal to the resonances of the original operator $P(\hbar)$ (see Fig. 5 ). The associated eigenstates $u_{j, \theta}(\hbar)$ are equal to the metastable states $u_{j}(\hbar)$ inside $B\left(0, R_{0}\right)$, but they are now globally square-integrable.

\footnotetext{
${ }^{5}$ Schottky groups form a certain subclass of convex co-compact groups of isometries.
} 

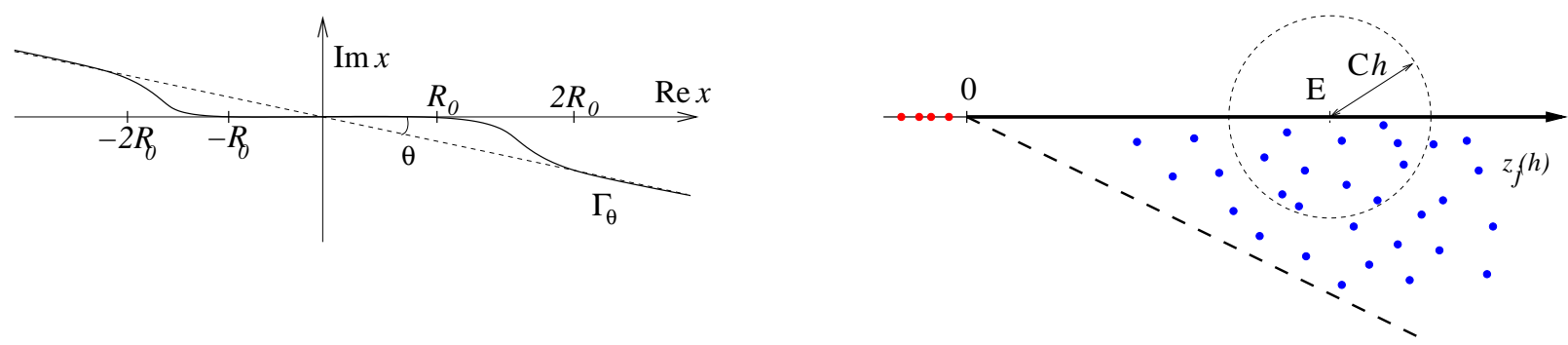

FiguRE 5. Left: deformation of the configuration space $\mathbb{R}^{d}$ into a contour in $\Gamma_{\theta} \subset \mathbb{C}^{d}$. Right: the spectrum of the deformed operator $P_{\theta}(\hbar)$.

Through this deformation, the study of resonances has become a spectral problem for the nonselfadjoint differential operator $P_{\theta}(\hbar)$. We can make advantage of pseudodifferential calculus, that is study the spectrum of $P_{\theta}(\hbar)$ by analyzing its semiclassical symbol $p_{\theta}(x, \xi)$. For $\theta \ll 1$, this symbol takes the form

$$
\begin{aligned}
& p_{\theta}(x, \xi)=p(x, \xi), \quad|x| \leq R_{0} \\
& p_{\theta}(x, \xi)=e^{-2 i \theta} \frac{|\xi|^{2}}{2}, \quad|x| \geq 2 R_{0} .
\end{aligned}
$$

In particular, for any positive energy $E>0$ and $\delta>0$ small, the phase space region

$$
V_{\theta}(\delta) \stackrel{\text { def }}{=}\left\{(x, \xi) \in T^{*} \mathbb{R}^{d},\left|p_{\theta}(x, \xi)-E\right| \leq \delta\right\}
$$

is compact (it is contained inside $T^{*} B\left(0,2 R_{0}\right)$ ). This has for consequence that, for $\hbar$ small enough, $P_{\theta}(\hbar)$ has discrete spectrum in $D(E, C \hbar)$. The compactness of $V_{\theta}(\delta)$ also provides a rough upper bound on the number of eigenvalues near $E$. Heuristically, the number of eigenvalues of $P_{\theta}(\hbar)$ in $D(E, C \hbar)$ is bounded from above by the number of quantum states which can be squeezed in the region $V_{\theta}(C \hbar)$, each state occupying a volume $\sim \hbar^{d}$. This argument can be made rigorous [78, Thm 2], and produces the bound

$$
\text { \# } \operatorname{Spec} P_{\theta}(\hbar) \cap D(E, C \hbar)=\mathcal{O}\left(\hbar^{-d} \operatorname{Vol} V_{\theta}(C \hbar)\right)=\mathcal{O}\left(\hbar^{-d+1}\right) \text {. }
$$

This estimate does not depend on the nature of the dynamics in the interaction region. In case the flow on $K_{E}$ contains stable orbits surrounded by elliptic islands, one can show that this estimate is optimal, by explicitly constructing sufficiently many quasimodes with quasi-energies in $D(E, C \hbar)$ very close to the real axis, and showing that actual eigenvalues must lie nearby (see [83] and references therein).

Remark 4.1. When solving the Schrödinger equation $i \hbar \partial_{t} u=P_{\theta}(\hbar) u$, the negative imaginary part of $P_{\theta}$ acts as an "absorbing" term. Indeed, a wavepacket $u_{0}$ microlocalized near a point $(x, \xi) \in p^{-1}(E),|x| \geq 2 R_{0}$, will be absorbed fast, in the sense that its norm will be reduced by a factor $\sim e^{t \operatorname{Im} p_{\theta}(x, \xi) / \hbar}=e^{-t \sin (2 \theta) E / \hbar}$. Hence, the complex deformation has the effect to absorb the waves propagating outside the interaction region. 
The above complex deformation can be used for any type of potential $V(x)$. In order to refine the counting estimate (26), one strategy [74, 78] consists in a second deformation of the operator $P_{\theta}(\hbar)$, obtained by conjugating it with an appropriate microlocal weight, such as to shrink the region $V_{\theta}(C \hbar)$ to the close vicinity of the trapped set. Eventhough we will present an alternative (yet related) proof of Thm 4 in $\$ 7.2$, we chose to sketch this strategy below, which features the power of pseudodifferential calculus (see the Appendix for a brief introduction).

4.2. Conjugation by an escape function. One constructs by hand an escape function $G \in C_{c}^{\infty}\left(T^{*} X\right)$, which is adapted to the flow $\Phi^{t}$ in some energy layer $p^{-1}([E-\delta, E+\delta])$ in the following way. The function $G$ is required to strictly grow along the flow outside an $\varepsilon$-neighbourhood $\tilde{K}_{E}^{\varepsilon}$ of $\tilde{K}_{E} \stackrel{\text { def }}{=} \cup_{\left|E^{\prime}-E\right| \leq \delta} K_{E^{\prime}}$ (and for $|x| \leq 2 R_{0}$ ). The microlocal weight is then obtained by quantizing this escape function into the operator $G^{w}=\mathrm{Op}_{\hbar}(G)$, and exponentiating: for some factor $t \gg 1$ one defines the deformed operator

$$
P_{\theta, t G}(\hbar) \stackrel{\text { def }}{=} e^{-t G^{w}} P_{\theta}(\hbar) e^{t G^{w}} .
$$

$P_{\theta, t G}$ and $P_{\theta}$ obviously have the same spectrum, but the pseudodifferential calculus (see the Appendix) shows that the former has a symbol of the form

$$
p_{\theta, t G}=p_{\theta}-i \hbar t\{p, G\}+\mathcal{O}\left((\hbar t)^{2}\right),
$$

where the Poisson bracket $\{p, G\}=H_{p} G$ is the derivative of $G$ along the flow generated by $p$. From the construction of $G$, this symbol has a negative imaginary part outside $\tilde{K}_{E}^{\varepsilon}$, showing that $P_{\theta, G}(\hbar)$ is absorbing there. The same volume argument as above then shows that

$$
\text { \# Spec } P_{\theta, t G}(\hbar) \cap D(E, C \hbar)=\mathcal{O}\left(\hbar^{-d} \operatorname{Vol} V_{\theta, t G}(C \hbar)\right) \text {. }
$$

If $\varepsilon>0$ is very small, the above bound is sharper than (26) , because the set $V_{\theta, t G}(C \hbar)$ has a much smaller volume than $V_{\theta}(C \hbar)$. Indeed, the former set is contained inside $\tilde{K}_{E}^{\varepsilon} \cap V_{\theta}(C \hbar)$, the volume of which scales as 6

$$
\operatorname{Vol}\left(\tilde{K}_{E}^{\varepsilon} \cap\{|p(x, \xi)-E| \leq C \hbar\}\right) \asymp \hbar \varepsilon^{2(d-\nu)-2}, \quad \varepsilon, \hbar \ll 1,
$$

where $2 \nu+1$ is the box dimension of $K_{E}$ inside $p^{-1}(E)$. In order to gain a power of $\hbar$ in the right hand side of (28), we need to take $\varepsilon \sim \hbar^{\alpha}$ for some $\alpha>0$, which implies that the escape function $G(x, \xi)$ has to be $\hbar$-dependent. On the other hand, the pseudodifferential calculus leading to (27) is valid only if $G$ belongs to a "good" symbol class, implying that it cannot fluctuate too strongly. As explained in Appendix A.2, the limiting class corresponds to functions fluctuating on distances of order

$$
\varepsilon=\varepsilon(\hbar) \sim \hbar^{1 / 2} .
$$

Injecting (30) into (29) leads to the fractal Weyl upper bound of Thm 4 ,

\footnotetext{
${ }^{6}$ here we assume $\tilde{K}_{E}$ is of "pure" Minkowski dimension. In the general case one needs to replace dim by $\operatorname{dim}+\epsilon$ for any arbitrary $\epsilon>0$.
} 
The construction of an optimal escape function $G$ is a bit tricky, it uses the hyperbolicity of the flow on $\tilde{K}_{E}$. To give a schematic idea, let us consider the simple example of the model Hamiltonian [16]

$$
p=\xi_{1}+x_{2} \xi_{2} \quad \text { on } T^{*}\left(S^{1} \times \mathbb{R}\right)
$$

for which $K_{E}$ consists in a single hyperbolic periodic orbit $\left\{\xi_{1}=E, x_{2}=\xi_{2}=0\right\}$. An "optimal" escape function is then

$$
G_{1}(x, \xi)=\log \left(\varepsilon^{2}+x_{2}^{2}\right)-\log \left(\varepsilon^{2}+\xi_{2}^{2}\right) \Longrightarrow H_{p} G_{1}=\frac{x_{2}^{2}}{\varepsilon^{2}+x_{2}^{2}}+\frac{\xi_{2}^{2}}{\varepsilon^{2}+\xi_{2}^{2}},
$$

with the scaling (30). Indeed, the gradient $H_{p} G_{1} \geq 1$ for $|(x, \xi)| \geq C \varepsilon$, while the function remains in a reasonable symbol class.

In the case of a fractal trapped set $K_{E}$, the escape function $G$ will locally have a structure similar with $G_{1}$ near $K_{E}$, except that the coordinates $x_{2}, \xi_{2}$ are replaced by functions more or less measuring the distance from the incoming/outgoing tails $K_{E}^{\mp}$.

\section{OPEN QUANTUM MAPS AND QUANTUM MONODROMY OPERATORS}

In this section we introduce open quantum maps, which are toy models used to study the distribution of quantum resonances, especially in chaotic situations. These toy models present the advantage to be easy to implement numerically. Besides, the recent introduction of quantum monodromy operators in the context of chaotic scattering establishes a direct link between the resonances of a Schrödinger operator, and this particular family of open quantum maps (quantizing a Poincaré return map of the classical flow).

5.1. Open quantum maps. In this section we introduce the open maps, and their quantizations, the open quantum maps.

An open map is a symplectic diffeomorphism $\kappa: V \mapsto \kappa(V)$, where $V$ and $\kappa(V)$ are bounded open subsets of a symplectic manifold $\Sigma$, which locally looks like $T^{*} \mathbb{R}^{d}$. The map $\kappa$ is "open" because we assume that $\kappa(V) \neq V$, so there exist points $\rho \in V$ such that $\kappa(\rho)$ has no further image; we interpret it by saying that $\kappa(\rho)$ has "fallen in the hole", or has "escaped to infinity" (this interpretation will become clearer when we specifically treat Poincaré maps). By time inversion, the map $\kappa^{-1}: \kappa(V) \rightarrow V$ is also an open map, and points in $V \backslash \kappa(V)$ have escaped to infinity "in the past".

This escape phenomenon naturally leads to the notions of incoming/outgoing tails and trapped set: similarly as in Eqs. (9), we define

$$
\mathcal{K}^{\mp} \stackrel{\text { def }}{=}\left\{\rho \in V \cup \kappa(V), \kappa^{ \pm n}(\rho) \in V, \forall n>0\right\}, \quad \mathcal{K}=\mathcal{K}^{-} \cap \mathcal{K}^{+} .
$$

We will assume that $\mathcal{K}$ is compact and at finite distance from the boundary $\partial V$. As in the case of flows, we will say that the open map $\kappa$ is chaotic iff the dynamics generated by $\kappa$ on $\mathcal{K}$ is hyperbolic and complex, in the sense of $₫ 2$. We will see in $\$ 5.3$ that the Poincaré 
return map of a chaotic scattering flow is a chaotic open map. Still, it is not difficult to directly construct chaotic open maps, e.g. by starting from a chaotic "closed" map $\tilde{\kappa}$ (diffeomorphism) on $\Sigma$, and restricting it on a subset $V$.

What do we call a "quantization" of the map $\kappa$ ? In the case $\Sigma=T^{*} \mathbb{R}^{d}$, it is a family of operators $(\mathcal{M}(\hbar))_{\hbar \rightarrow 0}$ on $L^{2}\left(\mathbb{R}^{d}\right)$, with the following asymptotic properties when $\hbar \rightarrow 0$.

First, $\mathcal{M}(\hbar)=\mathcal{M}(\alpha, \hbar)$ should be an $\hbar$-Fourier Integral Operator (FIO) associated with $\kappa$, with symbol $\alpha \in C_{c}^{\infty}(V)$ (see Appendix $\$$ A.3 for more details). For any smooth observable $a \in C_{c}^{\infty}\left(T^{*} \mathbb{R}^{d}\right)$, the quantized observable $\mathrm{Op}_{\hbar}(a)$ is transformed as follows when conjugated by $\mathcal{M}(\hbar)$ :

$$
\mathcal{M}(\hbar)^{*} \mathrm{Op}_{\hbar}(a) \mathcal{M}(\hbar)=\mathrm{Op}_{\hbar}(b)+\mathcal{O}_{L^{2} \rightarrow L^{2}}\left(\hbar^{\infty}\right) .
$$

The function $b(x, \xi ; \hbar)$ is a semiclassical symbol in $S^{0}(V)$ supported on supp $\alpha$, and admitting the expansion

$$
b=|\alpha|^{2} \times a \circ \kappa+\mathcal{O}(\hbar) .
$$

For $\hbar$ small enough, $\mathcal{M}(\alpha, \hbar)$ is uniformly bounded, with

$$
\|\mathcal{M}(\alpha, \hbar)\|=\|\alpha\|_{\infty}+\mathcal{O}(\hbar) .
$$

Equation (32) is a form of Egorov theorem (see (75)). It implies that $\mathcal{M}(\alpha, \hbar)$ transforms a wavepacket $u_{0}$ microlocalized at a point $\left(\rho_{0}\right) \in V$ into a wavepacket $\mathcal{M}(\alpha, \hbar) u_{0}$ microlocalized at $\kappa\left(\rho_{0}\right)$, with a norm modified by

$$
\frac{\left\|\mathcal{M}(\alpha, \hbar) u_{0}\right\|}{\left\|u_{0}\right\|}=\left|\alpha\left(\rho_{0}\right)\right|+\mathcal{O}(\hbar)
$$

The coefficient $\left|\alpha\left(\rho_{0}\right)\right|$ can be interpreted as an absorption (resp. gain) factor if $\left|\alpha\left(\rho_{0}\right)\right|<1$ (resp. $\left|\alpha\left(\rho_{0}\right)\right|>1$ ). The compact support of $\alpha$ shows that the particles outside supp $\alpha$ are fully absorbed.

To call $\mathcal{M}(\alpha, \hbar)$ an open quantum map, one furthermore requires that it is microlocally unitary inside $V$. This means that the above ratio of norms should be $1+\mathcal{O}\left(\hbar^{\infty}\right)$, for any state $u_{0}$ microlocalized inside $V$. For this to happen, the symbol $|\alpha(\rho)|$ needs to be a smoothed version of the characteristic function $\mathbb{1}_{V}$ on $V$. Typically, one can consider a family of neighbourhoods $\mathcal{K} \Subset W_{\hbar} \subset V$ converging to $V$, say, $W_{\hbar}=\{\rho \in V$, $\operatorname{dist}(\rho, \complement V) \leq$ $r(\hbar)\}$, e.g. with $r(\hbar) \sim|\log \hbar|^{-1}$, and require that the symbol $\alpha$ satisfies

$$
\alpha(\rho)=0 \text { outside } V, \quad|\alpha(\rho)|=1 \text { inside } W_{\hbar} \text {, and }|\alpha(\rho)| \in[0,1] \text { inbetween. }
$$

Such $\alpha$ depends on $\hbar$, but in a mild enough way (see the Appendix $\S$ A.2). To insist on the regularity of $\alpha$, we will call such an operator a smooth open quantum map.

\footnotetext{
7 The notation $A(\hbar)=\mathcal{O}_{L^{2} \rightarrow L^{2}}\left(\hbar^{\infty}\right)$ is a shorthand for the fact that for any $N \geq 0,\|A(\hbar)\|_{L^{2} \rightarrow L^{2}}=$ $\mathcal{O}\left(\hbar^{N}\right)$ when $\hbar \rightarrow 0$.
} 
5.2. Open quantum maps of finite rank. A priori, the operators $\mathcal{M}(\alpha, \hbar)$ have infinite rank, even though they have a finite essential rank, of the order of $\hbar^{-d} \operatorname{Vol}(\operatorname{supp} \alpha)$, when $\hbar \rightarrow 0$. This corresponds to the dimension of a subspace of states which are not fully absorbed. For practical reasons it can be convenient to replace $\mathcal{M}(\hbar)$ by a finite rank operator $M(\hbar)$, by composing $\mathcal{M}(\hbar)$ with a projector $\Pi(\hbar)$ microlocally equal to the identity in some neighbourhood of $\operatorname{supp} \alpha$, and of rank $\sim C \hbar^{-d}$. One then obtains a family of operators

$$
M(\hbar) \stackrel{\text { def }}{=} \mathcal{M}(\hbar) \Pi(\hbar)=\mathcal{M}(\hbar)+\mathcal{O}_{L^{2} \rightarrow L^{2}}\left(\hbar^{\infty}\right) .
$$

Such a projection onto a subspace of finite dimension will be used in the construction of quantum monodromy operators in $\$ 5.4$.

A practical way to construct an open map is to start from a symplectomorphism $\tilde{\kappa}$ defined on a compact phase space $\Sigma$, say the torus $\mathbb{T}^{2 d}=\mathbb{R}^{2 d} / \mathbb{Z}^{2 d}$, and then restrict it to a proper open subset $V \Subset \mathbb{T}^{2 d}$, that is take $\kappa=\tilde{\kappa} \uparrow_{V}$. There exist recipes to quantize the "closed" map $\tilde{\kappa}$ into a quantum map [22], that is a family $(U(\hbar))_{\hbar \rightarrow 0}$ of unitary operators acting on the family of quantum spaces $\left(\mathcal{H}_{\hbar}\right)_{\hbar \rightarrow 0}$ associated with $\mathbb{T}^{2 d}$, and enjoying a Egorov property similar with (32), with $\alpha \equiv 1$. The spaces $\mathcal{H}_{\hbar}$ have dimensions $\sim(2 \pi \hbar)^{-d}$ due to the compactness of $\mathbb{T}^{2 d}$ (with the constraint $(2 \pi \hbar)^{-1} \in \mathbb{N}$ ).

To "open" this quantum map, one can truncate $U(\hbar)$ by a projector quantizing $\mathbb{1}_{V}$, and get the operator

$$
M(\hbar)=U(\hbar) \Pi(\hbar) .
$$

The rank of $\Pi(\hbar)$ then scales as $\hbar^{-d} \operatorname{Vol}(V)$. Once again, we speak of a smooth open quantum map if $\Pi(\hbar)$ is not a strict projector, but rather a "quasiprojector" which is also the quantization of a "good" symbol $\alpha$, like in (35). This choice allows to avoid diffraction problems near the boundary of $V$.

The above construction was implemented for various chaotic maps on the 2-dimensional torus, chosen such that the open map $\kappa$ admits a hyperbolic trapped set. In all cases the cutoff $\Pi(\hbar)$ was a sharp projector (in position or momentum). The first such map was, to my knowledge, the "kicked rotor" with absorption [8]; it was already aimed at studying the statistics of quantum lifetimes, defined in terms of the spectrum $\left\{\lambda_{j}(\hbar)\right\}$ of $M(\hbar)$ :

$$
e^{-\tau_{j}(\hbar) / 2}=\left|\lambda_{j}(\hbar)\right|, \quad j=1, \ldots, \operatorname{rank}(M(\hbar)) .
$$

This formula shows that the "long living" spectrum of $M(\hbar)$, say $\left\{\lambda_{j}(\hbar)\right\} \cap\{|\lambda| \geq r\}$ for some fixed $r>0$, is seen as a model for the resonances of $P(\hbar)$ in some box $[E-C \hbar, E+$ $C \hbar]-i[0, \gamma \hbar]$, with the connection $r \equiv e^{-\gamma}$. Further studies lead to the verification of the fractal Weyl law [71, 55, 56] (see \$6.1).

Such discrete time models have several advantages. Firstly, the long time dynamics of the classical map $\kappa$ is sometimes easy to analyze; this is the case for instance for the open baker's map studied in [55, 56, which we will explicitly describe in \$6.1.1. Secondly, 
the corresponding open quantum maps are often very explicit matrices, which can be numerically diagonalized, much more easily so than Schrödinger operators like $P_{\theta}(\hbar)$. A variant of the quantum baker's map even lends itself to an analytical treatment (see $\$ 6.1 .2)$. Third, the quantum monodromy operators establish a connection between a family of open quantum maps and a "physical" scattering flow. (see \$5.4). To explain the construction of the monodromy operators we need to recall the definition of Poincaré sections associated with a Hamiltonian flow.
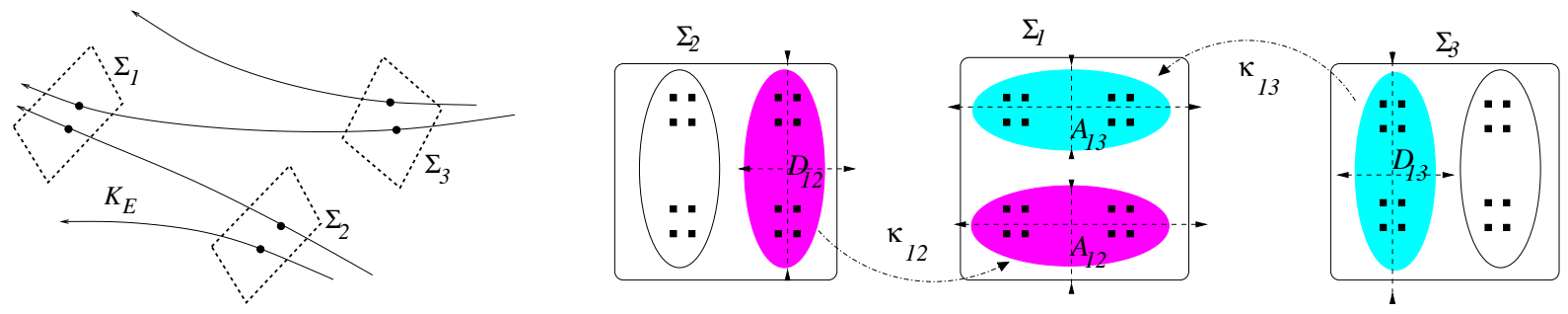

FIgURE 6. Left: schematic representation of a Poincaré section $\boldsymbol{\Sigma}$ near the trapped set $K_{E}$. Right: the induced return map $\kappa=\left(\kappa_{i j}\right)$ on $\boldsymbol{\Sigma}$. Vertical/horizontal axes indicate the stable/unstable directions, and the trapped set $\mathcal{K}$ is sketched by the black squares.

5.3. Poincaré sections. We are back to the setting of $\oint_{2}$, with a Hamiltonian flow $\Phi^{t}$ on $T^{*} \mathbb{R}^{d}$ (more generally $T^{*} X$ for some manifold $X$ ). Given $E>0$ a noncritical energy, a Poincaré section near the trapped set $K_{E}$ is a finite union of hypersurfaces $\boldsymbol{\Sigma}=\sqcup_{i=1}^{I} \Sigma_{i}$ in $p^{-1}(E)$, uniformly transverse to the flow, such that for each point $\rho$ sufficiently close to $K_{E}$ the trajectory $\Phi^{t}(\rho)$ intersects $\Sigma$ (in the future and the past) after a uniformly bounded time (Fig. 6). This property allows to define

a return map $\kappa: V \subset \Sigma \rightarrow \kappa(V) \subset \Sigma, \quad$ and a return time $\tau: V \subset \Sigma \rightarrow \mathbb{R}_{+}$,

where $V$ is a neighbourhood of the reduced trapped set

$$
\mathcal{K} \stackrel{\text { def }}{=} K_{E} \cap \Sigma=\sqcup_{i=1}^{I} \mathcal{K}_{i}
$$

Since the flow $\Phi^{t}$ is symplectic on $T^{*} X$, the Poincaré section $\Sigma$ can be given a natural symplectic structure, which is preserved by $\kappa$. Notice the dimensional reduction: $\Sigma$ has dimension $2 d-2$. The flow $\Phi^{t}$ in the neighbourhood of $K_{E}$ is fully described by the pair $(\kappa, \tau)$. In particular, $\kappa \uparrow_{\mathcal{K}}$ is hyperbolic if $\Phi^{t} \uparrow_{K_{E}}$ is so. Analyzing the ergodic properties of such a hyperbolic map has proved easier than directly analyzing the flow. Indeed, the thermodynamic formalism, which allows to construct nontrivial invariant measures, and analyze their ergodic properties, is based on such a Poincaré reduction [10].

Since $\boldsymbol{\Sigma}$ is a union of disjoint hypersurfaces $\Sigma_{i}$ locally equivalent with $T^{*} \mathbb{R}^{2 d-2}$, the map $\kappa$ can be seen as a collection of symplectic maps $\kappa_{i j}: D_{i j} \subset \Sigma_{j} \mapsto \Sigma_{i}$, where $D_{i j}$ consists of the points in $\Sigma_{j}$, the trajectories of which next intersect $\Sigma_{i}$. An open quantum map 
associated with $\kappa$ is then an operator valued matrix $\mathcal{M}(\hbar)=\left(\mathcal{M}_{i j}(\hbar)\right)_{i, j=1, \ldots, I}$, such that $\mathcal{M}_{i j}(\hbar)=0$ if $D_{i j}=\emptyset$, otherwise $\mathcal{M}_{i j}(\hbar)$ is an open map quantizing $\kappa_{i j}$. The operator $\mathcal{M}(\hbar)$ acts on $L^{2}\left(\mathbb{R}^{d-1}\right)^{I}$, and satisfies a vector-valued Egorov property similar with (32), where $a=\left(a^{i}\right)_{i=1, \ldots, I}$ and $b=\left(b^{i}\right)_{i=1, \ldots, I}$ are observables on $\boldsymbol{\Sigma}$. One can also consider open quantum maps of finite rank $M(\hbar)$, as in (36).

5.4. Quantum monodromy operators. It turns out that, under a mild condition on the trapped set $K_{E}$, there exists a family of quantum maps (more precisely, of FIOs) associated with the Poincaré map $\kappa$, which allows to directly recover the resonance spectrum of the quantum Hamiltonian $P(\hbar)$.

Theorem 6. 53, 54] Let $P(\hbar)$ be as in Thm. 3, and assume that the trapped set set $K_{E}$ is totally disconnected transversely to the flous.

Alternatively, take $P(\hbar)=-\frac{\hbar^{2} \Delta}{2}$ the Dirichlet Laplacian outside J convex obstacles satisfying the no-eclipse condition, and take $E=1 / 2$.

Consider a Poincaré section $\boldsymbol{\Sigma}=\sqcup_{i=1}^{I} \Sigma_{i}$ transverse to $\Phi^{t}$ near $K_{E}$. Then, there exists a family of quantum monodromy operators $(M(z, \hbar))_{z \in D(E, C \hbar)}$ on $L^{2}\left(\mathbb{R}^{d-1}\right)^{I}$, with the following properties:

(i) $M(E, \hbar)$ is an open quantum map quantizing $\kappa$, of finite rank $\hbar^{-d+1}$.

(ii) $M(z, \hbar)$ depends holomorphically in $z \in D(E, C \hbar)$, and

$$
M(z, \hbar)=M(E, \hbar) \mathrm{Op}_{\hbar}\left(e^{-i(z-E) \tau / \hbar}\right)+\mathcal{O}\left(\hbar^{1-\epsilon}\right),
$$

where $\tau$ is the return time (smoothly continued outside $V$ ).

(iii) the resonances of $P(\hbar)$ in $D(E, C \hbar)$ are the roots (with multiplicities) of the equation

$$
\operatorname{det}(1-M(z, \hbar))=0 \text {. }
$$

The properties $(i),(i i)$ ensure that for all $z \in D(E, C \hbar), M(z, \hbar)$ remains an FIO associated with $\kappa$, but for $z \notin \mathbb{R}$ it is no more unitary near $\mathcal{K}$.

The crucial property (iii) exhibits the connection between the spectrum of $M(z, \hbar)$ and the resonances of $P(\hbar)$. It has transformed a linear spectral problem $\left(P_{\theta}(\hbar)-z\right) u=0$, into a problem $M(z, \hbar) v=v$ of finite rank, depending nonlinearly in $z$. The construction of the monodromy operators $M(z, \hbar)$ is not unique, and rather implicit. Roughly speaking, each component $M_{i j}(z, \hbar)$ is obtained by expressing the microlocal solutions to the equation $\left(P_{\theta}(\hbar)-z\right) u=0$ near $\mathcal{K}_{j}$ in terms of their "local transverse data" $v_{j} \in L^{2}\left(\mathbb{R}^{d-1}\right)$, using a choice of coordinates near $\Sigma_{i}$. The microlocal solutions $u$ can be continued up to $\Sigma_{i}$, where they are analyzed in terms of local transverse data $v_{i} . M_{i j}(z, \hbar)$ is defined as the operator mapping $v_{j}$ to $v_{i}$ : this explains the denomination of "monodromy operator" (see Fig. (7). The main technical difficulty consists in transforming this microlocal characterization into a globally defined, finite rank operator.

\footnotetext{
${ }^{8}$ This condition is probably generic within the family of chaotic scattering flows we are considering. It can be relaxed a bit, see [53]
} 


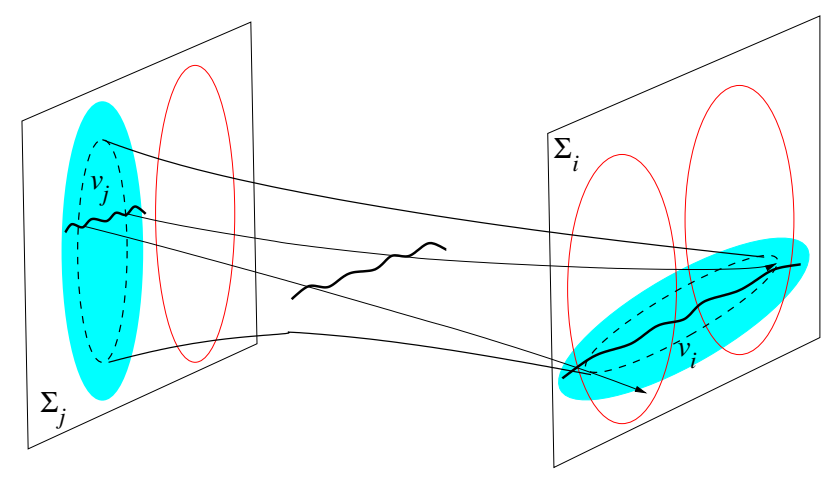

FIGURE 7. Schematic construction of the monodromy operator $M_{i j}(z, \hbar)$, by "following" a microlocal solution of $(P(\hbar)-z) u=0$ from a neighbourhood of $\Sigma_{j}$ to a neighbourhood of $\Sigma_{i}$.

A monodromy operator had been constructed, and used to study the resonance spectrum of a scattering operator $P(\hbar)$, in the case where the trapped set consists in a single hyperbolic orbit [31]. In a different framework, a microlocal form of monodromy operator associated with an isolated periodic orbit was used in [77] to compute the contribution of this orbit to Gutzwiller's semiclassical trace formula.

In the physics literature, Bogomolny [5] formally defined a "quantum transfer operator" $T(E, \hbar)$ associated with the Hamiltonian $P(\hbar)$ of a closed system: this operator quantizes the return map through a certain spatial hypersurface, and in the semiclassical limit the eigenvalues of $P(\hbar)$ are (formally) given by the roots of the equation $\operatorname{det}(1-T(E, \hbar))=0$. This approach was adapted by Doron and Smilansky to study the spectrum of closed Euclidean billiards [25], and was also implemented in a nonsemiclassical setting by Prosen 65 .

In the case of the scattering by $J$ convex obstacles, similar operators were constructed [30, 37]. Here the section $\boldsymbol{\Sigma}$ is "selected" by the setting: it consists in the union of the cotangent bundles of the obstacle boundaries, $\Sigma_{i}=T^{*} \partial \mathcal{O}_{i}$. To each obstacle $\mathcal{O}_{i}$ one associates a Poisson operator $H_{i}(k): C^{\infty}\left(\partial \mathcal{O}_{i}\right) \mapsto C^{\infty}\left(\mathbb{R}^{d} \backslash \mathcal{O}_{i}\right)$, such that

$$
\forall v \in C^{\infty}\left(\partial \mathcal{O}_{i}\right), \quad\left\{\begin{array}{l}
\left(\Delta+k^{2}\right) H_{i}(k) v=0 \quad \text { on } \mathbb{R}^{d} \backslash \mathcal{O}_{i}, \\
H_{i}(k) v \text { is outgoing, } \\
\left(H_{i}(k) v\right)\left\lceil\partial \mathcal{O}_{i}=v .\right.
\end{array}\right.
$$

Then, the scattering problem by the $J$ obstacles can be expressed in terms of the "quantum boundary map" $\mathcal{M}(k)=\left(\mathcal{M}_{i j}(k)\right)_{i, j=1, \ldots, J}$ defined by

$$
\mathcal{M}_{i j}(k): C^{\infty}\left(\partial \mathcal{O}_{j}\right) \mapsto C^{\infty}\left(\partial \mathcal{O}_{i}\right), \quad \begin{cases}\mathcal{M}_{i j}(k)=0, & i=j \\ \mathcal{M}_{i j}(k) v=\left(H_{j}(k) v\right) \Gamma_{\partial \mathcal{O}_{i}} & i \neq j\end{cases}
$$


In the high frequency limit $k \rightarrow \infty$, and away from the "glancing orbits", the operator $\mathcal{M}_{i j}(k)$ has the structure of an open quantum map associated with the boundary map of the billiard flow, . In [54] we show how to reduce these boundary operators $\mathcal{M}(k)$ to finite rank monodromy operators $M(k)$, which have the properties expressed in the above theorem (as explained in §3, the correspondence with the semiclassical formalism reads $\left.\hbar \sim|k|^{-1}, z=\hbar^{2} k^{2} / 2=1 / 2+\mathcal{O}(\hbar)\right)$.

\section{From fractal Weyl upper bound to fractal Weyl LaW ?}

In our attempts to address the question (1) in $\$ 1.2$, we have so far only obtained upper bounds for the number of resonances. Lower bounds are more difficult to derive, due to the fact that the spectral problem we are dealing with is effectively nonselfadjoint. Upper bounds are generally obtained by first counting the singular values of some operator related with $P_{\theta}(\hbar)$, which is a selfadjoint spectral problem; after controlling the distribution of singular values one can then apply Weyl's inequalities 9 to bound (from above) the number of eigenvalues of $P_{\theta}(\hbar)$.

The difficulty to obtain a lower bound (that is, ensure that there are indeed about as many eigenvalues as what is permitted by the upper bound) may be traced to the possible high sensitivity of the spectrum w.r.t. perturbations. So far, the only access to lower bound is provided by some form of Gutzwiller's (or Selberg's) trace formula. Using this strategy, lower bounds on the number of resonances have been obtained in the case of convex co-compact manifolds $X=\Gamma \backslash \mathbb{H}^{n+1}$ (we use the notations of $\S 3.2$ ).

Theorem 7. [34, 62] Let $X=\Gamma \backslash \mathbb{H}^{n+1}$, with $\Gamma$ a convex co-compact and torsion-free subgroup. Then, for any small $\epsilon>0$, there exists $\gamma_{\epsilon}>0$ such that

$$
\sharp\left\{s_{j} \in i[0, r]+\left[-\gamma_{\epsilon}, n / 2\right]\right\}=\Omega\left(r^{1-\epsilon}\right) \quad \text { when } r \rightarrow \infty .
$$

The proof of this lower bound uses an exact, Selberg-like trace formula, which connects the resonance spectrum on one side, with a sum over the closed geodesics on the other side. Applying a well-chosen test function on this trace formula, one exhibits a singularity on the "geodesics side", which implies (on the "spectral side") the presence of many resonances.

We notice a gap between this lower bound and the upper bound of Thm 5, which implies that the left hand side is bounded above by $C_{\gamma} r^{1+\delta}$, with $\delta>0$ the dimension of the limit set. In [34] the authors conjecture that the actual number of resonances in the strip is of the order of the fractal upper bound. Similar conjectures for various other systems can be split into two forms.

\footnotetext{
${ }^{9}$ Let $\left(\lambda_{i}\right)$ (resp. $\left(s_{i}\right)$ ) be the eigenvalues (resp. singular values) of a compact operator, ordered by decreasing moduli. Then, for any $j \geq 1, \sum_{i=1}^{j}\left|\lambda_{i}\right| \leq \sum_{i=1}^{j} s_{i}$.

$10_{\text {the notation }} f(r)=\Omega(g(r))$ means that $\frac{f(r)}{g(r)}$ takes arbitrarily large values when $r \rightarrow \infty$.
} 
Definition 6.1. Let $P(\hbar)$ be a Schrödinger operator as in (21), and assume that for some $E>0$ the trapped set $K_{E}$ is a hyperbolic repeller of pure Minkowski dimension $1+2 \nu$. We define the weak, resp. strong form of fractal Weyl law conjecture as follows.

(i) Weak form. For $C, \gamma>0$ large enough (for a very weak form, take $C \sim \delta \hbar^{-1}$ ), there exists $C_{\gamma}>0, \hbar_{C, \gamma}>0$ such that

$$
\sharp\{\operatorname{Res} P(\hbar) \cap[E-C \hbar, E+C \hbar]-i[0, \gamma \hbar]\} \geq C C_{\gamma} \hbar^{-\nu}, \quad \forall \hbar<\hbar_{C, \gamma} .
$$

(ii) Strong form. There exists an increasing function $F: \mathbb{R}_{+} \mapsto \mathbb{R}_{+}$, nonidentically vanishing, such that, for any $C, \gamma>0$,

$$
\sharp\{\operatorname{Res} P(\hbar) \cap[E-C \hbar, E+C \hbar]-i[0, \gamma \hbar]\}=C F(\gamma) \hbar^{-\nu}+o\left(\hbar^{-\nu}\right), \quad \text { when } \hbar \rightarrow 0 \text {. }
$$

In order to test either form of the conjecture, resonance spectra have been numerically computed for the three types of systems: the 3-bump potential [44, 45] or a modified Hénon-Heiles Hamiltonian [66], the 3-disk scattering on the plane[47] (see Fig. 8), or a scattering by 4 spheres on the 3 -dimensional space [26], and several convex co-compact surfaces 35$]_{11}^{11}$. In all cases, the counting was compatible with the fractal Weyl law, although the convergence to the asymptotic behaviour was difficult to ascertain. More recently, attemps have been made to extract the long living resonances of the 3-disk scattering system from an experimental signal on a microwave (quasi)-2d billiard by the Marburg group [43]. Yet, computing high frequency resonances in such an experiment presents many difficulties: a noisy and discrete signal, the presence of antennas perturbing the ideal system, the difficulties to reach sufficiently high frequencies, and the delicate implementation of the harmonic inversion method used to extract the "true" resonances.

The fractal Weyl conjecture was actually much easier to test numerically for the toy model of open quantum maps.

6.1. Fractal Weyl law for open quantum maps. As explained above, in the quantum map framework the distribution of long-living states is studied by fixing some radius $r>0$, and counting the number of eigenvalues $\lambda_{j}(\hbar)$ of $M(\hbar)$ in the annulus $\{r \leq|\lambda| \leq 1\}$. This task is easy to implement numerically for operators (matrices) $M(\hbar)$ of reasonable dimensions. Schomerus and Tworzydło implemented it on the kicked rotor [71] in a strongly chaotic régime12, with a sharp opening along a vertical strip. A very good agreement with the strong fractal Weyl law was observed: most eigenvalues accumulate near $\lambda=0$, while a small fraction of them have moduli $\geq r$. Their numerics hint at the existence of a nontrivial profile function $r \in(0,1] \mapsto F(r) \geq 0$, such that

$$
\forall r>0, \quad \sharp\{\operatorname{Spec} M(\hbar) \cap\{|\lambda| \geq r\}\}=F(r) \hbar^{-\nu}+o\left(\hbar^{-\nu}\right),
$$

\footnotetext{
${ }^{11}$ In the last two cases, resonances were obtained by computing the zeros of the Selberg/Gutzwiller zeta functions: this procedure exactly provides the resonances in the convex co-compact case, while in the obstacle case the zeros are believed to be good approximations of the actual resonances.

${ }^{12}$ As far as I know, the chaoticity of the kicked rotor has not been proved rigorously, but seems plausible in view of numerics.
} 


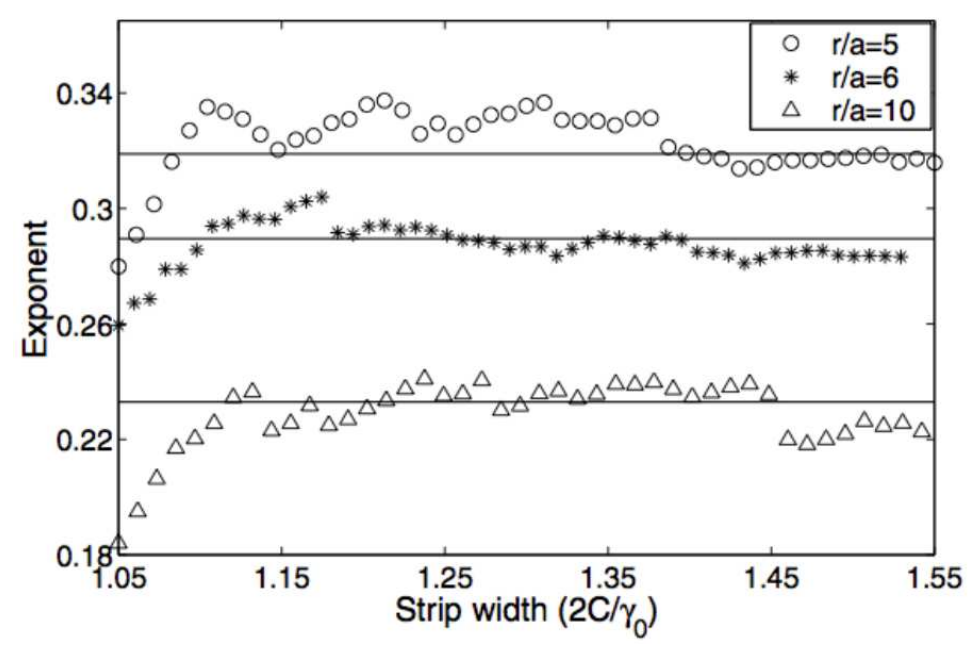

Figure 8. Check of the fractal Weyl law for the the scattering by 3 disks of radii a located in an equilateral triangle of sidelength $r$, for 3 values of the ratio $r / a$. In each case a fractal exponent $\nu(C)$ was extracted from counting the resonances in a long strip of depth $C$ for various values of $C$ larger than $2 \gamma_{c l}$, and compared with the geometrical exponent $\nu$ (horizontal lines). Reprinted figure with permission from W. Lu, S. Sridhar, M. Zworski, Phys. Rev. Lett. 91, 154101 (2003). Copyright 2003 by the American Physical Society.

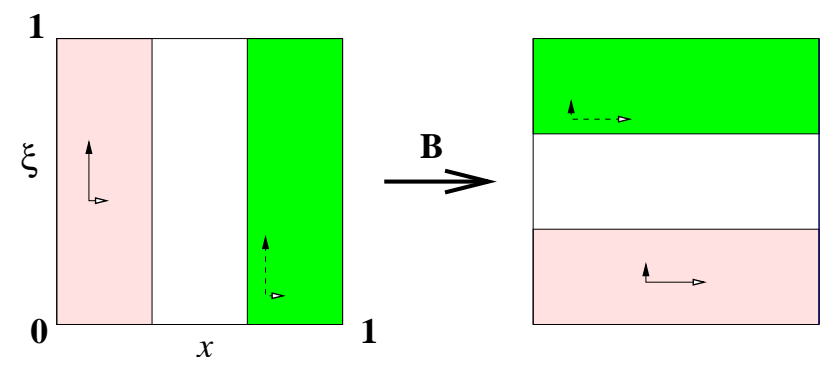

FiguRE 9. Sketch of the symmetric 3-baker's map, with a hole in the central rectangle.

with $\nu=\operatorname{dim} \mathcal{K} / 2$.

6.1.1. The open baker's map. The spectra of several types of quantum open baker's maps were analyzed in [55, 56, 52]. Let us recall the definition and basic properties of this family of chaotic maps on $\mathbb{T}^{2}$.

A baker's map $\tilde{\kappa}$ is defined by splitting $\mathbb{T}^{2}$ into $D \geq 2$ "Markov rectangles" $R_{i}=\left\{x_{i} \leq\right.$ $\left.x<x_{i+1}, 0 \leq \xi<1\right\}, i=0, \ldots, D-1, x_{0}=0, x_{D}=1$, and mapping the points in $R_{i}$ as 
follows:

$$
(x, \xi) \in R_{i} \mapsto \tilde{\kappa}(x, \xi)=\left(\frac{x-x_{i}}{\ell_{i}}, \ell_{i} \xi+x_{i}\right), \quad \text { with } \ell_{i} \stackrel{\text { def }}{=} x_{i+1}-x_{i}
$$

Since $\ell_{i}<1$, the stable/unstable directions are the vertical/horizontal axes. This map is invertible, but discontinuous along the boundaries $\partial R_{i}$. There is an obvious symbolic dynamics: to a point $\rho=(x, \xi)$ one associates the sequence $\cdots \epsilon_{-1} \cdot \epsilon_{0} \epsilon_{1} \cdots, \epsilon_{j} \in\{0, \ldots, D-$ $1\}$, such that $\tilde{\kappa}^{j}(\rho) \in R_{\epsilon_{j}}$ for each time $j \in \mathbb{Z}$. Conversely, to any bi-infinite sequence corresponds a point $\rho$, and this map is "almost" one-to-one 13 .

In order to take advantage of this symbolic dynamics, the opening $\mathbb{T}^{2} \backslash V$ was chosen to consist in the union of $D-n$ of the Markov rectangles, $0<n<D$. The trapped set $\mathcal{K}$ is then easy to describe (see Fig. 11): it consists in the sequences $\boldsymbol{\epsilon}$ with all $\epsilon_{j} \in I=\left\{i_{1}, \ldots, i_{n}\right\}$ the set of "kept rectangles". This set is the cartesian product Can $\times$ Can, where Can is a Cantor set on the unit interval; the Hausdorff or Minkowski dimension $\nu$ of Can is explicitly given by the only real root of the equation

$$
\ell_{i_{1}}^{s}+\ell_{i_{2}}^{s}+\cdots+\ell_{i_{n}}^{s}=1 .
$$

For instance, if we keep $n$ rectangles in the symmetric $D$-baker's map, we get $\nu=\frac{\log n}{\log D}$.

Assume the $\ell_{i}$ are rational. For quantum dimensions $N=(2 \pi \hbar)^{-1}$ such that $N \ell_{i}$ are all integer, the "closed map" $\tilde{\kappa}$ is quantized according to the recipes of Balasz-Voros or Saraceno [4, 67], namely by the unitary matrix

$$
U(\hbar)=U_{N}=F_{N}^{-1}\left(\begin{array}{lll}
F_{N \ell_{0}} & & \\
& \ddots & \\
& & F_{N \ell_{D-1}}
\end{array}\right),
$$

where $F_{*}$ is the $*$-dimensional discrete Fourier transform. The quantization of the open map $\kappa$ is simply obtained by projecting out the $D-n$ blocks $F_{N \ell_{j}}$ corresponding to the opening.

A strong form of fractal Weyl law was observed for an asymmetric baker's map (see Fig. (10). For symmetric baker's maps (that is, taking $x_{i}=i / D$, see Fig 91), the fractal scaling seems satisfied, but we observed that different profile functions occurred along different geometric sequences $\left(N=N_{o} D^{k}\right)_{k \geq 0}$, a manifestation of the number theoretic properties of such symmetric maps. Apart from these specific number theoretic issues, the form of the profile function for both the kicked rotor and the baker maps looks similar: $F(r)$ decays regularly from $r \approx 0$ and approximately vanishes around some value $r_{\max }<1$, with a "dip" before $r_{\max }$, showing a (mild) peak of the density around some value $r_{\text {peak }} \leq r_{\max }$. The position of this peak seems close to the classical decay rate, $r_{\text {peak }} \approx e^{-\gamma_{c l} / 2}[72]$.

\footnotetext{
${ }^{13}$ The defect of injectivity comes from points with sequences ending by infinite strings of 0 , on either end. For instance, the point $(0,0)$ can be represented by the constant sequences $\overline{0}$ or $\overline{D-1}$.
} 

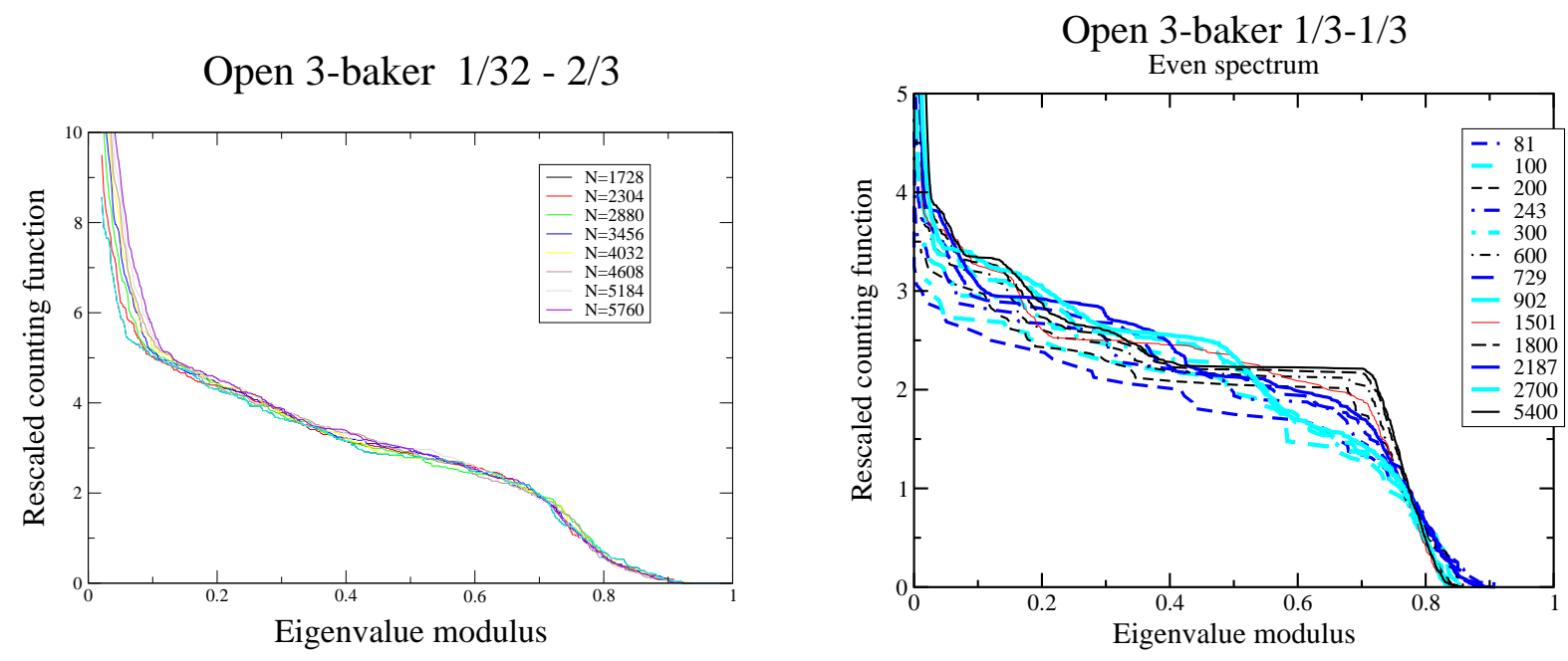

FIGURE 10. Rescaled counting function for an asymmetric (left) and a symmetric (right) open 3-baker opened by removing the central rectangle (the fractions in the title denote the contraction factors $\ell_{0}, \ell_{2}$ ). In both cases the rescaling consists in dividing the counting function by the factor $N^{\nu}$, where $\nu=\operatorname{dim}(\mathcal{K}) / 2$. Reprinted from $[52]$.

A random matrix model Ansatz was proposed in [71] to account for the profile function $F(r)$, hinting at a certain "universality" of this profile, but the validity of this Ansatz remains unclear.

6.1.2. A solvable model satisfying the fractal Weyl law. A "toy-of-the-toy" model was studied in [55, 56], in the form of a nonstandard quantization of the symmetric $D$-baker's map. In the case of quantum dimension $N=D^{k}$, this quantization amounts to replacing the discrete Fourier transform $F_{N}$ by the Walsh-Fourier transform, that is the Fourier transform on $\left(\mathbb{Z}_{D}\right)^{k}$. This quantization $M_{N}=M_{D^{k}}$ of the open baker's map then admits a very simple tensor product representation on the Hilbert space $\mathcal{H}_{N} \equiv\left(\mathbb{C}^{D}\right)^{\otimes k}$.

This property allows to explicitly compute the spectrum of $M_{N}$ : the latter is given in terms of the $D \times D$ matrix $\Omega_{D}$, obtained by removing from the inverse Fourier transform $F_{D}^{*}$ the $(D-n)$ columns corresponding to the opening. Generally, this matrix has a $(D-n)-$ dimensional kernel and $n$ nontrivial eigenvalues, which are the eigenvalues of the $n \times n$ square matrix $\widetilde{\Omega}_{D}$ extracted from $\Omega_{D}$. This results in $n^{k}=N^{\nu}$ nontrivial eigenvalues (counted with multiplicities) for $M_{N}$, and proves the strong form of fractal Weyl law (42). The profile function $F(r)$ has the form of a step function at some value $r_{c}=\left|\operatorname{det} \widetilde{\Omega}_{D}\right|^{1 / n}$. 
Yet, we noticed in [55, Remark 5.2] that for some choices of parameter:14, the spectrum of $\widetilde{\Omega}_{D}$ may present an "accidental" extra kernel. In that case, the counting function is $\mathcal{O}\left(\hbar^{-\nu^{\prime}}\right)$ with $\nu^{\prime}<\operatorname{dim}(\mathcal{K}) / 2$, so even the weak fractal Weyl law fails. This accidental degeneracy seems due to the very special tensor product structure of the Walsh quantization, and should be nongeneric. It was checked [29] that this accidental degeneracy disappears if we modify the matrix $M_{N}$ by multiplying it by a diagonal matrix of random, or even deterministic phases. Nevertheless, this problem may indicate that any attempt to prove the fractal Weyl law in any setting might require some genericity assumption, or the introduction of some random parameters in the system.

\section{INTERPRETATION OF THE FRACTAL WEYL UPPER BOUND FOR OPEN QUANTUM MAPS}

After reviewing the numerical (and some analytical) results regarding the optimality of the fractal Weyl upper bound, let us present some heuristics for this upper bound in the case of open quantum maps, as well as a rigorous proof for smooth open quantum maps. Both use a reduction of the dynamics to an effective propagator of "minimal rank", which accounts for the quantum dynamics near the trapped set.

7.1. Heuristic explanations. A semiclassical mechanism explaining the fractal Weyl upper bound for an open chaotic map $\kappa$ has been put forward in [71. The idea is that the (essential) generalized kernel of $M(\hbar)$ is larger than its kernel (associated with the opening), due to the presence of (approximate) Jordan blocks reflecting the transient classical dynamics of the points which wander through $V$ before escaping.

For any $n \geq 1$, consider the sets $D_{n} \in \mathbb{T}^{2}$ of points escaping before the time $n$. This set consists in a union of finitely many connected components $D_{n, j}$, most of which look like "thin tubes" aligned along the stable manifolds when $n \gg 1$ (see Fig. 11, left). The widths of the thin tubes decay like $e^{-n \lambda}$, where $\lambda$ is the Lyapunov exponent. For fixed $n$, one can associate to each component $D_{n, j}$ a quantum subspace $\mathcal{H}_{\hbar, n, j}$ of dimension $(2 \pi \hbar)^{-1} \operatorname{Vol}\left(D_{n, j}\right)$. The subspaces $\mathcal{H}_{\hbar, n, j}$ are semiclassically almost orthogonal to each other, so that $\mathcal{H}_{\hbar, n}=\bigoplus_{j} \mathcal{H}_{\hbar, n, j}$ has dimension $(2 \pi \hbar)^{-1} \operatorname{Vol}\left(D_{n}\right)$.

The semiclassical evolution implies that any state $u \in \mathcal{H}_{\hbar, n}$ will be absorbed when iterated up to time $n$ :

$$
\left\|M(\hbar)^{n} u\right\|=\mathcal{O}\left(\hbar^{\infty}\right)\|u\|, \quad \forall u \in \mathcal{H}_{\hbar, n} .
$$

This property implies that the long-living eigenvalues of $M(\hbar)$ are essentially the same as those of $M(\hbar) \Gamma_{\mathcal{H}_{\hbar, n}^{\perp}}$, so their number is at most $(2 \pi \hbar)^{-1}\left(1-\operatorname{Vol}\left(D_{n}\right)\right)$.

\footnotetext{
${ }^{14}$ It is the case, for instance, if we kill the second and fourth rectangles from the symmetric 4-baker: in that case, $M_{N}$ has a single, simple nontrivial eigenvalue. One can cook up an even more dramatic example (with $D=16, n=2$ ), for which $\operatorname{Spec}\left(M_{N}\right)=\operatorname{Spec}\left(\widetilde{\Omega}_{D}\right)=\{0\}$.
} 

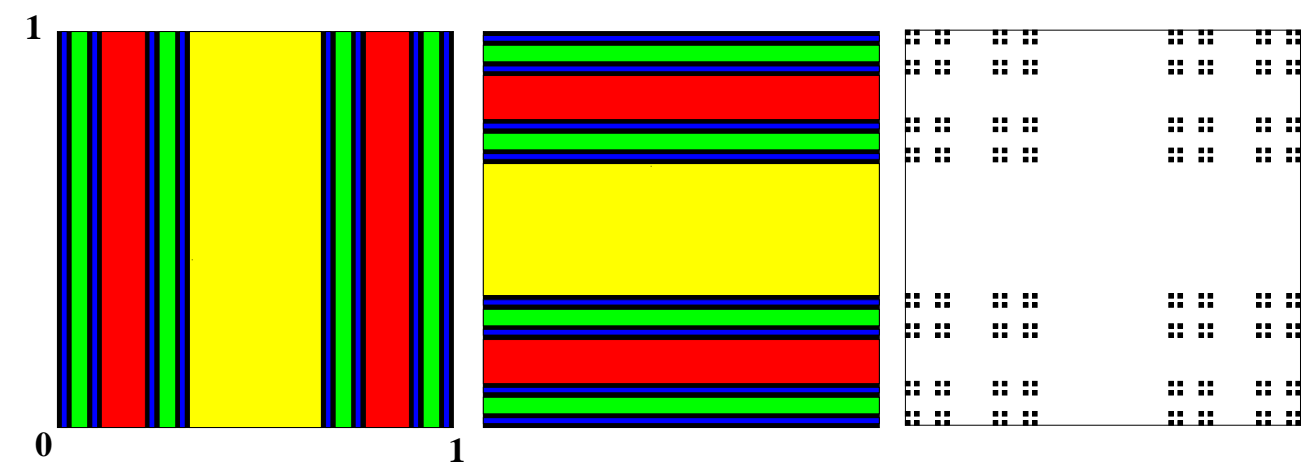

Figure 11. Construction of the trapped set (right) and its incoming (left) and outgoing (center) tails for the symmetric 3-baker. Each color corresponds to a specific escape time (in the futur or past) $n=1, \ldots, 4$, the trapped set (and its tails) being approximated by union of black intervals/squares.

When $n \gg 1$ the set $\complement D_{n}$ of the points with escape times $>n$ is a small neighbourhood of the incoming tail $\mathcal{K}^{-}$(see Fig. 11), and

$$
\operatorname{Vol}\left(\complement D_{n}\right) \sim e^{-n \gamma_{c l}}
$$

where $\gamma_{c l}>0$ is the classical decay rate. To get a fractal upper bound, one needs to push the time $n$ to infinity in a $\hbar$-dependent way. As long as $n$ is smaller than the Ehrenfest time

$$
T_{E h r} \sim \frac{\log 1 / \hbar}{\lambda_{\max }}, \quad \text { where } \lambda_{\max } \text { is the largest expansion rate, }
$$

the tubes $D_{n, j}$ have volumes $\gg \hbar$, which means that one can associate nontrivial quantum subspaces $\mathcal{H}_{\hbar, n, j}$ to $D_{n, j}$. Ignoring problems due to the boundaries of $D_{n, j}$, let us push the above argument up to $n=T_{E h r}$ : the bound on the number of long-living eigenvalues then reads

$$
(2 \pi \hbar)^{-1} \operatorname{Vol}\left(\complement D_{T_{E h r}}\right) \sim \hbar^{-1+\frac{\gamma_{c l}}{\lambda_{\max }}} .
$$

This argument is not optimal if the hyperbolicity is not homogeneous, the exponent $1-\frac{\gamma_{c l}}{\lambda_{\max }}$ being larger than $\nu=\operatorname{dim}(\mathcal{K}) / 2$. Still, the above reasoning clearly exhibits the connection between resonance counting and small ( $\hbar$-dependent) neighbourhoods of the trapped set $\mathcal{K}$ (or its tail $\mathcal{K}^{-}$). It also shows the (approximate) Jordan structure of $M(\hbar)$, quantum analogue of the transient dynamics before $T_{E h r}$. An alternative approach to the problem was adopted by Novaes et al. in [59]. There the quantum dynamics was projected by hand on a "minimal" quantum subspace microlocalized near the trapped set, resulting in an effective spectral problem of smaller dimension. The minimal subspace was generated by a certain number $\left(\sim C \hbar^{-\nu}\right)$ of (Left and Right) scar functions $u_{n}^{L}, u_{n}^{R}$, that are quasimodes of $M(\hbar)$, resp. $M(\hbar)^{*}$, microlocalized along periodic orbits of periods $T \leq T_{E h r}$. The authors 

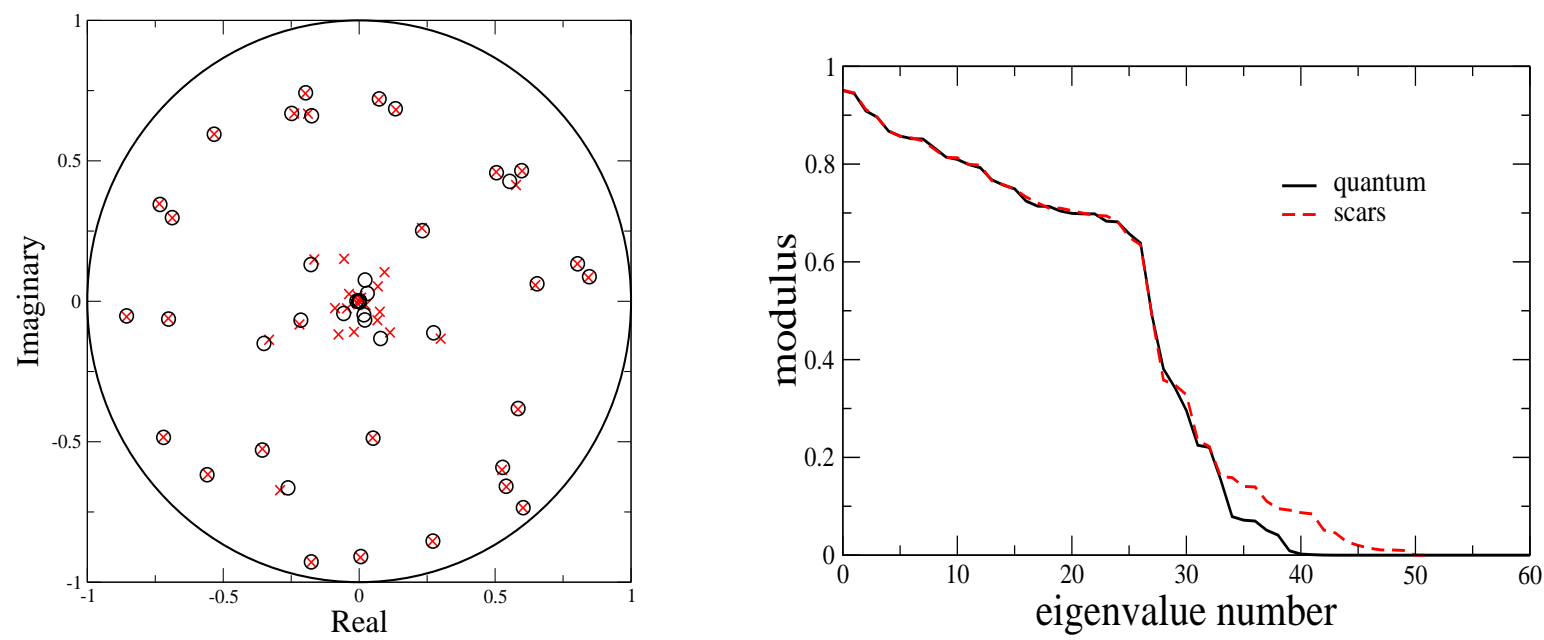

Figure 12. Left: spectrum of the quantum open baker's map ( $D=3$ symmetric, $N=81$ ), by diagonalizing the matrix $M_{N}$ (red crosses) or by using the "scar matrix" $E(z)$ from periodic orbits of length $\leq 5$, and solving (45) (black circles). Right: the corresponding radial countings (compared with Fig. 10, the two axes should be exchanged). Reprinted figure with permission from M. Novaes et al., Phys. Rev. E 80 035202(R) (2009). Copyright 2009 by the American Physical Society.

used these scar functions to construct the generalized eigenvalue problem

$$
\operatorname{det}(E(\lambda))=0, \quad E_{m n}(\lambda) \stackrel{\text { def }}{=}\left\langle u_{n}^{L},\left(I-\lambda^{-1} M(\hbar)\right) u_{m}^{R}\right\rangle,
$$

and checked that the solutions of this problem accurately approximated the long living spectrum of $M(\hbar)$ (see Fig. 12).

The operator $E(\lambda)$, representing the quantum dynamics on $\mathcal{K}$, is an (approximate) effective propagator for the quantum map $M(\hbar)$, and can be considered to be of minimal rank, meaning that no further reduction seems possible. Another advantage of this matrix $E_{m n}(\lambda)$ is its sparsity: since quasimodes are localized near periodic orbits, each quasimode $u_{m}^{R}$ (resp. iterated quasimode $M(\hbar) u_{m}^{R}$ ) interferes only with a few quasimodes $u_{n}^{L}$.

7.2. A proof of the fractal Weyl upper bound. A rigorous proof of the fractal upper bound for smooth open quantum map 15 can be obtained using similar ideas. Notice that this smoothness condition excludes the open quantum maps used in most numerical studies [71, 57, 72].

\footnotetext{
${ }^{15}$ see $₫\left[5.2\right.$ the map $\kappa$ is smooth, and the quasiprojector $\Pi(\hbar)=\mathrm{Op}_{\hbar}(\alpha)$ is a "nice" pseudodifferential operator
} 
Theorem 8. [54 Consider $\kappa: V \mapsto \kappa(V)$ a (smooth) open map such that its trapped set $\mathcal{K} \Subset V$ is a hyperbolic repeller of upper Minkowski dimension $2 \nu$, and a corresponding smooth quantum map $\mathcal{M}(\hbar)$, more generally a FIO $\mathcal{M}(\alpha, \hbar)$ with symbol $\alpha \in C_{c}^{\infty}(V)$.

Then, for any $r>0$ and any small $\epsilon>0$, there exists $C_{r, \epsilon}, \hbar_{r, \epsilon}>0$ s.t.

$$
\forall \hbar<\hbar_{r, \epsilon}, \quad \sharp\{\operatorname{Spec}(\mathcal{M}(\hbar)) \cap\{|\lambda| \geq r\}\} \leq C_{r, \epsilon} \hbar^{-\nu-\epsilon} .
$$

If $\mathcal{K}$ is of pure dimension, one can take $\epsilon=0$.

The same estimate holds for $M(\hbar)$ an open quantum map of finite rank.

The proof is an "exponentiation" of the case of Schrödinger operators presented in $\$ 4.2$. One constructs an escape function $G(x, \xi)$ on $V \cup \kappa(V)$, such that

$$
G \circ \kappa-G \geq 1 \text { outside an } \varepsilon \text {-neighbourhood } \mathcal{K}^{\varepsilon} \text { of the trapped set } \mathcal{K} \text {. }
$$

To ensure that $G$ is a "nice" symbol, this neighbourhood must have a width $\varepsilon \gtrsim \hbar^{1 / 2}$. One then quantizes this escape function into an operator $G^{w}$, and uses the latter to conjugate the quantum map $\mathcal{M}(\hbar)$ into

$$
\mathcal{M}_{t G}(\hbar) \stackrel{\text { def }}{=} e^{-t G^{w}} \mathcal{M}(\hbar) e^{t G^{w}}, \quad t \gg 1 .
$$

The Egorov theorem and the pseudodifferential calculus show that $\mathcal{M}_{t G}(\hbar)$ is still a FIO, but with a modified symbol $\alpha_{t G} \approx \alpha e^{-t(G \circ \kappa-G)}$. For $t \gg 1$ the escape property (46) ensures that $\mathcal{M}_{t G}(\hbar)$ strongly suppresses the states microlocalized outside $\mathcal{K}^{\varepsilon}$. One can thus construct a quantum subspace $\mathcal{H}_{\varepsilon}$ of dimension $\sim C \hbar^{-\nu}$ microlocalized on $\mathcal{K}^{\varepsilon}$, such that

$$
\left\|\left(I-\Pi_{\varepsilon}\right) \mathcal{M}_{t G}\right\| \ll 1
$$

where $\Pi_{\varepsilon}$ is the orthogonal projector on $\mathcal{H}_{\varepsilon}$.

From this remark, one can easily show that the number of long living singular values of $\mathcal{M}_{t G}$ is $\mathcal{O}\left(\hbar^{-\nu}\right)$, and get a similar bound for its eigenvalues using Weyl's inequalities.

We present an alternative argument, which has the advantage to apply as well to the case of monodromy operators. The property (47) shows that, for any $\lambda \in \mathbb{C}$ with $|\lambda|>r$, the operator

$$
E(\lambda) \stackrel{\text { def }}{=}\left(I-\lambda^{-1} \Pi_{\varepsilon} \mathcal{M}_{t G}\right)-\lambda^{-2} \Pi_{\varepsilon} \mathcal{M}_{t G}\left(I-\Pi_{\varepsilon}\right) \mathcal{M}_{t G}\left[I-\lambda^{-1}\left(I-\Pi_{\varepsilon}\right) \mathcal{M}_{t G}\right]^{-1}
$$

is well-defined, and the second term on the RHS is a small correction compared with the first one. Notice the similarity of the first term with the operator in (45). A little algebra shows that the long living eigenvalues of $\mathcal{M}_{t G}(\hbar)$ can be exactly obtained by solving

$$
\operatorname{det}(E(\lambda))=0, \quad|\lambda| \geq r .
$$

This confers to $E(\lambda)$ the role of an effective Hamiltonian for the quantum map $\mathcal{M}(\hbar)$. This operator "minimally" captures the long time quantum evolution, which is "supported" on $\mathcal{K}$. Applying Jensen's formula, one then shows that the number of roots of (49) is bounded from above by $\operatorname{dim} \mathcal{H}_{\varepsilon} \sim C \hbar^{-\nu}$. 
The same argument can be used to count the roots of $\operatorname{det}(I-M(z, \hbar))$, with $M(z, \hbar)$ the quantum monodromy operators of Thm 6. One defines an effective Hamiltonian $E(z)$ as in (48), replacing everywhere $\lambda^{-1} M(\hbar)$ by $M(z, \hbar)$ [54]. This leads to a proof of Thms 2 , and an alternative proof for Thms 4 and 5 (under the assumption that the trapped set $K_{E}$ is totally disconnected transversely to the flow).

\section{Resonance GaP FOR OPEn QUANTUm MAPS AND MONODROMY OPERATORS}

Let us now turn to Question (2), namely the criterion for a resonance gap expressed in Theorems 1 and 3. Below we will state the corresponding result for open quantum maps and quantum monodromy operators, which can also be used to prove these theorems.

Theorem 9 (Spectral gap for open quantum maps). Let $\kappa: V \Subset \Sigma \mapsto \kappa(V)$ be a smooth open map with hyperbolic trapped set $\mathcal{K}$, and $\mathcal{M}(\alpha, \hbar)$ an FIO associated with $\kappa$ with symbol $\alpha \in C_{c}^{\infty}(V)$ nonzero near $\mathcal{K}$.

Then, for any small enough $\epsilon>0$, there exists $\hbar_{\epsilon}>0$ such that the spectral radii of the FIOs $\mathcal{M}(\alpha, \hbar)$ satisfy

$$
\forall \hbar \leq \hbar_{\epsilon}, \quad r_{s p}(\mathcal{M}(\alpha, \hbar)) \leq \exp \left\{\mathcal{P}\left(-\varphi^{+} / 2+\log |\alpha|, \kappa\lceil\mathcal{K})+\epsilon\right\} .\right.
$$

Here $\varphi^{+}(\rho) \in C(\mathcal{K})$ is the logarithm of the unstable Jacobian of $\kappa$, and $\mathcal{P}(\bullet)$ is the topological pressure. The case of open quantum maps corresponds to taking $\alpha \equiv 1$ on $\mathcal{K}$.

The same bound holds if we replace $\mathcal{M}(\alpha, \hbar)$ by a finite rank truncation $M(\alpha, \hbar)$ as in $\$ 5.2$.

We notice that the norm estimate (34) implies the bound

$$
r_{s p}(\mathcal{M}(\alpha, \hbar)) \leq\|\alpha\|_{\infty}+\mathcal{O}(\hbar) .
$$

This bound may be sharper than (50), depending on both $\kappa$ and $\alpha$. For instance, if $\alpha \equiv 1$ near $\mathcal{K}$, then (50) is sharper than (51) iff the pressure $\mathcal{P}\left(-\varphi^{+} / 2, \kappa \Upsilon_{\mathcal{K}}\right)$ is negative, a condition satisfied only provided $\mathcal{K}$ is "thin enough".

Before sketching the proof of this theorem in the next section, let us explain how it can be used to prove Thms 1 and 3 . Eq. (39) shows that the monodromy operator $M(z, \hbar)$ associated with a scattering operator $P(\hbar)$ (or an obstacle problem) has the form of an FIO associated with a Poincaré return map $\kappa$, with symbol

$$
\alpha_{z}(\rho)=e^{-i \zeta \tau(\rho)}+\mathcal{O}(\hbar) \quad \text { near } \mathcal{K}, \quad \zeta \stackrel{\text { def }}{=} \frac{z-E}{\hbar},
$$

where $\tau(\rho)$ is the return time. So, the relevant pressure is $\mathcal{P}\left(-\varphi^{+} / 2-\operatorname{Im} \zeta \tau, \kappa\lceil\mathcal{K})\right.$.

Let us assume that the root $s_{0}$ of the equation

$$
\mathcal{P}\left(-\varphi^{+} / 2-s \tau, \kappa \Upsilon_{\mathcal{K}}\right)=0 \quad \text { satisfies } s_{0}<0 .
$$

Then, if we take $\operatorname{Im} \zeta \geq s_{0}+\tilde{\epsilon}$ for some $\tilde{\epsilon}>0$, the pressure $\mathcal{P}\left(-\varphi^{+} / 2-\operatorname{Im} \zeta \tau\right)$ will be negative, and the above theorem implies that, for $\hbar$ small enough, $r_{s p}(M(z, \hbar))<1$. 
In turn, this bound implies (through Thm 6) that there are no resonance in the strip $D(E, C \hbar) \cap\left\{\operatorname{Im} \zeta>s_{0}+\tilde{\epsilon}\right\}$. Finally, the theory of Axiom A flows [10] shows that $s_{0}$ is equal to the topological pressure of the flow:

$$
s_{0}=\mathcal{P}\left(-\varphi_{\Phi}^{+} / 2, \Phi^{t} \uparrow K_{E}\right),
$$

where $\varphi_{\Phi}^{+}$is the unstable Jacobian of the flow, Eq. (13). Hence, the condition $s_{0}<0$ is equivalent with the conditions in Thms 1 and 3 .

8.1. Proof of the resonance gap in terms of the topological pressure. The proof of the spectral bound (50) is analogous to the case of the Schrödinger flow treated in [57]. Let us assume that the trapped set $\mathcal{K}$ is totally disconnected, which is the case if we want to apply the result to monodromy operators. This restriction is not necessary, but it simplifies the proof a little.

To obtain an upper bound on the spectral radius of $\mathcal{M}(\alpha, \hbar)$ (which we will denote by $\mathcal{M}(\hbar)$ from now on), the usual strategy is to estimate $\left\|\mathcal{M}(\hbar)^{n}\right\|$, with $n \gg 1$. Inspired by classical dynamical methods, we will proceed by splitting $\mathcal{M}(\hbar)^{n}$ into many components, each one being associated with a "pencil" of classical trajectories. The topological pressure will then naturally arise when summing over all the "pencils".

Let us be more precise. Using the assumption that $\mathcal{K}$ is totally disconnected, for any small $\epsilon>0$ we may consider a Markov cover $\left(V_{a}\right)_{a \in A_{1}}$ of the trapped set, such that the open sets $V_{a}$ have diameters at most $\epsilon$. The Markov property means the following: the sets $V_{a}$ are disjoint, and from them we may construct the transition matrix

$$
T_{a^{\prime} a}= \begin{cases}1 & , \quad V_{a} \cap \kappa^{-1}\left(V_{a^{\prime}}\right) \neq 0, \\ 0, & \text { otherwise }\end{cases}
$$

Then, for any sequence of symbols $\boldsymbol{\alpha}=\alpha_{0} \alpha_{1} \cdots \alpha_{n-1}$, the set

$$
V_{\boldsymbol{\alpha}} \stackrel{\text { def }}{=} V_{\alpha_{0}} \cap \kappa^{-1}\left(V_{\alpha_{0}}\right) \cap \cdots \cap \kappa^{-n+1}\left(V_{\alpha_{n-1}}\right)
$$

is nonempty if and only if, at all steps $j=0, \ldots, n-2$, one has $T_{\alpha_{j+1} \alpha_{j}}=1$. The set $V_{\boldsymbol{\alpha}}$ consists of the initial points $\rho$ which share the same "symbolic history" for times $0 \leq j \leq n-1$; it makes up a "pencil" of trajectories.

To each set $V_{a}$ we associate the weight

$$
w_{a}=\max _{\rho \in \mathcal{K} \cap V_{a}} e^{-\varphi^{+}(\rho) / 2}|\alpha(\rho)|,
$$

and consider the weighted transition matrix $T_{a^{\prime} a}^{w}=T_{a^{\prime} a} w_{a}$. The topological pressure appearing in (50) is then approximated by the largest (Perron-Frobenius) eigenvalue of the matrix $T^{w}$ :

$$
\mathcal{P}\left(-\varphi^{+} / 2+\log |\alpha|, \kappa\left\lceil_{\mathcal{K}}\right)=\lim _{\epsilon \rightarrow 0} \log \lambda_{P F}\left(T^{w}\right)\right.
$$


We may complete this Markov cover into an open cover of $V$,

$$
V \subset \cup_{a \in A} V_{a}, \quad A=A_{1} \cup A_{+} \cup A_{-},
$$

such that, for some time $n_{o}$, all sets $V_{a_{-}}, a_{-} \in A_{-}$(resp. $V_{a_{+}}, a_{+} \in A_{+}$) escape in the hole before the time $n_{o}$ in the backwards (resp. forward) evolution, see Fig. 13, To the

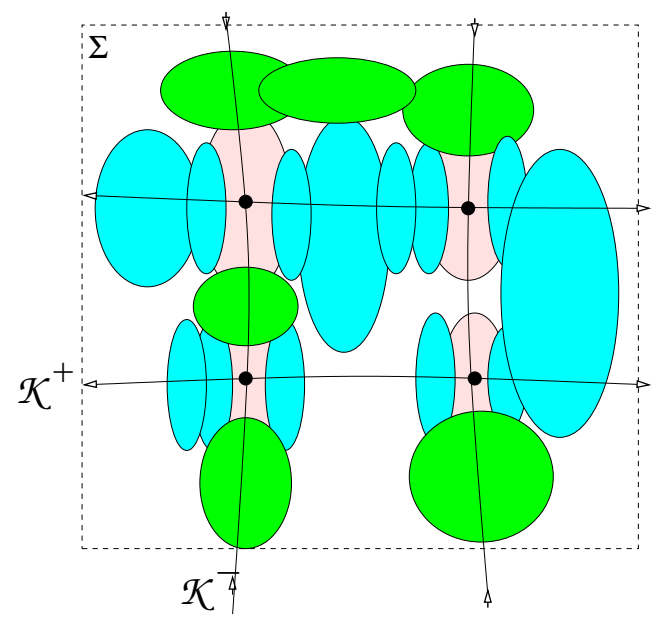

FigurE 13. Sketch of the open cover $\left(V_{a}\right)_{a \in A_{1}}$ of the trapped set (pink), completed by the open sets $\left(V_{a}\right)_{a \in A_{+}}$(cyan) and $\left(V_{a}\right)_{a \in A_{-}}$(green) away from $\mathcal{K}$. Black lines indicate the tails $\mathcal{K}^{ \pm}$and black circles the trapped set.

cover $\left(V_{a}\right)$ we associate a smooth partition of unity of the phase space $\boldsymbol{\Sigma}$, namely a finite collection of cutoffs $\chi_{a} \in C_{c}^{\infty}\left(V_{a},[0,1]\right)$, satisfying

$$
\sum_{a \in A} \chi_{a} \equiv 1 \quad \text { in some neighbourhood of } V,
$$

and add a component in the hole, $\chi_{\infty}=1-\sum_{a \in A} \chi_{a}$ to get a full partition of unity. This smooth partition of unity is then quantized into a quantum partition

$$
I d=\sum_{a \in A \cup \infty} \mathrm{Op}_{\hbar}\left(\chi_{a}\right),
$$

which is used to split the iterated propagator $\mathcal{M}(\hbar)^{n}$ into components:

$$
\mathcal{M}(\hbar)^{n}=\sum_{|\boldsymbol{\alpha}|=n} \mathcal{M}_{\boldsymbol{\alpha}}, \quad \mathcal{M}_{\boldsymbol{\alpha}}=\mathcal{M}_{\alpha_{n-1}} \mathcal{M}_{\alpha_{n-2}} \cdots \mathcal{M}_{\alpha_{0}}, \quad \mathcal{M}_{a} \stackrel{\text { def }}{=} \mathcal{M}(\hbar) \mathrm{Op}_{\hbar}\left(\chi_{a}\right)
$$

8.1.1. Analyzing the components $\mathcal{M}_{\boldsymbol{\alpha}}$. The advantage of this decomposition is to obtain an upper bound for the individual components $\mathcal{M}_{\boldsymbol{\alpha}}$ which is sharper than the obvious bound

$$
\left\|\mathcal{M}_{\boldsymbol{\alpha}}\right\| \leq\left(\|\alpha\|_{\infty}+\mathcal{O}(\hbar)\right)^{n} .
$$


For this, we use our knowledge of the classical dynamics. From Egorov's theorem, we know that for any finite sequence $\boldsymbol{\alpha}$, the element $\mathcal{M}_{\boldsymbol{\alpha}}=\mathcal{O}\left(\hbar^{\infty}\right)$ unless the set $V_{\boldsymbol{\alpha}} \neq \emptyset$; the sequence $\boldsymbol{\alpha}$ is then called admissible. Also, any sequence containing $a=\infty$ leads to a negligible term.

As a result, since the sets $V_{a_{-}}\left(\right.$resp. $V_{a_{+}}$) escape before the time $n_{0}$ in the past (resp. in the future), we deduce that for any $n>2 n_{0}$ the only nonnegligible components $\mathcal{M}_{\boldsymbol{\alpha}}$ must be of the form

$$
\boldsymbol{\alpha}=\boldsymbol{\alpha}_{+} \boldsymbol{\alpha}^{(1)} \boldsymbol{\alpha}_{-}, \quad\left|\boldsymbol{\alpha}_{-}\right|=\left|\boldsymbol{\alpha}_{+}\right|=n_{0}, \quad \boldsymbol{\alpha}^{(1)} \in A_{1}^{n-2 n_{0}} \quad \text { admissible. }
$$

In view of this property, for $n \gg 1$ we may restrict ourselves to the admissible sequences $\boldsymbol{\alpha} \in A_{1}^{n}$, that is replace $\mathcal{M}(\hbar)$ by $\mathcal{M}_{A_{1}} \stackrel{\text { def }}{=} \sum_{a \in A_{1}} \mathcal{M}_{a}$.

8.1.2. Acting on a Lagrangian state: a hyperbolic dispersive estimate. We now want to use the hyperbolicity of $\kappa$ near $\mathcal{K}$. If we apply the FIO $\mathcal{M}_{\alpha_{0}}$ to a Lagrangian 16 state $u_{0}$ supported by a Lagrangian manifold $\Lambda_{\alpha_{0}}$ transverse to the stable direction $E^{-}$, the state will expand along the unstable direction. If the resulting state spreads outside $V_{\alpha_{1}}$, cutting it through $\mathrm{Op}_{\hbar}\left(\chi_{\alpha_{1}}\right)$ will reduce its norm by a finite factor, while the output state will again be a WKB state along a Lagrangian $\Lambda_{\alpha_{1} \alpha_{0}}$ transverse to $E^{-}$. This phenomenon repeats itself, and leads to the following hyperbolic dispersive estimate:

$$
\left\|\mathcal{M}_{\boldsymbol{\alpha}} u_{0}\right\| \leq C w_{\boldsymbol{\alpha}}, \quad w_{\boldsymbol{\alpha}}=\prod_{j=0}^{n-1} w_{\alpha_{j}} .
$$

Because the unstable Jacobian is bounded below, $\varphi^{+}(\rho) \geq \Lambda^{+}>0$, the weights satisfy

$$
w_{a} \leq e^{-\Lambda^{+}}\|\alpha\|_{\infty}
$$

so after a some time the estimate (57) becomes sharper than the bound (56).

This type of estimate first appeared in the work of Anantharaman on Anosov flows [2]. It was extended to the case of scattering problems with a hyperbolic repeller in [57, Prop.6.3] (see also [51, Section 4]), and to situations with a nonconstant symbol $\alpha$ in [68].

8.1.3. Putting the pieces together. One applies the triangular inequality to get a bound on the sum of terms made up by $\mathcal{M}_{A_{1}}^{n}$ :

$$
\left\|\mathcal{M}_{A_{1}}^{n} u_{0}\right\| \lesssim \sum_{\boldsymbol{\alpha} \in A_{1}^{n} \text { admis. }} w_{\boldsymbol{\alpha}}=\sum_{a^{\prime}, a \in A_{1}}\left[\left(T^{w}\right)^{n}\right]_{a^{\prime} a},
$$

where we use the weighted transition matrix. For $n \gg 1$, the high power of the matrix $T^{w}$ is dominated by its Perron-Frobenius eigenvalue, which, according to (54), is close to the

\footnotetext{
${ }^{16}$ If the Lagrangian $\Lambda=\{(x, d S(x))\}$ for the generating function $S(x)$, a Lagrangian (or WKB) state associated with $\Lambda$ has the form $u_{0}(x)=f(x) e^{i S(x) / \hbar}$, with $f \in C_{c}^{\infty}$.
} 
topological pressure, so for some $\tilde{\epsilon}>0$ we get,

$$
\left\|\mathcal{M}_{A_{1}}^{n} u_{0}\right\| \leq C e^{n\left(\mathcal{P}\left(-\varphi^{+} / 2+\log |\alpha|\right)+\tilde{\epsilon}\right)} .
$$

So far we have considered the action of the propagator on very particular Lagrangian states $u_{0}$. However, any state $u$ microlocalized in $V_{\alpha_{0}}$ can be expanded into a "basis" $\left(u_{\zeta}\right)_{\zeta \in W}$ of such WKB states:

$$
u=\int_{W} \frac{d \zeta}{(2 \pi \hbar)^{d / 2}} \hat{u}(\zeta) u_{\zeta}+\mathcal{O}\left(\hbar^{\infty}\right)
$$

with $W$ a bounded domain in $\mathbb{R}^{d}$ and $\int_{W} d \zeta|\hat{u}(\zeta)|=\mathcal{O}(1)$. Applying $\mathcal{M}_{A_{1}}^{n}$ to the decomposition (59) and adding the contributions of the "tails" $\boldsymbol{\alpha}_{ \pm}$, we obtain for $n \gg 1$ the norm estimate

$$
\left\|\mathcal{M}(\hbar)^{n}\right\| \leq C \hbar^{-d / 2} e^{n\left(\mathcal{P}\left(-\varphi^{+} / 2+\log |\alpha|\right)+\tilde{\epsilon}\right)} .
$$

Crucially, the above estimate is valid for "large logarithmic times" $n \sim \tilde{\epsilon}^{-1} \log (1 / \hbar)$, for which we have $\hbar^{-d / 2} \leq e^{n \tilde{\epsilon}}$. We thus get

$$
\left\|\mathcal{M}(\hbar)^{n}\right\| \leq \exp \left\{n\left(\mathcal{P}\left(-\varphi^{+} / 2+\log |\alpha|\right)+2 \tilde{\epsilon}\right)\right\}
$$

which proves the spectral bound (50).

8.2. Is the pressure bound optimal? As shown above, the pressure bound (60) is obtained by evolving Lagrangian states $u_{0}$ through the components $\mathcal{M}_{\boldsymbol{\alpha}}$, resulting in the hyperbolic dispersive estimate (57), which is generally sharp. Then we applied the triangular inequality to bound the norm of $\mathcal{M}_{A_{1}}^{n} u_{0}$, and got the bound (58). The question is: how much does one "lose" through this triangular inequality?

The square norm of $\mathcal{M}_{A_{1}}^{n} u_{0}$ can be written as

$$
\left\langle\sum_{\boldsymbol{\alpha} \in A_{1}^{n} \text { admis. }} \mathcal{M}_{\boldsymbol{\alpha}} u_{0}, \sum_{\boldsymbol{\alpha} \in A_{1}^{n} \text { admis. }} \mathcal{M}_{\boldsymbol{\alpha}} u_{0}\right\rangle .
$$

If the states $\mathcal{M}_{\boldsymbol{\alpha}} u_{0}$ were orthogonal to each other, this scalar product would be given by a diagonal sum

$$
\sum_{\alpha \in A_{1}^{n} \text { admis. }}\left\|\mathcal{M}_{\boldsymbol{\alpha}} u_{0}\right\|^{2} \leq C \sum_{\boldsymbol{\alpha} \in A_{1}^{n} \text { admis. }} w_{\boldsymbol{\alpha}}^{2} \leq \exp \left\{n\left(\mathcal{P}\left(-\varphi^{+}+2 \log |\alpha|\right)+\tilde{\epsilon}\right)\right\} .
$$

The bound is sharper than (58), because for any nonzero test function $f$ one has $\mathcal{P}(2 f)<$ $2 \mathcal{P}(f)$. For instance, in the case of an open quantum map, $\alpha \equiv 1$ near $\mathcal{K}$, the pressure $\mathcal{P}\left(-\varphi^{+}\right)=-\gamma_{c l}$ is always negative, while $\mathcal{P}\left(-\varphi^{+} / 2\right)$ is negative only provided $\mathcal{K}$ is "thin enough".

Two states $\mathcal{M}_{\boldsymbol{\alpha}} u_{0}, \mathcal{M}_{\boldsymbol{\alpha}^{\prime}} u_{0}$ will indeed be (essentially) orthogonal if the final indices $\alpha_{n-1} \neq \alpha_{n-1}^{\prime}$ (the states are localized in disjoint sets), or if they are supported on Lagrangian leaves $\Lambda_{\boldsymbol{\alpha}}, \Lambda_{\boldsymbol{\alpha}^{\prime}} \subset V_{\alpha_{n-1}}$ at distance $\gg \hbar$ from one another: a nonstationary phase estimate then ensures that

$$
\left\langle\mathcal{M}_{\boldsymbol{\alpha}^{\prime}} u_{0}, \mathcal{M}_{\boldsymbol{\alpha}} u_{0}\right\rangle=\mathcal{O}\left(\hbar^{\infty}\right)
$$


This essential orthogonality indeed occurs for sequences of length $n \leq c \log (1 / \hbar)$, with $c>0$ sufficiently small. But for the large logarithmic times $n \sim \tilde{\epsilon}^{-1} \log (1 / \hbar)$ we need, many states $\mathcal{M}_{\boldsymbol{\alpha}} u_{0}$ will be supported by Lagrangians $\hbar$-close to one another, leading to nonnegligible off-diagonal terms

$$
\left\langle\mathcal{M}_{\boldsymbol{\alpha}} u_{0}, \mathcal{M}_{\boldsymbol{\alpha}^{\prime}} u_{0}\right\rangle \approx e^{i\left(\theta_{\boldsymbol{\alpha}}-\theta_{\boldsymbol{\alpha}^{\prime}}\right)} w_{\boldsymbol{\alpha}} w_{\boldsymbol{\alpha}^{\prime}} .
$$

The phases $\theta_{\boldsymbol{\alpha}}, \theta_{\boldsymbol{\alpha}^{\prime}}$ are the actions accumulated along the "paths" $\boldsymbol{\alpha}, \boldsymbol{\alpha}^{\prime}$; it is tempting to believe that these phases are pseudo-random. Namely, that they behave like independent random phases: the sum of the off-diagonal elements would then be of the same order as the sum (61) over diagonal terms, and lead to a spectral bound

$$
r_{s p}(\mathcal{M}(\alpha, \hbar)) \leq e^{\mathcal{P}\left(-\varphi^{+}+2 \log |\alpha|\right) / 2+\tilde{\epsilon}} .
$$

8.2.1. Phase cancellations in classical dynamics. Even if true, the pseudo-randomness of the phases $\theta_{\boldsymbol{\alpha}}$ seems very difficult to prove. What can be done rigorously? Partial phase cancellations were exhibited by Dolgopyat in his proof of exponential mixing for contact Anosov flows [24, 46]. In this situation the FIOs $\mathcal{M}(\alpha, \hbar)$ are replaced by Ruelle's transfer operator $\mathcal{L}_{s}$ associated with a certain expanding map $T$, defined on some unstable leaf $W^{+}$ by projecting $\kappa$ along the stable foliation:

$$
\mathcal{L}_{s} u(x) \stackrel{\text { def }}{=} \sum_{y: T(y)=x} e^{-s \tau(y)} u(y), \quad \text { so } \quad \mathcal{L}_{s}^{n} u(x) \stackrel{\text { def }}{=} \sum_{y: T^{n}(y)=x} e^{-s \tau_{n}(y)} u(y)
$$

Here $\tau$ is the Poincaré return time, and $\tau_{n}$ is the time accumulated after $n$ iterations, and the parameter $s=s_{0}+i t$, where the imaginary part $t$ should be compared with $\hbar^{-1}$. Using a nonintegrability property of the return time, Dolgopyat showed that for $t$ large enough partial phase cancellations occur in the above sum for $\mathcal{L}_{s}^{n} u$, leading to a shrinking of the spectral radius:

$$
\exists \epsilon_{0}>0, t_{0}>0, \forall t \geq t_{0}, \quad r_{s p}\left(\mathcal{L}_{s_{0}+i t}\right) \leq r_{s p}\left(\mathcal{L}_{s_{0}}\right) e^{-\epsilon_{0}} .
$$

Unfortunately, the improvement $\epsilon_{0}$ is hardly explicit.

A similar improvement was obtained in the case of the Laplacian on convex co-compact hyperbolic surfaces $X=\Gamma \backslash \mathbb{H}^{2}$ (see \$3.2). Following [39], let us define the essential spectral gap representing the optimal resonance free strip at high frequency:

$$
G(X) \stackrel{\text { def }}{=} \inf \left\{\sigma \leq n / 2, \operatorname{Res}\left(\Delta_{X}\right) \cap\{\operatorname{Re} s \geq \sigma\} \text { is finite }\right\} .
$$

Here the "pressure" bound (23) means that $G(X) \leq \delta$. Using the characterization of the resonances in terms of a certain Ruelle transfer operator $\mathcal{L}_{s}$, Naud [49] proved an improved spectral bound of the form (63) and showed that the pressure bound on $G(X)$ could be improved:

$$
\exists \epsilon_{1}>0, \quad G(X) \leq \delta-\epsilon_{1} .
$$

Jakobson and Naud further investigated the location of resonances for certain arithmetic convex co-compact surfaces, in both cases of "thick" $(\delta \in[1 / 2,1])$ and "thin" $(\delta \in(0,1 / 2))$ 
trapped sets [39]. Analyzing Selberg's zeta function, they managed to prove lower bounds for the essential spectral gap:

$$
\text { "thick" } K: \quad G(X) \geq \frac{\delta}{2}-\frac{1}{4}, \quad \text { "thin" } K: \quad G(X) \geq \frac{\delta(1-2 \delta)}{2} .
$$

They also conjecture that in both cases the essential spectral gap should be given by

$$
G(X)=\frac{\delta}{2}
$$

This conjecture is equivalent with the value $\mathcal{P}\left(-\varphi^{+}\right) / 2=-\gamma_{c l} / 2$ appearing in (62) (in the case $\alpha \equiv 1)$. Therefore, the conjecture (65) amounts to assume that the phases appearing in $\mathcal{L}_{s}^{n} u$ cancel each other at least as much as if they were random.

This conjecture is inspired by the case of arithmetic surfaces of finite volume (e.g. the modular group $\Gamma=S L(2, \mathbb{Z})$ ), for which the high frequency resonances are actually eigenvalues embedded in the absolute spectrum, with $\operatorname{Re} s_{j}=1 / 2=\delta / 2$.

A Dolgopyat type estimate (63) was also shown by Stoyanov in the case of classical scattering by $J$ convex obstacles in two dimensions [79] or higher dimensions [80] and also for more general Axiom A flows [81. In the case of scattering by convex obstables, there is no exact connection between the zeros of the semiclassical (Selberg-type) zeta function and the quantum resonances. Yet, Petkov and Stoyanov [63] were able to compare the long time quantum evolution $\mathcal{M}(k)^{n} u_{0}$ for some initial Lagrangian state $u_{0}$, with a (modifed) evolution of $u_{0}$ through a classical transfer operator of the form $\mathcal{L}_{s}, s=i k$. This connection allowed them to use the improved spectral gap for $\mathcal{L}_{s},|\operatorname{Im} s| \gg 1$, to (effectively) get a smaller spectral radius for $\mathcal{M}(k)$ than predicted in (50), hence a wider resonance free strip than predicted in Thm 1 .

How large could the resonance gap be for such obstacle problems? Could it be as large as $\gamma_{c l} / 2$, as conjectured above for hyperbolic surfaces? As noticed in \$6, the numerics performed for the 3 -disk scattering on $\mathbb{R}^{2}$ [47] shows a peak in the resonance density centered near $\operatorname{Im} k=-\gamma_{c l} / 2$. These numerics are unable to predict how the peak behaves in the high frequency limit. If this peak remains of positive width when $k \rightarrow \infty$, this would indicate that the resonance gap is smaller than $\gamma_{c l} / 2$.

It is very likely that this improvement on the resonance gap can be extended to the case of semiclassical Schrödinger operators (21) with hyperbolic repellers; the main difficulty probably resides in checking that the classical conditions for a Dolgopyat estimate to hold are met.

8.2.2. Some numerics for the open baker's map. Most numerical studies of open quantum maps were focussing on the spectral density and the fractal Weyl law, rather than the spectral radii. We have mentioned in $\$ 6.1$ that the numerics relative to several open maps show a peak in the radial spectral distribution near a value $r_{p e a k} \approx e^{-\gamma_{c l} / 2}$, which "pushes" 
the spectral radius to a larger value. Below we provide some numerical results for the open baker's map (see §6.1.1).

We only consider symmetric baker's map with $D$ symbols (that is the map (43) with $\left.x_{i}=i / D\right)$, so that the unstable Jacobian $\varphi^{+} \equiv \log D$. We let the hole consist in the union of $D-n$ Markov rectangles $(0<n<D)$. The topological pressure is then given by

$$
\mathcal{P}\left(-s \varphi^{+}\right)=\log n-s \log D .
$$

In particular, this pressure does not depend on which rectangles are removed, but only on their number. The baker's map is discontinuous along the boundaries of the rectangles, and these discontinuities are believed to induce diffraction effects at the quantum level; for this reason, the open baker's map does not satisfy the assumptions of Thm 9. Yet, if the leftmost and rightmost rectangles both belong to the hole, the trapped set $\mathcal{K}$ is at finite distance from the discontinuities, so it is reasonable to expect that the quantum spectrum should not be too sensitive to this diffraction. We have numerially computed the spectral radii of several open quantum baker's maps (see Fig. 14). The quantum dimension $N=(2 \pi \hbar)^{-1}$ was taken in a range $10 \lesssim N \lesssim 5000$. The first case (top left) is a baker with $D=5$ symbols, where the kept rectangles have indices $i=1,3$, so that the trapped set $\mathcal{K}$ is away from the boundaries. The spectral radii $r(N)$ seem to satisfy

$$
e^{\mathcal{P}\left(-\varphi^{+}\right) / 2}+\epsilon_{1}<r(N)<e^{\mathcal{P}\left(-\varphi^{+} / 2\right)}-\epsilon_{2},
$$

for some $\epsilon_{i}>0$, but keep fluctuating for large $N$. The parity of the eigenmodes (w.r.t. $x=0$ ) does not seem to play an important role.

On the opposite, in the case of the $D=5$ baker with kept rectangles $i=0,4$, the trapped set contains the axes $\{x=0\},\{\xi=0\}$, on which the map is discontinuous. The spectral radii of the even-parity sector satisfy the same bound as above, but the odd-parity spectral radius $r_{\text {odd }}(N) \approx e^{\mathcal{P}\left(-\varphi^{+} / 2\right)}$, thus barely violating the bound of Thm 9 . The reason why the spectral radius for the odd sector is larger than for the even one is unclear.

In the case $D=3$ (bottom), the trapped set also touches the discontinuity set; the value $e^{\mathcal{P}\left(-\varphi^{+} / 2\right)}>1$ is larger than the unitarity bound. As above, odd states show larger eigenvalues than even ones. The radii seem to satisfy

$$
e^{-\mathcal{P}\left(-\varphi^{+}\right) / 2}+\epsilon_{1}<r(N)<1-\epsilon_{2}, \quad \epsilon_{i}>0 \quad \text { fixed }
$$

indicating a gap below the unitarity bound.

On the figures we emphasize some geometric series $N=N_{o} D^{k}, k=0,1,2, \ldots$, because such series were shown important when studying the fractal Weyl law [55, 56]. The spectral radii along such series indeed show some regularity, especially when taking the parity of $k$ into account.

In the case of the Walsh quantization of the symmetric $D$-baker's map presented in $\$ 6.1 .2$, the spectral radius of $M_{N}$ (for $N=D^{k}, k \in \mathbb{N}$ ) is given by the largest eigenvalue $\lambda$ of the $n \times n$ matrix $\widetilde{\Omega}_{D}$, obtained by removing from the $D$-discrete Fourier transform the 
Quantum open baker $(\mathrm{D}=5$, columns 1-3)

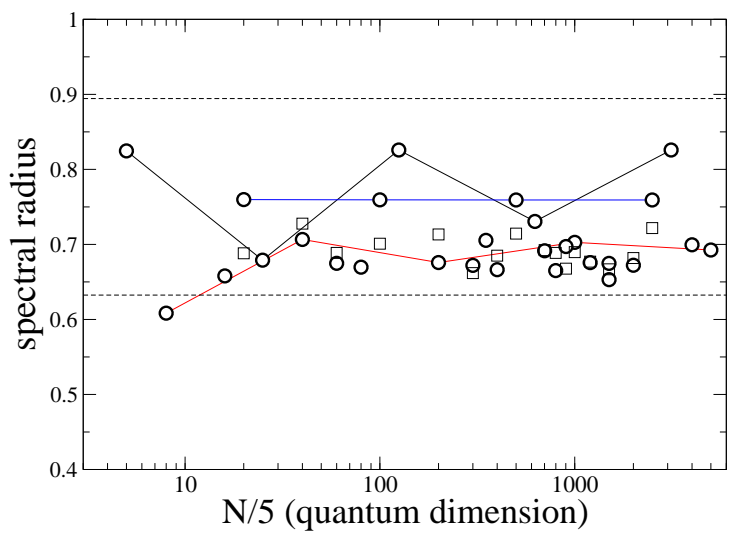

Quantum open baker ( $\mathrm{D}=5$, columns $0-4)$

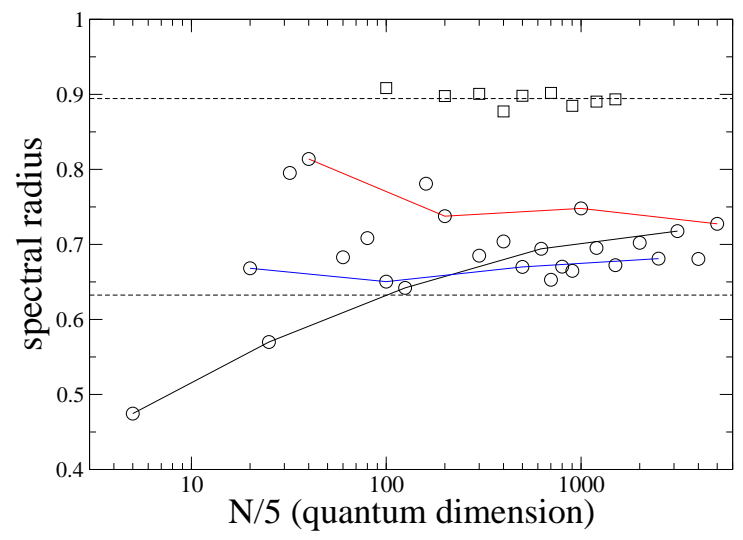

Quantum open baker ( $\mathrm{D}=3$, columns 0-2)

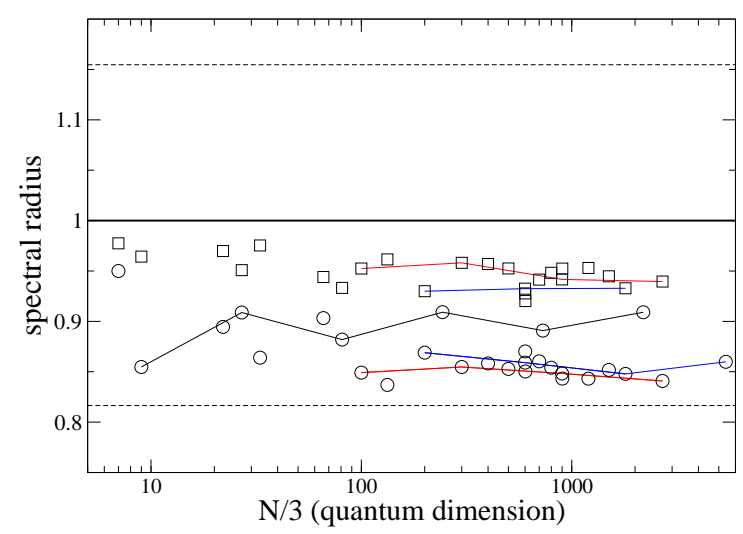

FIGURE 14. Spectral radii of various quantum symmetric open baker's maps, with $n=2$ kept rectangles. $N=(2 \pi \hbar)^{-1}$ is the quantum dimension. Eigenmodes are split according to parity: even (o) vs. odd ( $\square$ ). Straight lines emphasize geometric series $N=N_{o} D^{k}, k=0,1,2, \ldots$ Top left: $D=5$, kept rectangles $i=1,3$. Top right: $D=5$, kept rectangles $i=0,4$. Bottom: $D=3$, kept rectangle $i=0,2$. The dashed horizontal lines indicate the values $e^{\mathcal{P}\left(-\varphi^{+} / 2\right)}>e^{\mathcal{P}\left(-\varphi^{+}\right) / 2}$.

$D-n$ columns and lines corresponding to the hole. As a result, the spectral radius of $M_{N}$ is the same for all quantum dimension $N=D^{k}$. The pressure bound

$$
r_{s p}\left(M_{N}\right) \leq e^{\mathcal{P}\left(-\varphi^{+} / 2\right)}=\frac{n}{\sqrt{D}}
$$

obviously results from the fact that all entries of $\widetilde{\Omega}_{D}$ have modulus $\frac{1}{\sqrt{D}}$. The various examples we have treated in [55, 56] show that for this model the spectral radius is unrelated 
with the value $e^{-\gamma_{c l} / 2}=\sqrt{\frac{n}{D}}$, its values can vary across the full interval $\left[0, \min \left(1, \frac{n}{\sqrt{D}}\right)\right]$ (including the extremal values), depending on the explicit phases in the matrix $\widetilde{\Omega}_{D}$. This situation is far from generic, and seems to rely on the fact that the underlying harmonic analysis is associated with the Walsh-Fourier transform.

A preliminary investigation of a smoothed version of the (standard) quantum baker's map apparently leads to a Dolgopyat type partial phase cancellation, which would then force the spectral radius to be $\leq \frac{n}{\sqrt{D}}-\epsilon_{2}$ for some $\epsilon_{2}>0$. Yet, in spite of the explicit (and relatively simple) expressions for the the phases, it seems impossible to push these cancellations such as to recover the conjectured "optimal" bound $\sqrt{\frac{n}{D}}$.

\section{Phase space structure of havefunctions}

In this last section we address Question (3), that is we investigate the structure of the "eigenfunctions" (in a generalized sense) of our scattering system introduced in $\$ 1.1$.

The first class of such eigenfunctions will be the metastable states $u_{j}(\hbar)$ associated with the (discrete) resonances $z_{j}(\hbar)$. They satisfy the differential equation $\left(P(\hbar)-z_{j}\right) u_{j}=0$, are purey outgoing and blow up exponentially at infinity.

On the opposite, for any real energy $E$ the scattering functions form an infinite dimensional space of functions satisfying $(P(\hbar)-E) u=0$. They are not square-integrable either, but contain both incoming and outgoing components.

In both cases, we will focus on the structure of these functions in the interaction region, say the ball $B\left(0, R_{0}\right)$.

9.1. Metastable states. Let $u_{j}(\hbar)$ be the metastable state associated with a resonance $z_{j}(\hbar)$ of our scattering Hamiltonian $P(\hbar)$. We may (somewhat arbitrarily) normalize this state inside the interaction region $B\left(R_{0}\right)$, by putting

$$
\left\|u_{j}(\hbar)\right\|_{L^{2}\left(B\left(R_{0}\right)\right)}=1 \text {. }
$$

In order to connect oneself with the classical dynamics, it is natural to analyze the modes $u_{j}(\hbar)$ in terms of their associated phase space distributions. Let us recall that to any function $u \in L^{2}$ one can associate its Wigner function $W_{u}^{\hbar}(x, \xi)$, depending quadracally on $u$ (the formula is given in (70) ). This Wigner function (or the Husimi function obtained by a smoothing on the scale $\sqrt{\hbar}$ ) is interpreted $\sqrt{17}$ as a phase space probability density for the state $u$. The distribution $W_{u}^{\hbar}=W_{u}^{\hbar}(x, \xi) d x d \xi$ will be called the Wigner distribution (or signed measure).

Describing the individual functions $W_{u_{j}(\hbar)}^{\hbar}$ seems a hopeless task. On the other hand, it is often possible to derive some asymptotic properties of a semiclassical sequence of such functions.

\footnotetext{
${ }^{17}$ The Wigner function generally takes both positive and negative values, which makes this interpretation a bit questionable. On the opposite, the Husimi function is nonnegative.
} 
Take any sequence $\hbar_{k} \rightarrow 0$, and for each $\hbar=\hbar_{k}$ choose some resonance $z(\hbar)=z_{j}(\hbar) \in$ $D(E, C \hbar)$, a corresponding metastable state $u(\hbar)=u_{j}(\hbar)$, and construct its Wigner distribution $W_{u(\hbar)}^{\hbar}$. We will then be interested in the asymptotic behaviour of the sequence $\left(W_{u(\hbar)}^{\hbar}\right)_{\hbar=\hbar_{k}}$ when $\hbar \rightarrow 0$.

Bony and Michel [7, Thm 2.1] showed that, for a general trapping potential, the Wigner distributions $W_{u(\hbar)}$ are semiclassically negligible away from the outgoing tail $K_{E}^{+}$: for any test function $a \in C_{c}^{\infty}\left(T^{*} X\right)$ supported away from $K_{E}^{+}$, one has

$$
\left\langle W_{u(\hbar)}^{\hbar}, a\right\rangle=\mathcal{O}\left(\hbar^{\infty}\right) \text {. }
$$

This estimate does not depend on the structure of the flow on $K_{E}$.

The same type of result was also obtained in the case of the open baker's map [42, 52, where some Husimi measures were numerically computed, and shown to concentrate on the outgoing tail $\mathcal{K}^{+}$(see this set on Fig. 11, center, and compare with Fig. 15).

A more precise asymptotic description of the Wigner (or Husimi) distributions is provided by the concept of semiclassical measure, that is a measure $\mu$ on phase space 18 , obtained as a limit (in the weak-* topology) of the sequence of distributions $\left(W_{u(\hbar)}^{\hbar}\right)_{\hbar \rightarrow 0}$, equivalently the limit of certain extracted subsequence $\left(W_{u(\hbar)}^{\hbar}\right)_{\hbar \in S}\left(S\right.$ is some infinite subsequence of $\left.\left(\hbar_{k}\right)\right)$. This measure describes the asymptotic phase space distribution of the metastable states along the subsequence $(u(\hbar))_{\hbar \in S}$. A priori, several limit measures $\mu$ may be extracted from the original sequence, corresponding to different subsequences $S$. Semiclassical measures
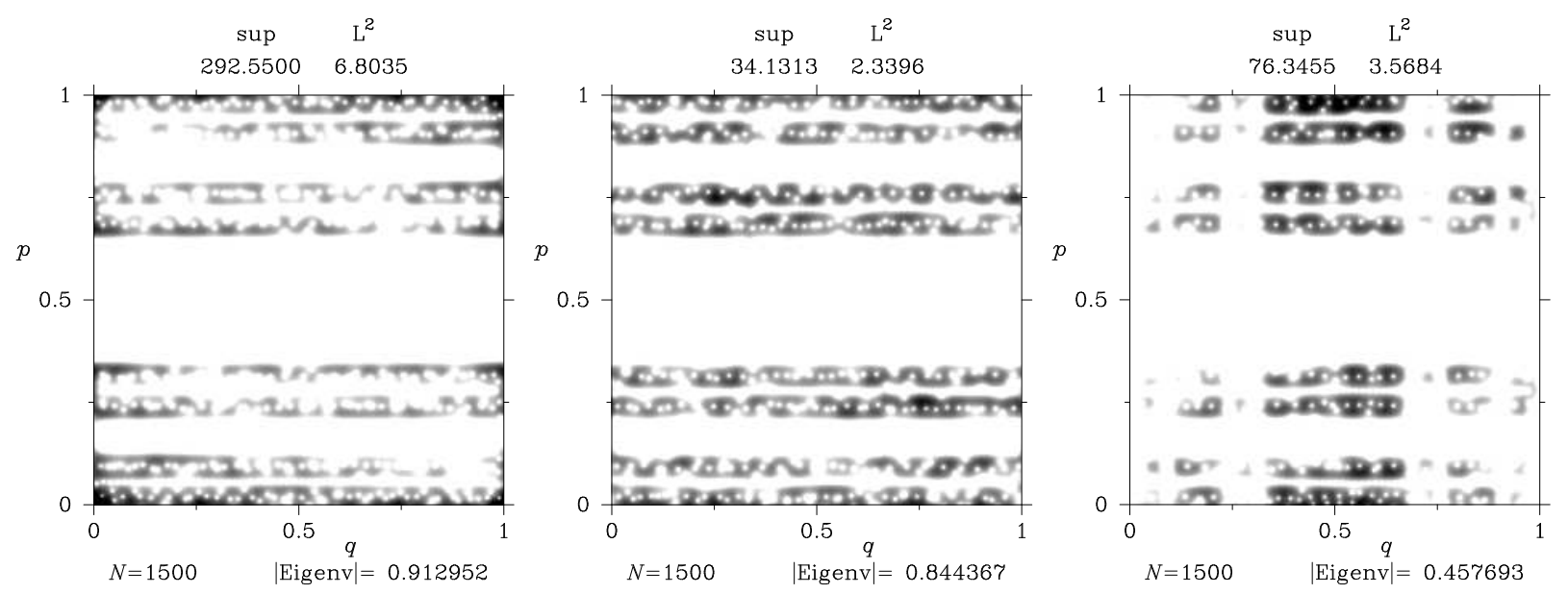

FigurE 15. Husimi functions of three metastable states of the quantum symmetric open 3-baker (logarithmic grey scale). The high intensities (black) are clearly localized on $\mathcal{K}^{+}$.

were investigated in the case of closed chaotic system (say, the geodesic flow on a compact

\footnotetext{
${ }^{18} \mathrm{We}$ will only consider the restriction of this measure on the interaction region $T^{*} B\left(0, R_{0}\right)$.
} 
Riemannian manifold of negative curvature). Any semiclassical measure associated with the eigenstates of $P(\hbar)=-\hbar^{2} \Delta_{X} / 2$ must be invariant w.r.t. the classical flow. Furthermore, the quantum ergodicity theorem states that, as long as the flow is ergodic w.r.t. the Liouville measure, then one can extract a subsequence $S$ of density one 19 , such that $\left(W_{u(\hbar)}^{\hbar}\right)_{\hbar \in S}$ converges to the Liouville measure on $p^{-1}(1 / 2)$ [70, 90, 19].

In the frameworks of potential scattering [57] or open chaotic maps [42, 52, a generalization of the above invariance property was obtained for semiclassical measures associated with sequences of metastable states.

Theorem 10. [57] Consider a scattering Hamiltonian (21) such that for some $E>0$ the trapped set $K_{E}$ is a hyperbolic repeller. Take a sequence of resonances $(z(\hbar) \in D(E, C \hbar))_{\hbar \rightarrow 0}$, and extract a subsequence $\left(W_{u(\hbar)}^{\hbar}\right)_{\hbar \in S}$ converging to a semiclassical measure $\mu$ on $T^{*} B\left(0, R_{0}\right)$.

Then $\mu$ will be invariant up to a decay rate $\Lambda \geq 0$ :

$$
\forall t \geq 0, \quad \Phi^{t *} \mu=e^{-t \Lambda} \mu \quad \text { inside the interaction region } T^{*} B\left(0, R_{0}\right) .
$$

Furthermore, the subsequence $S$ must be such that the resonances $(z(\hbar))_{\hbar \in S}$ satisfy

$$
\lim _{\hbar \in S, \hbar \rightarrow 0} \frac{\operatorname{Im} z(\hbar)}{\hbar}=-\frac{\Lambda}{2} .
$$

Any measure satisfying (66) (at least inside the interation region) will be called a $\Lambda$ eigenmeasure for the flow. In the case of an open map, a $\Lambda$-eigenmeasure is characterized by the property

$$
\kappa^{*} \mu=e^{-\Lambda} \mu .
$$

$\Lambda$-eigenmeasures are easy to classify. For instance, in the case of open maps, each $\Lambda$ eigenmeasure is uniquely determined by its restriction on $\mathcal{K}^{+} \cap \kappa(V) \backslash V$, which can be arbitrary. All such measures are supported on $\mathcal{K}^{+}$, and satisfy $\mu(\mathcal{K})=0$ for $\Lambda>0$, while they are supported on $\mathcal{K}$ iff $\Lambda=0$.

The above theorem shows that any semiclassical measure is necessarily a $\Lambda$-eigenmeasure, with decay rate $\Lambda$ equal to the asymptotic quantum decay rates. In view of the quantum ergodicity result for chaotic closed systems, the following question naturally arises:

Given $\Lambda \geq 0$, and considering a sequence of resonances $(z(\hbar))_{\hbar \in S}$ satisfying (67), which $\Lambda$-eigenmeasures can be obtained as semiclassical measures? Is there a "favoured" limit, or even a unique one?

This question presumes that there exist sequences of resonances satisfying (67), a fact which depends on the semiclassical distribution of resonances; in case the strong form (41) of fractal Weyl law holds, such a sequence exists if the profile function satisfies $\frac{d F}{d \gamma}(\Lambda / 2)>0$.

We have noticed before that, according to several numerical results, the density of resonances often shows a peak near the value $\Lambda=\gamma_{c l}$. For this specific value of $\Lambda$, there exists

\footnotetext{
${ }^{19}$ This means that this sequence contains "almost all" the eigenstates.
} 
a "natural" $\Lambda$-measure, which is obtained by iterating an initial smooth measure $\mu_{0}$ (with support intersecting $\mathcal{K}^{-}$):

$$
\mu_{\text {nat }}=\lim _{t \rightarrow \infty} \mathcal{N}_{t} \Phi^{t *} \mu_{0}, \quad \text { respectively } \quad \mu_{\text {nat }}=\lim _{n \rightarrow \infty} \mathcal{N}_{n} \kappa^{n *} \mu_{0},
$$

with $\mathcal{N}_{t}, \mathcal{N}_{n}$ appropriate normalization factors (see (17]) ). Yet, the study of [52] did not reveal that this measure played any particular role for the open quantum baker's map.

In 42 the authors computed averages of the spatial densities $\left|u_{j}(x)\right|^{2}$ over a few eigenstates with comparable decay rates, for the symmetric open 3-baker. They noticed strong self-similar properties of the densities, depending on the decay rates. Some of the individual Husimi functions of [52] were also featuring a selfsimilar behaviour in both the momentum and position directions.

Rigorous results were obtained in the case of the Walsh-quantized open baker's map [40, 52], using explicit formulas for the eigenstates. In this model most eigenvalues $\lambda_{j}(\hbar)$ have large multiplicities, leaving a lot of freedom to construct eigenstates. In [52, 40] it was shown that, for the Walsh-quantized symmetric 3-baker, any semiclassical sequence of eigenstates $(u(\hbar))_{\hbar \rightarrow 0}$ with eigenvalues converging towards the outer circle $|\lambda(\hbar)| \rightarrow r_{\max }$ (resp. the inner circle $|\lambda(\hbar)| \rightarrow r_{\min }$ ) of the nontrivial spectrum, converges to a single semiclassical measure $\mu_{\max }$ (resp. $\mu_{\min }$ ), which is of Bernoulli type, therefore perfectly selfsimilar. This is a form of "quantum unique ergodicity" at the edges of the nontrivial spectrum. On the opposite, for any value $r \in\left(r_{\min }, r_{\max }\right)$, we exhibited many semiclassical measures associated with sequences $(u(\hbar))_{\hbar \rightarrow 0}$ of asymptotic decay rates $|\lambda(\hbar)| \rightarrow r$. For $r=e^{-\gamma_{c l} / 2}$ we showed that the natural measure $\mu_{\text {nat }}$ is not a semiclassical measure.

9.2. Scattering states. Metastable states appear in expansions of the resolvent of $P(\hbar)$, and consequently in expansions for the time dynamics [84, 14]. Another class of generalized eigenstates is more natural from the point of view of scattering theory, namely the scattering states, used to define the scattering matrix (see \$1.1). In the semiclassical setting of a scattering Hamiltonian $P(\hbar)$ on a manifold $X$, a scattering state at energy $E>0$ is a wavefunction $u_{E}=u_{E}(\hbar)$ satisfying the differential equation $(P(\hbar)-E) u_{E}=0$, and satisfying certain conditions at infinity.

If $X \equiv \mathbb{R}^{d}$ outside the interaction region $B\left(0, R_{0}\right)$, one can expand $u_{E}(x)$ using a basis of incoming and outgoing waves, as in Eqs. (4).5). Fixing the incoming part of $u_{E, \text { in }}$ near infinity uniquely determines the full wavefunction $u_{E}$, and in particular determines its outgoing part $u_{E, \text { out }}$, the relation between $u_{E, \text { in }}$ and $u_{E, \text { out }}$ defining the scattering matrix $S(E)$. We ask the following question:

Given $u_{E, i n}$, what is the spatial (or phase space) structure of $u_{E}$ inside the interaction region?

In the semiclassical/high-frequency limit, the usual basis states for the incoming wave $u_{E, \text { in }}$ (namely the angular momentum eigenstates, see (5)) are Lagrangian states associated with certain Lagrangian submanifolds of the energy shell, for instance a spherically 
symmetric incoming wave sits on the Lagrangian manifold $\{(x, \xi=-\sqrt{2 E} x /|x|)\}$. Most of the trajectories on this manifold will be scattered inside the interaction region and then exit it towards infinity after a short transient time. Still, a small fraction of the incoming trajectories may be trapped during a long time in this region, travelling close close to $K_{E}$, or even be trapped for ever if they exactly belong to the incoming tail $K_{E}^{-}$. How do these trapped (or long transient) trajectories influence the structure of $u_{E}$ ?
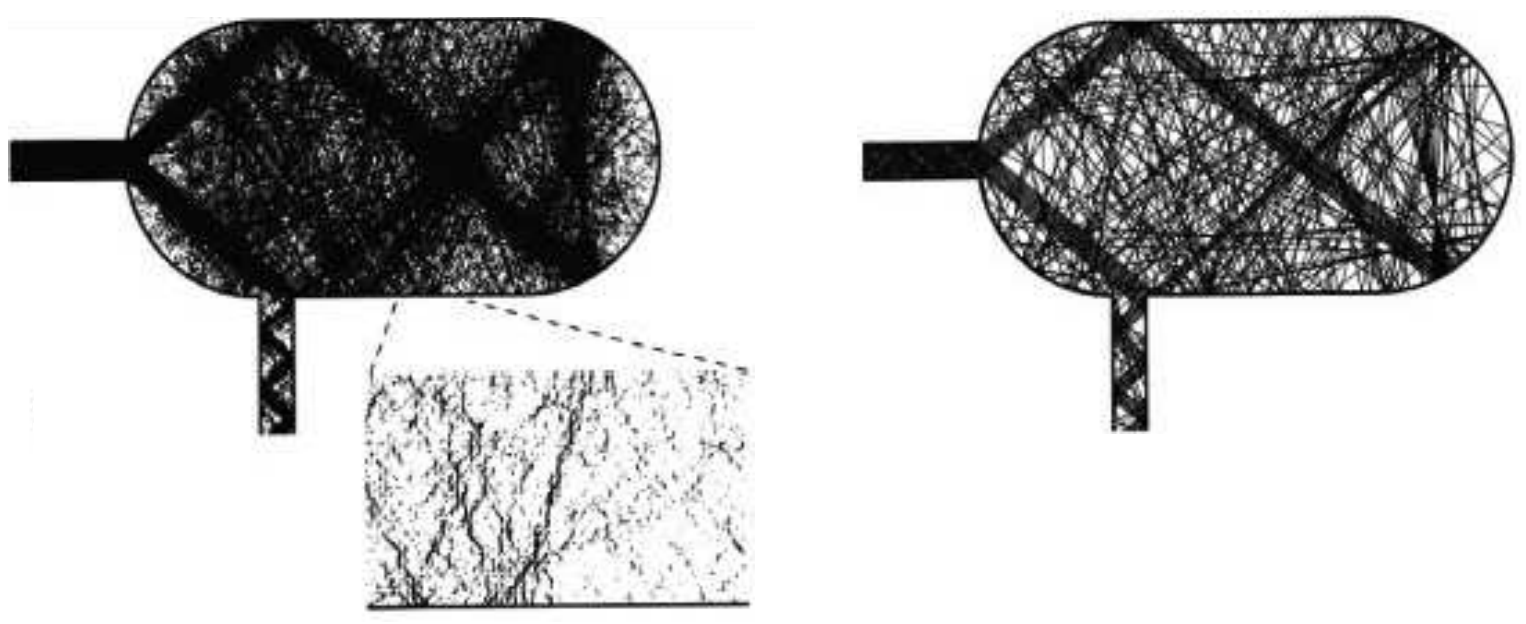

FIgURE 16. Left: density plot of a scattering state $\left|u_{E}(x)\right|^{2}$, with incoming part $u_{E, \text { in }}$ a plane wave in the left lead into the stadiumshaped cavity. Right: corresponding classical trajectories incoming from the left opening with angles $\pm \theta_{n}$. Reprinted with permission from H. Ishio and J.P. Keating, J. Phys. A 37 (2004) L217-L223. Copyright 2004 by the Institute of Physics.

This question has been studied numerically by Ishio and Keating [38] in a different geometry, namely the case of a $2 \mathrm{~d}$ chaotic cavity opened by two infinite "leads" (waveguides). In this case, the incoming wave $u_{E, \text { in }}$ is given by plane waves inside the left lead, $u_{E}(x, y)=\sin \left(k_{n} y\right) e^{i k_{l} x}$, where the longitudinal and transverse wavevectors $k_{l}, k_{n}$ satisfy

$$
k_{n}=\pi n / L, \quad E=\frac{\hbar^{2}}{2}\left(k_{l}^{2}+k_{n}^{2}\right), \quad n \in \mathbb{N} \backslash 0, \quad L \text { the width of the lead. }
$$

This incoming wave semiclassically corresponds to a pencil of trajectories coming out of the lead with an angle $\pm \theta_{n}, \theta_{n}=\arcsin \left(k_{n} / k_{l}\right)$. Two scattering states with such incoming components were numerically computed in [38], one of them is shown on the left of Fig. 16. In both cases the density $\left|u_{E}(x)\right|^{2}$ is strongly imprinted by short transient orbits. The authors also derived an approximate semiclassical expression for $u_{E}(x)$, as a sum over classical trajectories, and showed that this expression is quite accurate for the two examples of states they have computed. They distinguished between two complementary situations: 
for weakly open situations, the contributions of long trajectories is important (even divergent); on the opposite, for very open systems, the contribution of long trajectories decays exponentially fast, so that the wavefunction is mainly influenced by the short transient trajectories. This dichotomy is of course reminiscent of the one mentioned in $\$ 1.2$.

More recently, Guillarmou and Naud [33] studied the scattering states for convex cocompact manifolds $X=\Gamma \backslash \mathbb{H}^{n+1}$, also called the Eisenstein functions in this context (the spectral parameter is $s=n / 2+i t, t \gg 1)$. A convenient "basis" consists in the functions $u_{s, y}(x)$ which become singular when $x$ converges to a given point $y$ of the boundary $\partial X$ : the incoming wave is then associated with the unstable manifold made of the geodesics issued from $y$. In such a homogeneous situation, the wavefunction $u_{s, y}(x)$ can be simply expressed by a sum over the group $\Gamma$. The authors are able to precisely describe $u_{s, y}$ provided the trapped set is "thin", that is the dimension of the limit set satisfies $\delta<n / 2$ (equivalently, $\left.\mathcal{P}\left(-\varphi^{+} / 2\right)<0\right)$. This is a precise criterion for the "very open" situation of [38]. One can then compute the semiclassical measure associated with the family $\left(u_{n / 2+i t, y}\right)_{t \rightarrow \infty}$ : it is an invariant measure supported by the full unstable manifold issued from $y$.

If one averages the densities $\left|u_{s, y}(x)\right|^{2}$ over the boundary point $y$, one recovers the uniform (Haar) measure on $X$, plus a semiclassically small correction given by a sum over periodic orbits, similarly with Gutzwiller's trace formula for closed systems (one difference being that the sum over the orbits is absolutely convergent).

This description of scattering states can certainly be extended to more general geometries or systems with a "thin" hyperbolic trapped set.

\section{Conclusion}

We have presented several analytical methods used to analyze the spectral properties of scattering operators in the semiclassical/high frequency limit, in cases where the set of classically trapped trajectories is a hyperbolic repeller. In particular, the number of longliving resonances near some classical energy $E>0$ was bounded from above by a fractal power of the semiclassical parameter, reflecting the fact that these long living states must be supported on the trapped set, which is a fractal subset of the energy shell. We stated two types of "fractal Weyl law" conjectures, predicting that this upper bound should be sharp, and presented some numerical results in favor of these laws, both for scattering flows and for the model of open quantum maps.

A second result is the presence of a "resonance gap" (or a uniform lower bound for the quantum decay rates), provided the instability of the flow exceeds its complexity (precisely, provided the topological pressure $\mathcal{P}\left(-\varphi^{+} / 2\right)$ is negative). This criterion allows to split such chaotic scattering systems between "very open" vs. "weakly open" systems. We showed that this dichotomy was also relevant in the precise description of scattering wavefunctions.

At the technical level, we presented quantum monodromy operators associated with a quantum scattering flow, which can be used to investigate this spectral problem. These 
operators, which contain the full long living quantum dynamics, can be deformed such as to live in a "minimal" neighbourhood of the trapped set, still faithfully representing the "quantum mechanics on the trapped set". They resemble Ruelle transfer operators appearing in classical dynamics. Hopefully, a more precise analysis of these operators could deliver some nontrivial information on the resonance spectrum, like a proof of the fractal Weyl law (under some genericity assumption) or a sharper criterion for a resonance free strip.

The resonances were analyzed as eigenvalues of certain nonselfadjoint pseudodifferential operators. The techniques presented above can also be used in a different context, namely the study of a "closed" quantized chaotic systems in the presence of some "damping", e.g. the case of damped waves propagating on a manifold of negative curvature. In that case there is no "escape to infinity", but the high frequency spectral problem presents similar features [75]. For instance, fractal Weyl upper bounds were obtained for such systems [3], and a bound for the decay rates in terms of a topological pressure was also proved in this context [68, 69, with applications to the stabilization of the damped waves.

The same type of ideas could also be useful when describing the scattering by a dielectric cavity, relevant in the description of quasi-2d microlasers (see e.g. [88, 73, 6] and references therein). In such situations, the damping is due to the fact that a wavepacket propagating inside the cavity loses a fraction of its energy when being reflected by the boundary of the cavity, the rest of the energy being refracted outside to infinity. How does the shape of the cavity influence the resonance spectrum, in particular in case the internal dynamics is chaotic? How do the metastable states look like? To my knowledge, the rigorous studies of such cavities have so far been restricted to strictly convex cavities with smooth boundaries [15], for which the ray dynamics cannot be purely chaotic.

\section{Appendix A. A Brief Review of $\hbar$-PSEUdodifferential CAlCulus}

We recall some definition and basic properties of Weyl's quantization, in the semiclassical setting. For simplicity, we will only consider operators on the Euclidean space $\mathbb{R}^{d}$. See [23, Chapter 7] for a detailed discussion of semiclassical quantization, [27, Chap.4, Part 3] for the pseudodifferential calculus for the symbol classes presented below, and [27, Chap.13] for its generalization to the calculus on manifolds.

A.1. Weyl quantization and pseudodifferential calculus. Weyl's quantization associates to a smooth phase space function $a \in C^{\infty}\left(T^{*} \mathbb{R}^{d}\right)$ (the classical observable, or symbol) an operator acting on $u \in C_{c}^{\infty}\left(\mathbb{R}^{d}\right)$ as follows:

$$
\begin{aligned}
{\left[a^{w} u\right](x) } & =\left[\mathrm{Op}_{\hbar}(a) u\right](x) \\
& \stackrel{\text { def }}{=} \frac{1}{(2 \pi \hbar)^{d}} \iint a\left(\frac{x+y}{2}, \xi\right) e^{i\langle x-y, \xi\rangle / \hbar} u(y) d y d \xi .
\end{aligned}
$$


In these notations, $\hbar \in(0,1]$ is Planck's "constant" (which we always assume to be "small"). The integral converges absolutely only if $a(x, \xi)$ decays fast enough w.r.t. $\xi$, but by integrating by parts one can easily extend the definition to functions growing algebraically in $\xi$. The classes of symbols presented below are engineered such that the above formula makes sense.

Weyl's quantization leads to the definition of the Wigner distribution $W_{u}^{\hbar}$ associated with a function $u \in L^{2}$, by the following duality:

$$
\forall a \in C_{c}^{\infty}\left(T^{*} \mathbb{R}^{d}\right), \quad\left\langle W_{u}^{\hbar}, a\right\rangle_{\left(C_{c}^{\infty}\right)^{\prime}, C_{c}^{\infty}} \stackrel{\text { def }}{=}\left\langle u, \mathrm{Op}_{\hbar}(a) u\right\rangle_{L^{2}}
$$

When $\hbar$ is small, the product of two operators $\mathrm{Op}_{\hbar}(a) \mathrm{Op}_{\hbar}(b)$ can be analyzed through their symbols $a, b$. That product is itself an operator of the form $\mathrm{Op}_{\hbar}(c)$, with a symbol $c(x, \xi)$ given by the Moyal product of $a$ and $b$ :

$$
\begin{aligned}
c & =a \sharp_{\hbar} b \stackrel{\text { def }}{=} a \exp \left(\frac{i h}{2}\left(\left\langle\overleftarrow{D}_{\xi}, \vec{D}_{x}\right\rangle-\left\langle\overleftarrow{D}_{x}, \vec{D}_{\xi}\right\rangle\right)\right) b \\
& \sim a b+\frac{i \hbar}{2}\{a, b\}+\sum_{j \geq 2} \frac{(i \hbar / 2)^{j}}{j !} a\left(\left\langle\overleftarrow{D}_{\xi}, \vec{D}_{x}\right\rangle-\left\langle\overleftarrow{D}_{x}, \vec{D}_{\xi}\right\rangle\right)^{j} b
\end{aligned}
$$

where $D_{\bullet}=-i \partial_{\bullet}$, and $\{a, b\}$ is the Poisson bracket. The above sum is a good asymptotic expansion when $\hbar \rightarrow 0$, in the sense that the sum up to the term $j=N-1$ gives a good approximation of $c$, with a remainder $\mathcal{O}\left(\hbar^{N}\right)$. It is at the heart of pseudodifferential calculus. Even if $a, b$ are independent of $\hbar$, the symbol $c$ does depend on $\hbar$. It thus makes sense to define classes of $\hbar$-dependent symbols, characterized by the regularity property of $a(x, \xi ; \hbar)$, uniformly in the limit $\hbar \rightarrow 0$.

One standard class of symbols is the following: for $k \in \mathbb{R}$, let

$$
\begin{array}{r}
S^{k}\left(T^{*} \mathbb{R}^{d}\right)=\left\{a \in C^{\infty}\left(T^{*} \mathbb{R}_{x, \xi}^{d} \times(0,1]_{\hbar}\right): \forall \alpha, \beta \in \mathbb{N}^{d},\right. \\
\left.\left|\partial_{x}^{\alpha} \partial_{\xi}^{\beta} a(x, \xi ; \hbar)\right| \leq C_{\alpha, \beta}(1+|\xi|)^{k-|\beta|}\right\},
\end{array}
$$

The improved decay in $\xi$ upon differentiation is necessary for the class to be invariant upon a smooth change of coordinates, which is crucial when extending the formalism to manifolds. The corresponding operator classes are denoted by $\Psi^{k}\left(\mathbb{R}^{d}\right)$. For instance, the Schrödinger operator (21) is the Weyl quantization of the symbol $p(x, \xi)=\frac{|\xi|^{2}}{2}+V(x) \in S^{2}\left(T^{*} \mathbb{R}^{d}\right)$.

These symbol classes are closed under composition: for $a \in S^{k}, b \in S^{\ell}$, the product operator $\mathrm{Op}_{\hbar}(a) \mathrm{Op}_{\hbar}(b)=\mathrm{Op}_{\hbar}(c)$ belongs to $\Psi^{k+\ell}$. An important property is the action on $L^{2}\left(\mathbb{R}^{d}\right)$. For $a \in S^{0}\left(T^{*} \mathbb{R}^{d}\right)$, the operators $\mathrm{Op}_{\hbar}(a)$ are bounded on $L^{2}\left(\mathbb{R}^{d}\right)$, with

$$
\left\|\mathrm{Op}_{\hbar}(a)\right\|_{L^{2} \rightarrow L^{2}}=\|a(\hbar)\|_{L^{\infty}}+\mathcal{O}(\hbar) .
$$

If $a(x, \xi)$ is real valued, $\mathrm{Op}_{\hbar}(a)$ will be self-adjoint on $L^{2}$. In this case, one can also analyze functions of $\mathrm{Op}_{\hbar}(a)$ using their symbols: for a smooth function $f: \mathbb{R} \rightarrow \mathbb{R}$, the operator $f\left(\mathrm{Op}_{\hbar}(a)\right)$ belongs to $\Psi^{0}\left(\mathbb{R}^{d}\right)$, with symbol $f(a)+\mathcal{O}(\hbar)$. For instance, in $\$ 4.2$ the operators 
$e^{ \pm t G^{w}}$ belong to $\Psi^{0}\left(\mathbb{R}^{d}\right)$, and the composition rule (71) shows that the conjugated operator $e^{-t G^{w}} P_{\theta}(\hbar) e^{t G^{w}}$ belongs to $\Psi^{2}\left(\mathbb{R}^{d}\right)$, with a symbol of the form (27).

A.2. Exotic symbol classes. The symbols $a \in S^{0}\left(T^{*} \mathbb{R}^{d}\right)$ fluctuate on distances $\sim 1$. For our purposes, we also needed to consider symbols fluctuating on microscopic distances. For $k \in \mathbb{R}$ and $\delta \in[0,1 / 2]$, we consider the "exotic" symbol classes

$$
\begin{aligned}
S_{\delta}^{k}\left(T^{*} \mathbb{R}^{d}\right)=\{ & a \in C^{\infty}\left(T^{*} \mathbb{R}^{d} \times(0,1]\right): \forall \alpha, \beta \in \mathbb{N}^{d}, \\
& \left.\left|\partial_{x}^{\alpha} \partial_{\xi}^{\beta} a(x, \xi ; h)\right| \leq C_{\alpha, \beta} \hbar^{-\delta(|\alpha|+|\beta|)}(1+|\xi|)^{k-|\beta|}\right\},
\end{aligned}
$$

which encompasses symbols fluctuating on distances $\gtrsim \hbar^{\delta}$. For $\delta<1 / 2$, the expansion (71) makes sense, and we can still use the symbol to analyze the operator. These exotic classes were used to construct the exponential weights of $\$ 4.2$. If we take $\varepsilon=\hbar^{\delta}$, the escape function $G(x, \xi)$ must belong to the class $\log (1 / \hbar) S_{\delta}^{0}\left(T^{*} X\right)$ (see the model function $G_{1}(x, \xi)$ of (31) $)$, and the corresponding functional calculus allows to analyze the operators $e^{ \pm t G^{w}}$ and $e^{-t G^{w}} P(\hbar) e^{t G^{w}} \in \Psi_{\delta}^{2}\left(\mathbb{R}^{d}\right)$. The symbol $\alpha$ of (35) also belongs to an exotic class $S_{\delta}^{0}$.

A.3. Fourier integral operators. A time dependent Hamiltonian $p(t, x, \xi) \in C\left([0,1]_{t}, S^{2}\left(T^{*} \mathbb{R}^{d}\right)\right)$ generates a nonautonomous symplectic flow $\left(\kappa_{t}\right)_{t \in[0,1]}$ through Hamilton's equations

$$
\frac{d \kappa_{t}}{d t}=\left(\kappa_{t}\right)_{*} H_{p(t)}, \quad \kappa_{0}=I d, \quad t \in[0,1] .
$$

Then, the family of unitary operators $U(t)$ defined by

$$
i \hbar \partial_{t} U(t)=U(t) p^{w}(t), \quad U(0)=I d,
$$

defines a family of quantum propagators, which are unitary Fourier Integral Operators (FIO) associated with the diffeomorphisms $\kappa_{t}$.

Consider the propagator $U=U(1)$ associated with $\kappa=\kappa_{1}$. $U$ maps a wavepacket microlocalized at $\left(x_{0}, \xi_{0}\right)$ to a wavepacket localized at $\kappa\left(x_{0}, \xi_{0}\right)$. Its action on a quantum observable satisfies a quantum-classical correspondence (called Egorov's theorem in the mathematical literature): for any symbol $a \in S^{0}\left(T^{*} \mathbb{R}^{d}\right)$ of compact support, one has

$$
U^{-1} \mathrm{Op}_{\hbar}(a) U=\mathrm{Op}_{\hbar}(b), \quad b \in S^{0}\left(T^{*} \mathbb{R}^{d}\right), \quad b=a \circ \kappa+\mathcal{O}(\hbar)
$$

More generally, an FIO associated with $\kappa$ will be an operator of the form

$$
\mathcal{M}(\alpha, \hbar)=U \mathrm{Op}_{\hbar}(\alpha)
$$

with $\alpha \in S_{\delta}^{0}\left(T^{*} \mathbb{R}^{d}\right)$ for some $\delta \in[0,1 / 2)$. The FIOs of $\$ 5.2$, in particular the open quantum maps, are of this type. From there one easily shows the "nonunitary" Egorov property (32). Also, the $L^{2}$ norm estimate (34) is obtained from (73). 


\section{REFERENCES}

[1] J. Aguilar and J.M. Combes, A class of analytic perturbations for one-body Schrdinger Hamiltonians, Comm. Math. Phys. 22 (1971) 269-279.

[2] N. Anantharaman, Entropy and the localization of eigenfunctions, Ann. of Math. 168 (2008) 435-475

[3] N. Anantharaman, Spectral deviations for the damped wave equation, GAFA 20 (2010), 593-626.

[4] N.L. Balazs and A. Voros, The quantized baker's transformation, Ann. Phys. (NY) 190 (1989) 1-31

[5] E. B. Bogomolny, Semiclassical quantization of multidimensional systems, Nonlinearity 5 (1992) 805866

[6] E. Bogomolny et al., Trace formula for dielectric cavities. II. Regular, pseudointegrable, and chaotic examples, Phys. Rev. E 83 (2011) 036208

[7] J.-F. Bony and L. Michel, Microlocalization of resonant states and estimates of the residue of the scattering amplitude, Comm. Math. Phys. 246 (2004) 375-402

[8] F. Borgonovi, I. Guarneri and D.L. Shepelyansky, Statistics of quantum lifetimes in a classically chaotic system, Phys. Rev. A 43 (1991) 4517-4520

[9] D. Borthwick, Spectral Theory of Infinite-Area Hyperbolic Surfaces, Birkhauser, Boston, 2007.

[10] R. Bowen and D. Ruelle, The ergodic theory of Axiom A flows, Invent. Math. 29 (1975) 181-202

[11] M. Brin and G. Stuck, Introduction to dynamical systems, Cambridge University Press, 2002

[12] N. Burq, Contrôle de l'équation des plaques en présence d'obstacles strictement convexes, Mémoire de la S.M.F. 55 (1993) 1-126

[13] N. Burq, C. Guillarmou and A. Hassell, Strichartz estimates without loss on manifolds with hyperbolic trapped geodesics, GAFA 20 (2010) 627-656

[14] N. Burq and M. Zworski, Resonance expansions in semiclassical propagation, Commun. Math. Phys. $223(2001) 1-12$

[15] F. Cardoso, G. Popov and G. Vodev, Asymptotics of the number of resonances in the transmission problem, Commun. PDE 26 (2001) 1811-1859

[16] H. Christianson, Semiclassical Non-concentration near Hyperbolic Orbits, J. Funct. Anal. 246 (2007) 145-195; Corrigendum, J. Funct. Anal. 258 (2010) 1060-1065

[17] H. Christianson, Cutoff resolvent estimates and the semilinear Schrödinger equation, Proc. Amer. Math. Soc. 136 (2008) 3513-3520

[18] H. Christianson, Applications of cutoff resolvent estimates to the wave equation, Math. Res. Lett. 16 (2009) $577-590$

[19] Y. Colin de Verdière, Ergodicité et fonctions propres du laplacien, Commun. Math. Phys. 102 (1985) 497-502

[20] K. Datchev, Local smoothing for scattering manifolds with hyperbolic trapped sets, Commun. Math. Phys. 286 (2009) 837-850

[21] K. Datchev and A. Vasy, Gluing semiclassical resolvent estimates, or the importance of being microlocal, preprint 2010, arXiv: 1008:3964

[22] M. Degli Esposti and S. Graffi (eds), The mathematical aspects of quantum maps, Springer, Heidelgerg, 2003.

[23] M. Dimassi and J. Sjöstrand, Spectral Asymptotics in the semiclassical limit, Cambridge University Press, 1999

[24] D. Dolgopyat, On decay of correlations for Anosov flows, Ann. Math. 147 (1998) 357-390

[25] E. Doron and U. Smilansky, Semiclassical quantization of chaotic billiards: a scattering theory approach, Nonlinearity 5 (1992) 1055-1084

[26] A. Eberspächer, J. Main and G. Wunner, Fractal Weyl law for three-dimensional chaotic hard-sphere scattering systems, Phys. Rev. E 82 (2010) 046201 
[27] L.C. Evans and M. Zworski, Lectures on semiclassical analysis, http://math.berkeley.edu/ Zworski/semiclassical.pdf

[28] P. Gaspard and S.A. Rice, Semiclassical quantization of the scattering from a classically chaotic repellor, J. Chem. Phys. 90 (1989), 2242-2254.

[29] T. Gendron, Applications quantiques ouvertes, rapport de stage de Mastère, Institut de Physique Théorique, Saclay, 2009.

[30] C. Gérard, Asymptotique des pôles de la matrice de scattering pour deux obstacles strictement convexes. Mémoires de la SMF Sér. 2, 31 (1988) 1-146

[31] C. Gérard and J. Sjöstrand, Semiclassical resonances generated by a closed trajectory of hyperbolic type, Comm. Math. Phys. 108 (1987) 391-421

[32] C. Guillarmou and F. Naud, Wave decay on convex co-compact hyperbolic manifolds, Commun. Math. Phys. 287 (2009) 489-511

[33] C. Guillarmou and F. Naud, Equidistribution of Eisenstein series in infinite volume, preprint, arXiv: $1107: 2655$

[34] L. Guillopé and M. Zworski, The wave trace for Riemann surfaces, Geom. Funct. Anal. 9 (1999) 11561168

[35] L. Guillopé, K.K. Lin and M. Zworski, The Selberg zeta function for convex co-compact Schottky groups, Commun. Math. Phys. 245 (2004) 149-176

[36] B. Helffer, J. Sjöstrand, Résonances en limite semi-classique, Mém. Soc. Math. France (N.S.) 24/25 (1986) 1-228

[37] M. Ikawa, Decay of solutions of the wave equation in the exterior of several convex bodies, Ann. Inst. Fourier 38 (1988), 113-146

[38] H. Ishio and J.P. Keating, Semiclassical wavefunctions in chaotic scattering systems, J. Phys. A 37 (2004) L217-L223

[39] D. Jakobson and F. Naud, On the resonances of convex co-compact subgroups of arithmetic groups, preprint 2010, arXiv:1011.6264

[40] J.P. Keating, S. Nonnenmacher, M. Novaes and M. Sieber, On the resonance eigenstates of an open quantum baker map, Nonlinearity 21 2591-2624 (2008)

[41] A. Katok and B. Hasselblatt, Introduction to the modern theory of dynamical systems, Cambridge University Press, 1995

[42] J.P. Keating, M. Novaes, M., S. Prado and M. Sieber, Semiclassical structure of chaotic resonance eigenfunctions, Phys. Rev. Lett. 97150406 (2006)

[43] U. Kuhl, R. Höhmann, J. Main and H.-J. Stöckmann, Resonance widths in open microwave cavities studied by harmonic inversion, Phys. Rev. Lett. 100 (2008) 254101

[44] K.K. Lin, Numerical study of quantum resonances in chaotic scattering, J. Comput. Phys. 176 (2002) 295-329

[45] K.K. Lin and M. Zworski, Quantum resonances in chaotic scattering, Chem. Phys. Lett. 355 (2002) 201-205

[46] C. Liverani, On contact Anosov flows, Ann. of Math. 159 (2004) 1275-1312

[47] W. Lu, S. Sridhar and M. Zworski, Fractal Weyl laws for chaotic open systems, Phys. Rev. Lett. 91 (2003) 154101

[48] R.B. Melrose, Polynomial bound on the distribution of poles in scattering by an obstacle, Proc. of Journées EDP 1984, Saint-Jean-de-Monts, Soc. Math. de France, 1984.

[49] F. Naud, Expanding maps on Cantor sets and analytic continuation of zeta functions, Ann. scient. ENS 38 (2005) 116-153

[50] F. Naud, Classical and auantum lifetimes on some non-compact Riemann surfaces, J. Phys. A 38 (2005) 10721-10729 
[51] S. Nonnenmacher, Notes on the minicourse "Entropy of chaotic eigenstates", in Spectrum and Dynamics, D. Jakobson, S. Nonnenmacher and I. Polterovich (Eds.), CRM Proceedings and Lecture Notes 52, AMS, Providence, 2010.

[52] S. Nonnenmacher and M. Rubin, Resonant eigenstates for a quantized chaotic system, Nonlinearity 20 (2007) 1387-1420.

[53] S. Nonnenmacher, J. Sjöstrand and M. Zworski, From open quantum systems to open quantum maps, Commun. Math. Phys. 304 (2011) 1-48

[54] S. Nonnenmacher, J. Sjöstrand and M. Zworski, Fractal Weyl law for open quantum chaotic maps, preprint 2011, arXiv:1105.3128

[55] S. Nonnenmacher and M. Zworski, Distribution of resonances for open quantum maps, Comm. Math. Phys. 269(2007), 311-365.

[56] S. Nonnenmacher and M. Zworski, Fractal Weyl laws in discrete models of chaotic scattering, Journal of Physics A, 38 (2005), 10683-10702.

[57] S. Nonnenmacher and M. Zworski, Quantum decay rates in chaotic scattering, Acta Math. 203 (2009) 149-233.

[58] S. Nonnenmacher and M. Zworski, Semiclassical Resolvent Estimates in Chaotic Scattering, Appl. Math. Res. Expr. 2009 (2009), Article ID abp003

[59] M. Novaes, J.M. Pedrosa, D. Wisniacki, G.G. Carlo and J.P. Keating, Quantum chaotic resonances from short periodic orbits, Phys. Rev. E 80 (2009) 035202(R)

[60] S.J. Patterson, The limit set of a Fuchsian group, Acta Math 136 (1976), 241-273.

[61] S.J. Patterson and P.A. Perry, The divisor of Selberg's zeta function for Kleinan groups, Duke Math. J. 106 (2001) 321-390

[62] P.A. Perry, A Poisson summation formula and lower bounds for resonances in hyperbolic manifolds, IMRN 34 (2003) 1837-1851

[63] V. Petkov and L. Stoyanov, Analytic continuation of the resolvent of the Laplacian and the dynamical zeta function, Ann. PDE 3 (2010) 427-489

[64] M. Pollicott, Some applications of the thermodynamic formalism to manifolds with constant negative curvature, Adv. Math. 85 (1991) 161-192

[65] T. Prosen, General quantum surface-of-section method, J. Phys. A 28 (1995) 4133-4155

[66] J.A. Ramilowski, S.D. Prado, F. Borondo and D. Farrelly, Fractal Weyl law behavior in an open Hamiltonian system, Phys. Rev. E 80 (2009) 055201(R)

[67] M. Saraceno, Classical structures in the quantized baker transformation, Ann. Phys. (NY) 199 (1990) $37-60$

[68] E. Schenck, Energy decay for the damped wave equation under a pressure condition, Commun. Math. Phys. 300 (2010) 375-410

[69] E. Schenck, Exponential stabilization without geometric control, Math. Res. Lett. 18 (2011) 379-388

[70] Schnirelman, A.I., Ergodic properties of eigenfunctions, Usp. Mat. Nauk. 29 (6), 181 (1974).

[71] H. Schomerus and J. Tworzydłlo, Quantum-to-classical crossover of quasi-bound states in open quantum systems, Phys. Rev. Lett. 93 (2004) 154102

[72] D.L.Shepelyansky, Fractal Weyl law for quantum fractal eigenstates, Phys. Rev. E 77 (2008) 015202(R)

[73] S. Shinohara et al., Ray-wave correspondence in limaçon-shaped semiconductor microcavities, Phys. Rev. A 80 (2009) 031801(R)

[74] J. Sjöstrand, Geometric bounds on the density of resonances for semiclassical problems, Duke Math. J., 60 (1990), 1-57

[75] J. Sjöstrand, Asymptotic distribution of eigenfrequencies for damped wave equations, Publ. RIMS 36 (2000) 573-611

[76] J. Sjöstrand and M. Zworski, Complex scaling and the distribution of scattering poles, J. Amer. Math. Soc. 4 (1991) 729-769 
[77] J. Sjöstrand and M. Zworski, Quantum monodromy and semiclassical trace formulae, J. Math. Pure Appl. 81 (2002) 1-33

[78] J. Sjöstrand and M. Zworski, Fractal upper bounds on the density of semiclassical resonances, Duke Math. J. 137 (2007) 381-459

[79] L. Stoyanov, Spectrum of the Ruelle operator and exponential decay of correlations for open billiard flows, Amer. J. Math. 123 (2001) 715-759

[80] L. Stoyanov, Non-integrability of open billiard flows and Dolgopyat type estimates, Ergod. Th. Dynam. Syst., published online, doi:10.1017/S0143385710000933

[81] L. Stoyanov, Spectra of Ruelle transfer operators for Axiom A flows, Nonlinearity 24 (2011) 1089-1120

[82] D. Sullivan, The density at infinity of a discrete group of hyperbolic motions, Publ. Math. IHES 50 (1979) 171-202

[83] S.-H. Tang and M. Zworski, From quasimodes to resonances, Math. Res. Lett. 5 (1998) 261-272

[84] S.-H. Tang and M. Zworski, Resonance expansions of scattered waves, Comm. Pure Appl. Math. 53 (2000) 1305-1334

[85] A. Vasy, Microlocal analysis of asymptotically hyperbolic and Kerr-de Sitter spaces, with an appendix by S. Dyatlov, preprint 2010 arXiv:1012.4391

[86] G. Vodev, Sharp upper bounds on the number of scattering poles in even-dimensional spaces, Duke Math. J. 74 (1994) 1-17

[87] T. Weich, Resonances in open expanding maps and in an experimental realization of the three disk system, Diplomarbeit, Universität Marburg, 2010

[88] J. Wiersig and J. Main, Fractal Weyl law for chaotic microcavities: Fresnel's laws imply multifractal scattering, Phys. Rev. E 77 (2008) 036205

[89] A. Wirzba, Quantum mechanics and semiclassics of hyperbolic n-disk scattering systems, Phys. Rep. 309 (1999) 1-116

[90] Zelditch, S. Uniform distribution of eigenfunctions on compact hyperbolic surfaces, Duke Math. J. 55 (1987) 919-941

[91] M. Zworski, Sharp polynomial bounds on the number of scattering poles, Duke Math. J. 59 (1989) $311-323$

[92] M. Zworski, Dimension of the limit set and the distribution of resonances for convex co-compact hyperbolic surfaces, Invent. Math. 136 (1999) 353-409

Institut de Physique ThÉorique, CEA/DSM/IPhT, Unité de Recherche associée au CNRS, CEA/Saclay, 91191 Gif-Sur-Yvette, France

E-mail address: snonnenmacher@cea.fr 


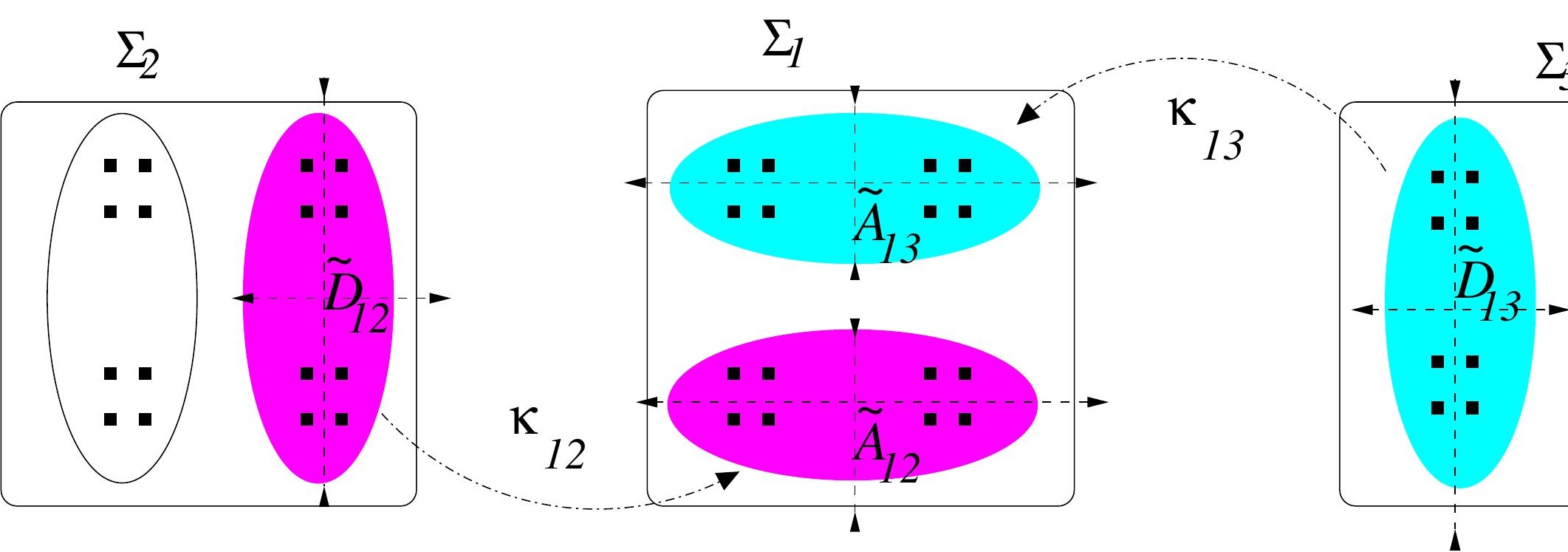

